\title{
CHANDRA STUDY OF THE CEPHEUS B STAR-FORMING REGION: STELLAR POPULATIONS AND THE INITIAL MASS FUNCTION
}

\author{
Konstantin V. Getman, ${ }^{1}$ Eric D. Feigelson, ${ }^{1}$ Leisa Townsley, ${ }^{1}$ Patrick Broos, ${ }^{1}$ \\ Gordon Garmire, ${ }^{1}$ and Masahiro Tsujimoto ${ }^{1,2}$ \\ Received 2005 November 2; accepted 2006 January 18
}

\begin{abstract}
The Cepheus B (Cep B) molecular cloud and a portion of the nearby Cep OB3b OB association, one of the most active regions of star formation within $1 \mathrm{kpc}$, have been observed with the ACIS detector on board the Chandra $X$-Ray Observatory. We detect $431 \mathrm{X}$-ray sources, of which $89 \%$ are confidently identified as clustered pre-mainsequence (PMS) stars. Two main results are obtained. First, we provide the best census to date for the stellar population of the region. We identify many members of two rich stellar clusters: the lightly obscured Cep OB3b association and the deeply embedded cluster in Cep B, whose existence was previously traced only by a handful of radio sources and T Tauri stars. Second, we find a discrepancy between the X-ray luminosity functions of the Cep OB3b and the Orion Nebula cluster. This may be due to the different initial mass functions of the two regions (an excess of $\simeq 0.3 M_{\odot}$. stars) or different age distributions. Several other results are obtained. A diffuse X-ray component seen in the field is attributed to the integrated emission of unresolved low-mass PMS stars. The X-ray emission from HD $217086(\mathrm{O} 7 \mathrm{n})$, the principle ionizing source of the region, follows the standard model, involving many small shocks in an unmagnetized radiatively accelerated wind. X-ray source 294 joins a number of similar superflare PMS stars for which long magnetic structures may connect the protoplanetary disk to the stellar surface.
\end{abstract}

Subject headings: ISM: individual (Cepheus molecular cloud) - open clusters and associations: individual (Cepheus OB3) - stars: formation - stars: luminosity function, mass function - stars: pre-main-sequence $-\mathrm{X}$-rays: stars

Online material: machine-readable tables

\section{INTRODUCTION}

Cepheus B (Cep B), the hottest CO component of the Cepheus molecular cloud (Sargent 1977, 1979), is located near the northwestern edge of the cloud at a distance of $725 \mathrm{pc}$ from the Sun (Blaauw et al. 1959; Crawford \& Barnes 1970). Outside Cep B, to the north and west of the cloud, lies the stellar Cep OB3 association, composed of two subgroups of stars of different ages with the youngest (Cep OB3b) lying closer to the molecular cloud (Blaauw 1964; Jordi et al. 1996; see our Fig. 1).

Sargent (1979) considered Cep OB3 to be a good example of sequential star formation, following the model of Elmegreen \& Lada (1977). The interface between the molecular cloud and the OB star association is clearly delineated by the optically visible $\mathrm{H}$ II region Sharpless 155 (S155), where cloud material is ionized and heated by the radiation field of the youngest generation of the Cep OB association (Panagia \& Thum 1981). The photodissociation region (PDR) at S155 is favorably oriented to reveal the progression of star formation. As the surface of the cloud is being eroded by the early-type stars, the cloud edge moves eastward across the observer's field of view, with new stars emerging from the obscuring molecular cloud. Radio continuum (Felli et al. 1978; Testi et al. 1995), far-infrared, CO (Minchin et al. 1992), and near-infrared (NIR) studies (Moreno-Corral et al. 1993; Testi et al. 1995) reveal a few young stars embedded in the Cep B cloud behind the PDR. Sources located on the edge of the molecular cloud and exhibiting high extinction were suggested to represent a third generation of star formation triggered by the $\mathrm{H}$ II region shock propagating into the cloud.

\footnotetext{
${ }^{1}$ Department of Astronomy and Astrophysics, 525 Davey Laboratory, Pennsylvania State University, University Park, PA 16802.

2 Department of Physics, Rikkyo University, 3-34-1, Nishi-Ikebukuro, Toshima, Tokyo 171-8501, Japan.
}

The low-mass pre-main-sequence (PMS) stellar population of Cep OB3b can be effectively discriminated using X-ray emission due to the heavy contamination in optical and NIR surveys by both foreground and background Galactic field stars. X-ray surveys are complementary to optical and NIR surveys because they trace magnetic activity (mainly plasma heated in violent magnetic reconnection flares) rather than photospheric or circumstellar disk blackbody emission, and PMS X-ray emission is elevated $10^{1}-10^{4}$ above main-sequence (MS) levels (Preibisch et al. 2005). A ROSAT (Röntgensatellit) X-ray study of Cep OB3b identified over 50 likely PMS stars with $A_{V}<3$ mag and $L_{\mathrm{X}} \gtrsim$ $10^{30} \mathrm{ergs} \mathrm{s}^{-1}$ (Naylor \& Fabian 1999). Ten of these ROSAT sources are coincident with optical sources, and four of these are spectroscopically confirmed classical and weak-lined T Tauri stars ( Pozzo et al. 2003).

Much more can be achieved with the current generation of X-ray telescopes, particularly the Chandra X-Ray Observatory, with its excellent high-resolution mirrors and low-noise detectors. Studies of the Orion Nebula region demonstrate that Chandra images can penetrate up to $A_{V} \simeq 500$ mag into the cloud (Getman et al. 2005b; Grosso et al. 2005), considerably deeper than NIR surveys in the JHKL bands. Chandra is also effective in resolving crowded fields down to $\simeq 0^{\prime \prime} 7$ scales (Getman et al. 2005a). In $\mathrm{X}$-rays, OB stars are often not much brighter than PMS stars, so multiple systems associated with OB stars can be identified (Stelzer et al. 2005). Chandra X-ray studies are particularly effective in uncovering heavily obscured low-mass cloud populations and in discriminating PMS populations from unrelated older stars.

We present here a Chandra study of the region using the Advanced CCD Imaging Spectrometer (ACIS) detector. Over 380 PMS stars in two rich clusters are found, concentrated in the lightly obscured Cep OB3b cluster and a previously unknown embedded cluster, and we infer the existence of an additional $700-800$ 


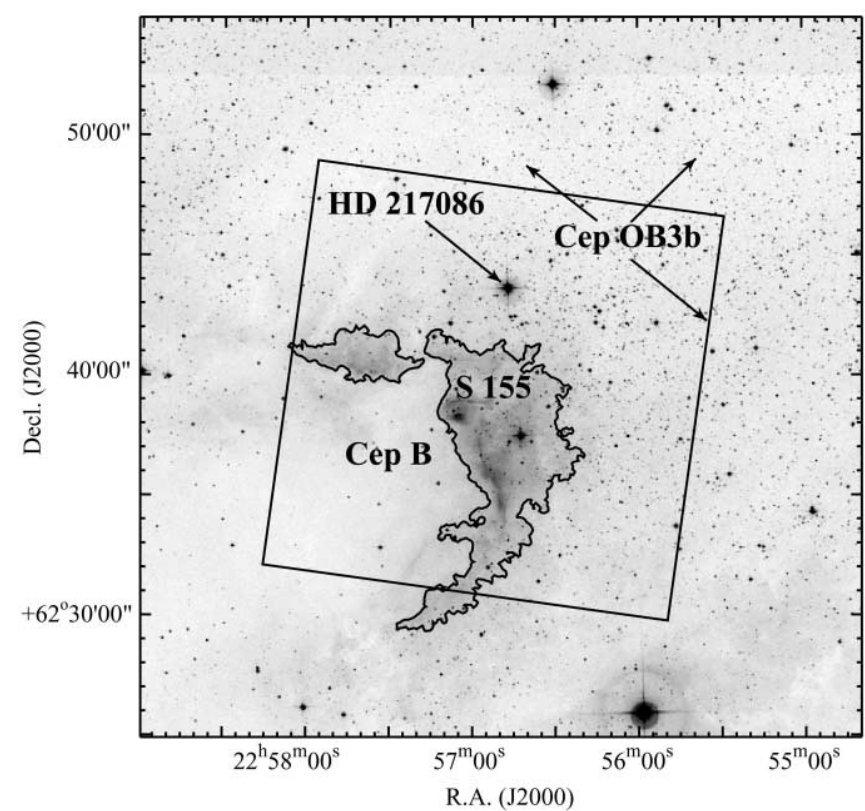

FIG. 1. $-R$-band image covering $0.5 \times 0.5$ of the Cep B and Cep OB3b neighborhood from the Digitized Sky Survey (DSS). The Chandra $17^{\prime} \times 17^{\prime}$ ACIS-I field is outlined by the square. Cep B, the hottest component of the Cepheus molecular cloud, is at the bottom left corner of the Chandra field. To the north and west lies Cep OB3b, the youngest of two subgroups of the stellar Cep OB3 association. The interface between Cep B and Cep OB3 is delineated by the $\mathrm{H}$ II region, S155. The most massive and optically bright star in our field, HD 217086 (O7n) is labeled. North is up; east is to the left.

low-mass members. Only $\simeq 7 \%-9 \%$ of the detected X-ray sources are attributable to contaminating Galactic and extragalactic populations. The observation and source lists are described in $\S 2$. Identification with 2MASS ( Two Micron All Sky Survey) counterparts and their NIR properties are considered in $\S 3$. X-ray fluxes and their errors are determined in $\S 4$. X-ray sources unrelated to the cloud are discussed in $\S 5$. The discovery of two rich clusters is described in $\S 6$. Their stellar populations are discussed in $\S 7$ using measured X-ray luminosity functions (XLFs) and initial mass functions (IMFs). The origin of the soft diffuse X-ray emission discovered here is discussed in $\S 8$. Finally, we address two individual stars of particular interest: HD 217086, the most massive star in Cep OB3b ( $\S 9.1)$, and Chandra source 294, which exhibited an unusually luminous and hot X-ray flare (§ 9.2).

\section{THE CHANDRA OBSERVATION AND SOURCE LIST}

\subsection{Observation and Data Reduction}

The observation of Cep B and Cep OB3b was obtained on 2003 March 11.51-11.88 with the ACIS camera (Garmire et al. 2003) on board Chandra (Weisskopf et al. 2002). We consider here only results arising from the imaging array (ACIS-I) of four abutted $1024 \times 1024$ pixel front-side-illuminated charge-coupled devices (CCDs) covering about $17^{\prime} \times 17^{\prime}$ on the sky. The S2 and $\mathrm{S} 3$ detectors in the spectroscopic array (ACIS-S) were also operational, but as the telescope point-spread function (PSF) is considerably degraded far off-axis, the S2-S3 data are omitted from the analysis. The aim point of the array is $22^{\mathrm{h}} 56^{\mathrm{m}} 49^{\mathrm{s}} .4$, $+62^{\circ} 39^{\prime} 55^{\prime \prime}$. 6 (J2000.0), and the satellite roll angle (i.e., orientation of the CCD array relative to the north-south direction) is $7^{\circ} .9$. The total net exposure time of our observation is $30 \mathrm{ks}$, with no background flaring or data losses.

Data reduction, source detection, and extraction have been done using procedures similar to those described in detail by
Getman et al. (2005b). Briefly, the data were partially corrected for CCD charge transfer inefficiency (Townsley et al. 2002), cleaned with "grade," "status," and "good-time interval" filters, trimmed of background events outside of the $0.5-8.0 \mathrm{keV}$ band, and cleaned of bad pixel columns with energies of $<0.7 \mathrm{keV}$ left by the standard processing (see Appendix B of Townsley et al. 2003 for more details). Based on several dozen matches between bright Chandra and 2MASS point sources, a correction to the absolute astrometry was applied and the slight PSF broadening from the Chandra X-Ray Center's software randomization of positions was removed. Source searching was performed with data image and exposure maps constructed at three spatial resolutions $\left(0.5,1\right.$." 0 , and 1.4 pixel $\left.^{-1}\right)$ using the CIAO wavdetect tool. Those procedures were performed with CIAO software package 3.1, LHEASOFT 5.3, the Penn State CTI corrector version 1.42, and the ACISExtract (AE) package version 3.67. The latter two tools were developed at Penn State. ${ }^{3}$

\subsection{Source Detection and Characterization}

Figure 2 shows the resulting smoothed image of the ACIS-I field. More than 400 point sources are seen. ${ }^{4}$ A wavelet-based source detection procedure (wavdetect) is applied. It is followed by visual examination to locate other candidate sources, mainly close doubles and sources near the detection threshold. Using AE, photons are extracted from the polygonal contours of $\sim 90 \%$ encircled energy using detailed position-dependent models of the PSF. Background is measured locally in source-free regions. The list of candidate sources is trimmed to omit sources with fewer than 3 background-subtracted counts in the PSF (i.e., NetFull/PSF $<3$; these and other quantities are defined in the notes of Table 1). This resulted in a catalog of 431 sources, which are listed in Table 1. Twenty-seven of the 31 weakest sources with 3 counts $<$ NetFull/PSF $<5$ counts (median source significance $=1.1$, which corresponds [Getman et al. 2005b] to the $2.2 \sigma$ detection threshold of PWDetect) have NIR counterparts ( 3 ); we thus believe most of these are real despite their marginal statistical significance. One hundred and eight sources with $5<$ NetFull $/ \mathrm{PSF}<10$ have median significance $=1.8$ (3.6 $\sigma$ detection threshold of PWDetect).

The AE package provides a variety of source characteristics, including celestial position, off-axis angle, net and background counts within the PSF-based extraction area, source significance assuming Poisson statistics, effective exposure (corrected for telescope vignetting, satellite dithering, and bad CCD pixels), median energy after background subtraction, a variability indicator extracted from the one-sided Kolmogorov-Smirnov statistics, and occasional anomalies related to chip gap or field edge positions. These are all reported in Table 1; see Getman et al. (2005b) for a fuller description of these quantities.

\section{2MASS COUNTERPARTS AND THEIR PROPERTIES}

\subsection{Identification with Cataloged 2MASS Sources}

An automated cross-correlation between the Chandra source positions and 2MASS NIR catalogs was made using a search radius of $1^{\prime \prime}$ within $\simeq 3$. 5 of the ACIS field center, and a search radius of $2^{\prime \prime}$ in the outer regions of the field where the Chandra PSF deteriorates. To evaluate the merits of this automated procedure, we performed a careful visual examination of each source.

\footnotetext{
3 Descriptions and codes for CTI correction and ACIS Extract can be found at http://www.astro.psu.edu/users/townsley/cti and http://www.astro.psu.edu/xray/ docs/TARA/ae_users_guide.html, respectively.

${ }^{4}$ We do not show here the raw image of the field as it gives a misleading impression of high background and low resolution.
} 


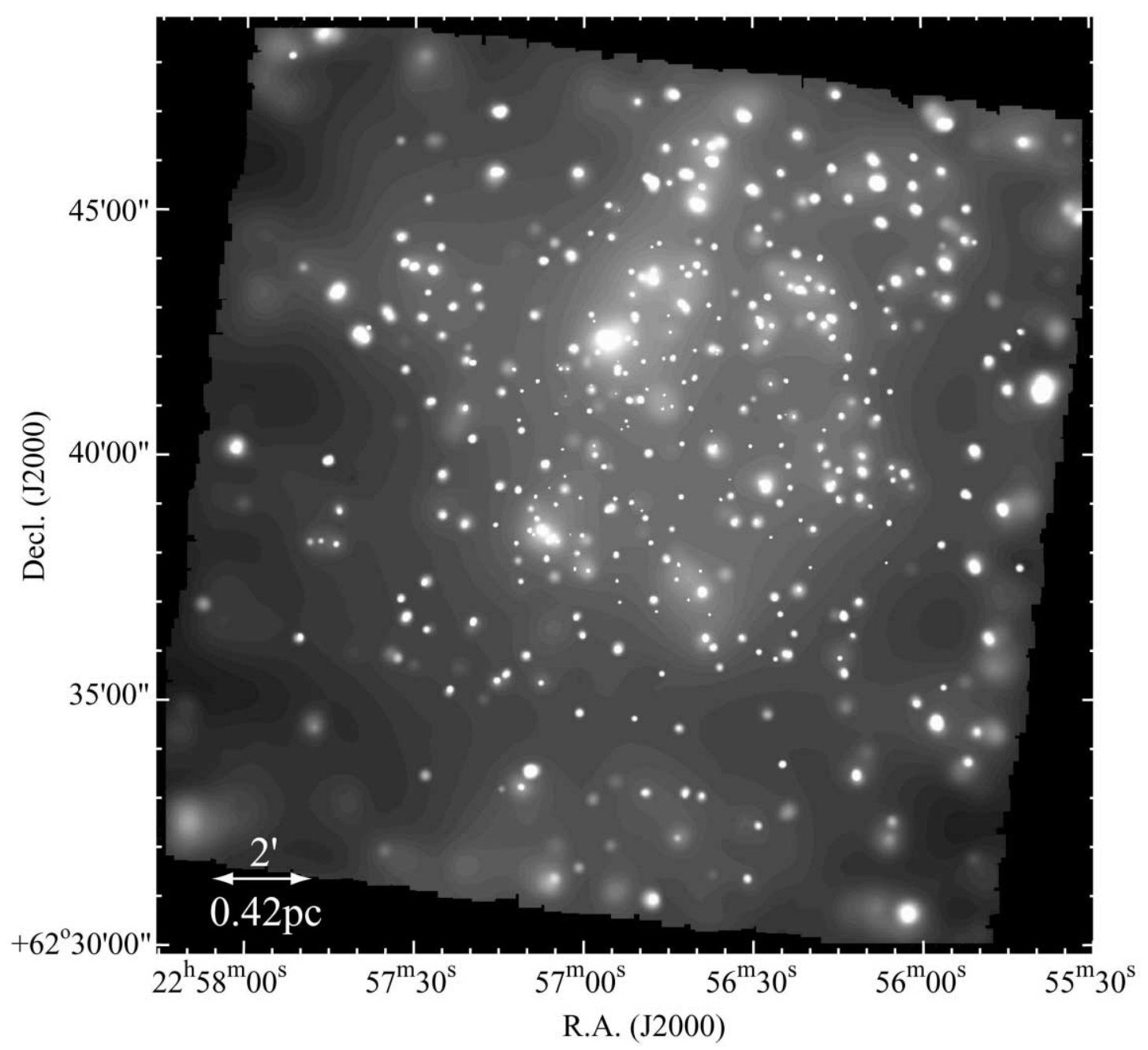

Fig. 2.-Adaptively smoothed Cep B ACIS-I image in the full $0.5-8.0 \mathrm{keV}$ band. Smoothing has been performed at the $2.5 \sigma$ level, and gray scales are logarithmic.

In $99 \%$ of the cases, the identifications are clear and unambiguous. Seventeen matches near the edges of the field with Chandra2MASS separations greater than $2^{\prime \prime}$ were added to the catalog. The result is that 385 out of 431 (89\%) Chandra sources have NIR counterparts in the 2MASS catalog (Table 2).

After a correction to the Chandra boresight of $\Delta$ R.A. $=$ +0 " 13 and $\Delta$ decl. $=-0$ " 14 , mentioned in $\S 2.1$, to bring it into the 2MASS/Hipparcos reference frame, the median Chandra2MASS separations are better than 0.21 at the central part of the field (off-axis angle $<3.5$ ) and 0".47 at larger off-axis angles. This excellent astrometric agreement between 2MASS and Chandra positions is similar to that achieved in the Orion Nebula cluster (ONC) field (Getman et al. 2005b).

\subsection{X-Ray Sources with Uncataloged 2MASS Counterparts}

Of the 46 X-ray sources not associated with 2MASS cataloged stars, 14 are soft (MedE $<2 \mathrm{keV}$ ) and 32 are hard (MedE $>2 \mathrm{keV})$. Visual examination of the 2MASS atlases shows that $12 \mathrm{soft}$ (sources 78, 103, 170, 222, 242, 243, 249, 296, 309, 340, 343, and 374) and two hard (sources 395 and 403) X-ray sources without identification lie very close to bright NIR stars. As an example, expanded views of the 2MASS atlas around $12 \mathrm{soft}$ X-ray sources are shown in Figure 3. The nearby cataloged 2MASS stars are often flagged as an extended NIR source. In the case of sources 242 and 243, Chandra found two uncataloged compan- ions to a bright 2MASS star. X-ray source 340 is suspiciously close to an unregistered bright 2MASS source but does not coincide with it; this may be emission from a Herbig-Haro shock, but the lack of optical data does not allow us to speculate on it. The only two soft X-ray sources that are truly not seen in the NIR 2MASS are sources 26 and 214. These two are the hardest among the soft sample and perhaps are too absorbed to be detected in the NIR. Later in the membership study $(\S 5)$ we consider these 14 soft sources, including sources 26,214 , and 340, and two hard sources, 395 and 403, as likely members of the Cepheus populations.

\subsection{NIR Properties of X-Ray-detected Sources}

Figures 4 and 5 compare NIR properties of all cataloged 2MASS sources in the ACIS-I field with the 385 detected in X-rays (not including uncataloged 2MASS sources from $\S 3.2$ ). The latter sources are clustered in NIR color space at $A_{V} \sim 2-$ 4 mag with only $\sim 10$ having NIR colors consistent with foreground MS stars. NIR colors for $\sim 30,8 \%$ of the X-ray-selected population, show $K$-band excesses that indicate the presence of dusty protoplanetary disks.

Pozzo et al. (2003) found an age of $\sim 1$ Myr for four out of six PMS candidates within their $1 \mathrm{a}$ and $2 \mathrm{~b}$ fields (see their Fig. 15), which partially overlap our ACIS-I field. In Figure 5 we show the 1 Myr isochrone in the $J$ versus $J-H$ color-magnitude diagram 
TABLE 1

Basic Source Properties

\begin{tabular}{|c|c|c|c|c|c|c|c|c|c|c|c|c|c|c|}
\hline & & & TION (J2000.C & & & & & & & & & ACTERISTICS & & \\
\hline & SOURCE & & & & & & EXTRACTED C & UNTS & & & & & & \\
\hline $\begin{array}{l}\text { Seq. No. } \\
\text { (1) }\end{array}$ & $\begin{array}{c}\text { CXOCEPB } \\
\text { (2) }\end{array}$ & $\begin{array}{l}\text { (deg) } \\
\text { (3) }\end{array}$ & $\begin{array}{l}\text { (deg) } \\
\text { (4) }\end{array}$ & $\begin{array}{c}(\operatorname{arcmin}) \\
(5)\end{array}$ & $\begin{array}{l}\text { NetFull } \\
\text { (6) }\end{array}$ & $\begin{array}{l}\Delta \text { NetFull } \\
\text { (7) }\end{array}$ & $\begin{array}{l}\text { BkgdFull } \\
(8)\end{array}$ & $\begin{array}{l}\text { NetHard } \\
\text { (9) }\end{array}$ & $\begin{array}{l}\text { PSF Fraction } \\
\text { (10) }\end{array}$ & $\begin{array}{l}(\sigma) \\
(11)\end{array}$ & $\begin{array}{l}\text { Anomaly }^{\mathrm{a}} \\
\text { (12) }\end{array}$ & $\begin{array}{l}\text { Variability } \\
\text { (13) }\end{array}$ & $\begin{array}{l}(\mathrm{ks}) \\
(14)\end{array}$ & $\begin{array}{c}(\mathrm{keV}) \\
(15)\end{array}$ \\
\hline $1 \ldots \ldots \ldots \ldots \ldots \ldots$ & $225531.25+624451.7$ & 343.880235 & 62.747708 & 10.22 & 10.2 & 4.0 & 1.8 & 2.9 & 0.70 & 2.2 & ge - - & $\ldots$ & 11.7 & 1.70 \\
\hline $2 \ldots \ldots \ldots \ldots \ldots \ldots \ldots$ & $225533.30+624457.2$ & 343.888775 & 62.749237 & 10.06 & 9.1 & 4.9 & 8.9 & 0.0 & 0.90 & 1.7 & $\ldots$ & $\mathrm{a}$ & 25.2 & 1.16 \\
\hline $3 \ldots \ldots$ & $225535.75+624235.4$ & 343.898960 & 62.709839 & 8.85 & 8.0 & 3.9 & 3.0 & 0.2 & 0.90 & 1.8 & g - - - & $\ldots$ & 21.2 & 1.26 \\
\hline $4 \ldots \ldots \ldots \ldots \ldots \ldots \ldots$ & $225537.53+624128.1$ & 343.906375 & 62.691159 & 8.38 & 101.7 & 10.8 & 3.3 & 67.9 & 0.89 & 9.0 & - - - & $\mathrm{b}$ & 26.3 & 2.65 \\
\hline $5 \ldots \ldots \ldots \ldots \ldots \ldots$ & $225538.33+624117.8$ & 343.909731 & 62.688296 & 8.26 & 542.3 & 23.9 & 3.7 & 178.4 & 0.89 & 22.2 & $-\cdots$ & $\mathrm{c}$ & 26.6 & 1.64 \\
\hline 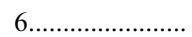 & $225540.99+624625.9$ & 343.920829 & 62.773882 & 10.18 & 27.6 & 6.7 & 9.4 & 15.2 & 0.90 & 3.8 & $\ldots$ & $\mathrm{a}$ & 25.3 & 2.65 \\
\hline 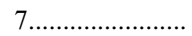 & $225541.56+623905.9$ & 343.923167 & 62.651662 & 7.83 & 6.4 & 3.5 & 2.6 & 0.0 & 0.90 & 1.5 & $\ldots$ & $\mathrm{a}$ & 27.0 & 1.01 \\
\hline 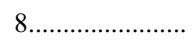 & $225541.74+624232.1$ & 343.923948 & 62.708936 & 8.18 & 17.1 & 5.0 & 2.9 & 2.3 & 0.90 & 3.1 & $\ldots$ & $\mathrm{a}$ & 24.5 & 1.45 \\
\hline 9 & $225542.16+623742.3$ & 343.925698 & 62.628442 & 8.03 & 6.6 & 3.7 & 3.4 & 0.0 & 0.90 & 1.5 & - - - & $\mathrm{a}$ & 24.9 & 1.20 \\
\hline $10 \ldots \ldots \ldots \ldots \ldots$ & $225544.19+624122.1$ & 343.934156 & 62.689499 & 7.61 & 29.4 & 6.2 & 2.6 & 8.4 & 0.89 & 4.4 & $\ldots$ & $\mathrm{a}$ & 26.9 & 1.38 \\
\hline $11 \ldots \ldots \ldots \ldots \ldots \ldots$ & $225544.21+624213.4$ & 343.934247 & 62.703740 & 7.82 & 19.0 & 5.1 & 2.0 & 2.7 & 0.89 & 3.3 & $\ldots$ & $\mathrm{a}$ & 26.8 & 1.20 \\
\hline $12 \ldots \ldots \ldots \ldots \ldots \ldots \ldots$ & $225545.08+623854.6$ & 343.937848 & 62.648524 & 7.45 & 69.6 & 9.0 & 2.4 & 20.4 & 0.89 & 7.3 & $\ldots$ & $\mathrm{c}$ & 27.2 & 1.48 \\
\hline $13 \ldots \ldots \ldots \ldots \ldots$ & $225547.50+624157.6$ & 343.947954 & 62.699356 & 7.38 & 28.1 & 6.0 & 1.9 & 0.8 & 0.89 & 4.3 & $\ldots$ & $\mathrm{a}$ & 27.0 & 1.34 \\
\hline $14 \ldots \ldots \ldots \ldots \ldots \ldots \ldots$ & $225547.59+623614.8$ & 343.948310 & 62.604131 & 7.99 & 38.6 & 7.0 & 3.4 & 9.2 & 0.90 & 5.1 & g - - - & $\ldots$ & 26.2 & 1.23 \\
\hline $15 \ldots \ldots \ldots \ldots \ldots \ldots \ldots$ & $225549.82+623421.9$ & 343.957606 & 62.572759 & 8.82 & 15.2 & 4.9 & 3.8 & 12.5 & 0.90 & 2.8 & $\ldots$ & $\mathrm{a}$ & 26.8 & 2.99 \\
\hline
\end{tabular}

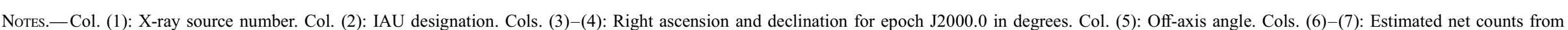

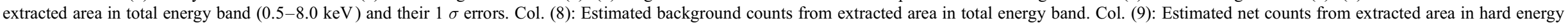

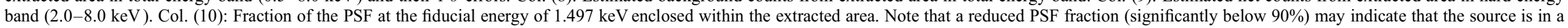

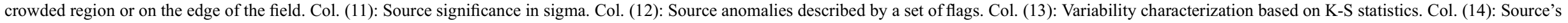

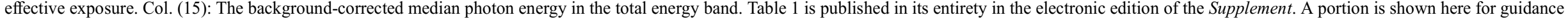
regarding its form and content.

Source anomalies: $\mathrm{g}=$ fractional time that source was on a detector (FRACEXPO from mkarf) is $<0.9$; $\mathrm{e}=$ source on field edge; $\mathrm{p}=$ source piled up; $\mathrm{s}=$ source on readout streak

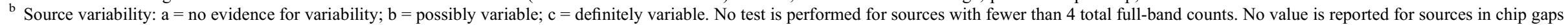
or on field edges. 
TABLE 2

2MASS COUNTERPARTS

\begin{tabular}{|c|c|c|c|c|c|c|c|c|c|c|c|}
\hline $\begin{array}{l}\text { Seq. No. } \\
\text { (1) }\end{array}$ & $\begin{array}{l}\text { CXOCEPB } \\
\text { (2) }\end{array}$ & $\begin{array}{l}\text { 2MASS } \\
\text { (3) }\end{array}$ & $\begin{array}{l}\text { Offset } \\
(\operatorname{arcsec}) \\
(4)\end{array}$ & $\begin{array}{c}J \\
(\mathrm{mag}) \\
(5)\end{array}$ & $\begin{array}{c}H \\
(\mathrm{mag}) \\
(6)\end{array}$ & $\begin{array}{c}K_{s} \\
(\mathrm{mag}) \\
(7)\end{array}$ & $\begin{array}{c}\text { Photom. Flag }{ }^{\mathrm{a}} \\
\text { (8) }\end{array}$ & $\begin{array}{l}\text { Conf. }{ }^{\text {b Flag }} \\
\text { (9) }\end{array}$ & $\begin{array}{c}A_{V, \mathrm{IR}} \\
(\mathrm{mag}) \\
(10)\end{array}$ & $\begin{array}{c}M_{\mathrm{IR}} \\
\left(M_{\odot}\right) \\
(11)\end{array}$ & $\begin{array}{c}\log L_{\text {bol, IR }} \\
\left(L_{\odot}\right) \\
(12)\end{array}$ \\
\hline $2 \ldots \ldots \ldots \ldots \ldots$ & $225533.30+624457.2$ & $22553312+6244596$ & 2.72 & 13.93 & 13.00 & 12.69 & AAA & 000 & 1.39 & 0.27 & -0.57 \\
\hline $3 \ldots \ldots \ldots \ldots \ldots$ & $225535.75+624235.4$ & $22553545+6242343$ & 2.29 & 12.54 & 11.67 & 11.38 & AEE & 000 & 0.46 & 0.68 & -0.10 \\
\hline $4 \ldots \ldots \ldots \ldots \ldots$ & $225537.53+624128.1$ & $22553745+6241280$ & 0.57 & 12.57 & 11.25 & 10.47 & AAA & 000 & 6.15 & 1.81 & 0.74 \\
\hline $5 \ldots \ldots \ldots \ldots \ldots$ & $225538.33+624117.8$ & $22553834+6241177$ & 0.14 & 12.99 & 11.96 & 11.44 & AAA & 000 & 1.62 & 0.63 & -0.14 \\
\hline $8 \ldots \ldots \ldots \ldots \ldots$ & $225541.74+624232.1$ & $22554177+6242322$ & 0.19 & 14.11 & 13.17 & 12.72 & AAA & $\mathrm{c} 00$ & 1.49 & 0.24 & -0.63 \\
\hline $9 \ldots \ldots \ldots \ldots \ldots$ & $225542.16+623742.3$ & $22554205+6237426$ & 0.84 & 15.28 & 14.32 & 14.01 & AAA & 000 & 2.47 & 0.12 & -1.01 \\
\hline $10 \ldots \ldots \ldots \ldots$ & $225544.19+624122.1$ & $22554423+6241220$ & 0.29 & 14.35 & 13.45 & 13.15 & AAA & 000 & 1.51 & 0.18 & -0.74 \\
\hline $11 \ldots \ldots \ldots \ldots$ & $225544.21+624213.4$ & $22554407+6242130$ & 1.05 & 13.94 & 13.04 & 12.80 & AAA & 000 & 1.14 & 0.25 & -0.61 \\
\hline $12 \ldots \ldots \ldots \ldots$ & $225545.08+623854.6$ & $22554507+6238547$ & 0.07 & 14.05 & 13.13 & 12.86 & AAA & 000 & 1.45 & 0.24 & -0.62 \\
\hline $13 \ldots \ldots \ldots \ldots$ & $225547.50+624157.6$ & $22554749+6241576$ & 0.12 & 12.96 & 12.05 & 11.78 & AAA & 000 & 0.70 & 0.53 & -0.25 \\
\hline
\end{tabular}

Notes.-Col. (1): X-ray source number. Col. (2): IAU designation. Col. (3): 2MASS source. Col. (4): Chandra-2MASS positional offset. Cols. (5)-(7): 2MASS $J H K_{s}$ magnitudes. Cols. (8)-(9): 2MASS photometry quality and confusion and contamination flags. Cols. (10)-(12): Visual absorption, stellar mass, and bolometric luminosity derived from color-magnitude diagram by dereddening 2MASS photometric colors to the 1 Myr PMS isochrone. Table 2 is published in its entirety in the electronic edition of the Supplement. A portion is shown here for guidance regarding its form and content.

${ }^{a}$ Photometry quality flag with three characters referring to the $J H K_{s}$ bands: $\mathrm{A}=$ very high significance detection $(>10 \mathrm{~S} / \mathrm{N})$; B $=$ high significance detection $(>7 \mathrm{~S} / \mathrm{N})$; $\mathrm{C}=$ moderate significance detection $(>5 \mathrm{~S} / \mathrm{N}) ; \mathrm{D}=$ low significance detection; $\mathrm{E}=$ point-spread fitting poor $; \mathrm{F}=$ reliable photometric errors not available; $\mathrm{U}=$ upper limit on magnitude (source not detected); $\mathrm{X}=$ source is detected but no valid brightness estimate is available.

b 2MASS confusion and contamination flag with three characters referring to the $J H K_{s}$ bands: $0=$ source unaffected by artifacts; $\mathrm{b}=$ band-merge confusion ( possible multiple source); $\mathrm{c}=$ photometric confusion from nearby star; $\mathrm{d}=$ diffraction spike confusion from nearby star; $\mathrm{p}=$ persistence contamination from nearby star; $\mathrm{s}=$ electronic stripe from nearby star.

with reddening loci for PMS stars of different masses (see footnote 6 below for references). We compare locations of our X-ray sources on the color-magnitude diagram with the 1 Myr PMS isochrone and estimate their masses, bolometric luminosities, and local absorptions. Most of the X-ray sources are consistent with PMS stars with masses of 0.1-1 $M_{\odot}$ at a distance of $725 \mathrm{pc}$ (Testi et al. 1995) subject to $1 \mathrm{mag}<A_{V}<3 \mathrm{mag}$ absorption. Several dozen appear to be more obscured PMS stars with $5<A_{V}<15$.

Table 2 provides information on the 385 2MASS counterparts, including their offsets from Chandra positions, photometric magnitudes, photometry quality, and confusion flags. With the assumption that most of the detected X-ray sources are 1 Myr old PMS stars, we also give our estimates of stellar masses, absorptions, and bolometric luminosities based on the NIR color-magnitude diagram in Figure 5.

\subsection{Understanding the Background Stellar Population}

We examined Monte Carlo simulations of the Galactic stellar population expected in the direction of our ACIS field $(l, b)=$ $\left(110^{\circ} 2,+2^{\circ} .7\right)$ based on the stellar population synthesis model of Robin et al. (2003). ${ }^{5}$ Within the ACIS field of view, the Besançon model predicts $\sim 100-150$ foreground $(d<725 \mathrm{pc})$ and $\sim 1400$ 3100 background MS stars, giants, and subgiants within the 2MASS sensitivity limit around $J<16-17 \mathrm{mag}$.

The simulations help us to understand the bimodal distribution of 2MASS stars in the color-magnitude diagram (this was

\footnotetext{
5 This was computed by the Web service provided by the Robin et al. (2003) group at Besançon at http:// bison.obs-besancon.fr/modele. In addition to the smooth absorption component provided in the model, we added a cloud at $d=725 \mathrm{pc}$ with $A_{V} \sim 2.5 \mathrm{mag}$, as inferred from the average NIR colors of Chandra sources shown in Figs. 4-5. As we have not considered the patchy denser molecular cloud that covers about one-fourth of the ACIS-I field of view, the inferred background population may be somewhat overestimated. We refer to this hereafter as the Besançon model.
}

previously noticed from $V$ vs. $V-I$ optical diagrams by Pozzo et al. [2003]). The model indicates that due to its sensitivity limits, 2MASS will preferentially detect the M, K, and G MS stars at distances of $\sim 0.8-3.6 \mathrm{kpc}$, but will detect large populations of A and F MS stars, and subgiants and giants of all spectral types, located at distances of $\sim 5.4-5.7 \mathrm{kpc}$. Loci for these subpopulations are shown in the NIR color-magnitude diagram of Figure $5 .{ }^{6}$ The region occupied by most of our X-ray sources, PMS stars with $A_{V} \sim 2-4 \mathrm{mag}$, is also the characteristic location for $\sim 400-500$ older $(>1 \mathrm{Gyr})$ background giants. None of those giants is expected to emit X-rays detectable in our observation ( $(5.3)$.

\section{X-RAY SPECTROSCOPY AND FLUXES}

The XLF of stars in young stellar clusters exhibits a range of $10^{5}$ in X-ray luminosity (Feigelson et al. 2005). In relatively short exposures of distant clusters, the majority of sources have

\footnotetext{
${ }^{6}$ We give here details concerning the $J$ vs. $J-H$ color magnitude diagram in Fig. 5. Black dots show Chandra sources with 2MASS counterparts, and gray dots show all 2MASS sources in the field. The ZAMS (thick black dotted line) is obtained from Tables 7.6 and 15.7 of Cox (2000). The 1 Myr isochrone (thick black solid line) is from Baraffe et al. (1998) for $0.02 M_{\odot} \leq M \leq 1.4 M_{\odot}$ and Siess et al. (2000) for $1.4 M_{\odot} \leq M \leq 7.0 M_{\odot}$. Reddening vectors of $A_{V} \sim 10 \mathrm{mag}$ (dashed lines) are given for $0.075,0.1,0.2,0.5,1.0,2.0$, and $4.0 M_{\odot}$. Overlaid on this color-magnitude diagram are outlines of the distributions of expected foreground and background populations obtained from Besançon simulations of the Galactic stellar populations in the direction of Cepheus. They clearly explain the two-horn shape of the color distribution of 2MASS stars. The horn around $J-H \simeq 0.5-$ 0.8 consists of G-K-M MS background stars (red locus), A-F MS background stars ( green locus), background subgiants (luminosity class IV; purple locus), younger $(<1 \mathrm{Gyr})$ background giants (luminosity class III; black dashed locus around $J-$ $H=0.5$ ), and foreground MS stars (blue locus). The horn around $J-H \simeq 0.8-$ 1.2 , which includes most of the Chandra sources, has older background giants ( $>1 \mathrm{Gyr}$; black dashed locus around $J-H=1.0$ ). The solid black locus covering heavily obscured stars with $A_{V} \gtrsim 5$ mag contains a mixture of embedded PMS stars and highly reddened background MS stars, subgiants, and giants.
} 


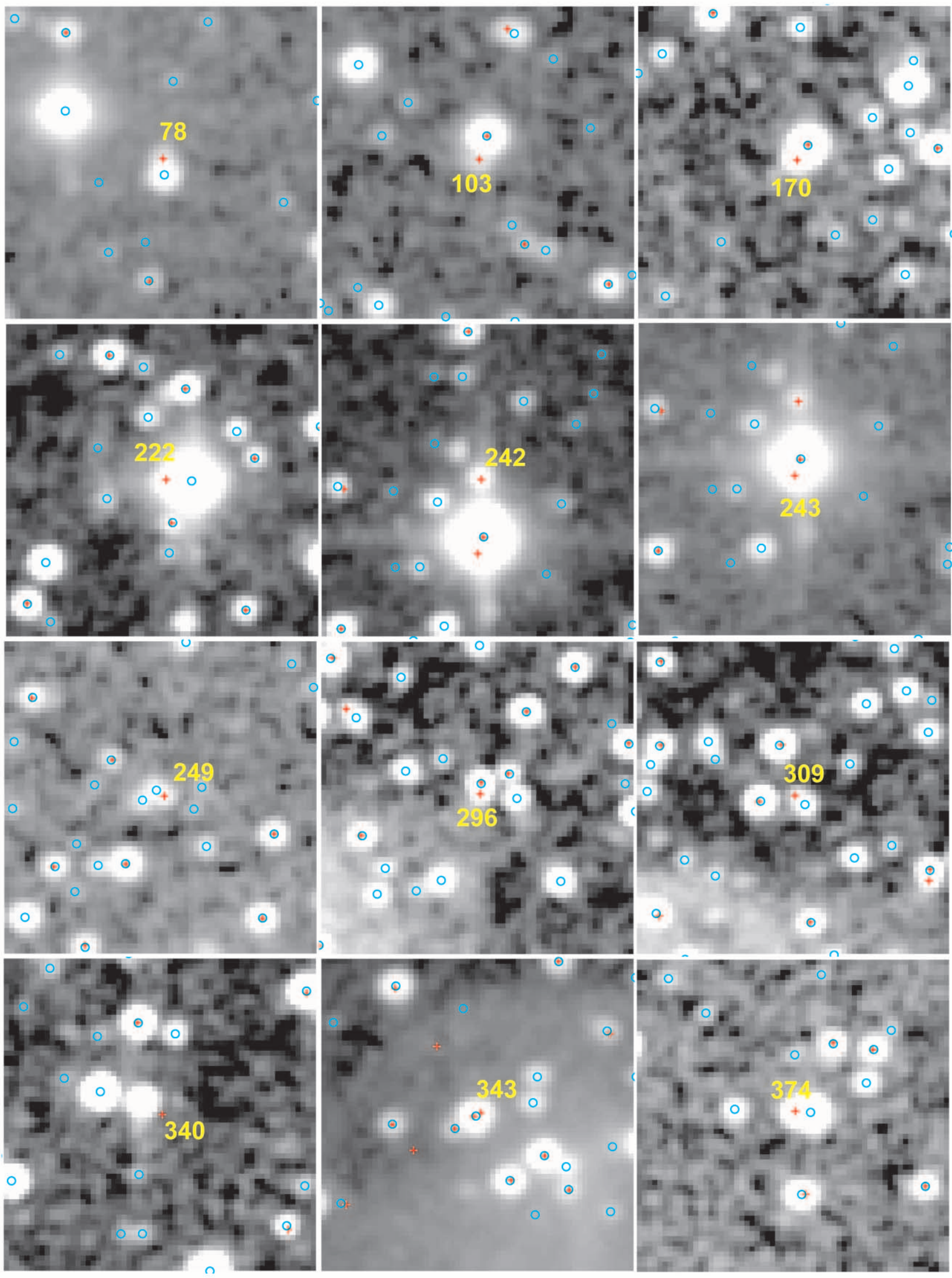

FIG. 3. - Lightly absorbed Chandra stars that are not resolved in the 2MASS catalog. Each panel shows a $1^{\prime} \times 1^{\prime}$ neighborhood $\left(1\right.$ pixel $\left.=1^{\prime \prime}\right)$ around Chandra sources (marked by red plus signs and labeled with their source numbers in yellow) lying in close proximity to bright 2MASS stars. Stars registered in the 2MASS catalog are marked by cyan circles. 


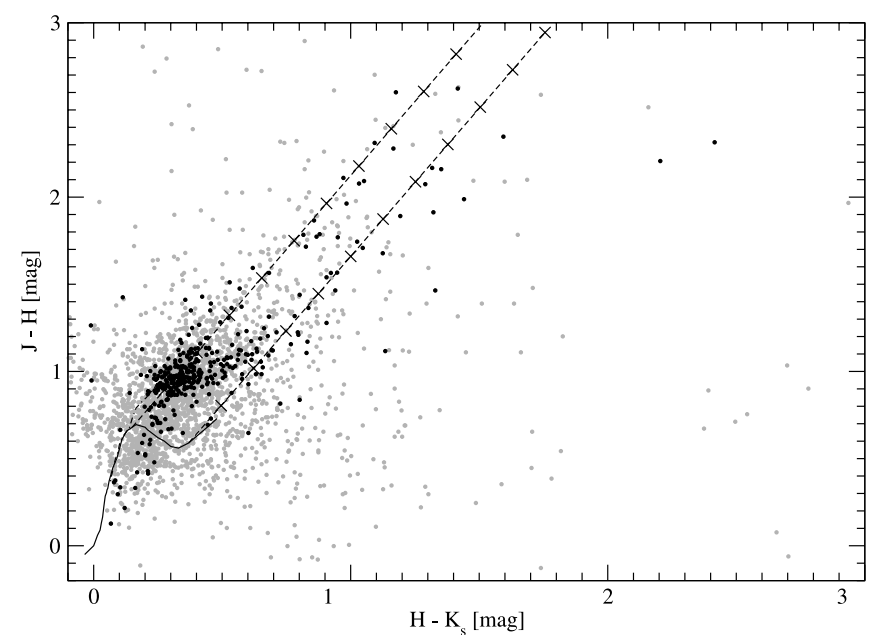

FIG. 4.-NIR color-color diagram shows $J H K$ colors of all ( 2700) 2MASS sources (gray dots) inside the Chandra ACIS-I field, and 385 2MASS sources with possible Chandra counterparts (black dots). The solid and dot-dashed curves show sites of intrinsic $J \mathrm{HK}_{s}$ colors of MS and giant stars, respectively. The dashed lines are reddening vectors originating at M0 V (left line) and M6.5 V (right line), and marked at intervals of $A_{V}=2 \mathrm{mag}$.

very few counts while a few may have thousands of counts. Consequently, no single method is well adapted to characterization of source spectra and fluxes. We describe here three different approaches applied to three flux levels, which are summarized in Figure 6: for 174 sources with $>20$ counts (sixth row), we perform regressions to spectral models giving direct estimations of plasma temperatures, line-of-sight absorption, and broadband fluxes; for 212 weak sources with absorptions estimated from NIR colors $^{7}$ (seventh row), we perform regressions with plasma temperature as a parameter; and for 45 weak X-ray sources without $A_{V, \text { IR }}$ estimates (eighth row), we obtain nonunique spectral fits for the sole purpose of estimating observed broadband fluxes. This last category of sources mostly consists of sources unrelated to the Cepheus star formation region ( $\S \S 5.1$ and 5.2). Resulting spectral fits and broadband luminosities appear in Table 3. Following Getman et al. (2005b), we report observed hard-band (2-8 keV; $\left.\log L_{h}\right)$ and full-band $\left(0.5-8 \mathrm{keV}\right.$; $\left.\log L_{t}\right)$ luminosities, and intrinsic luminosities after correction for absorption $\left(\log L_{h, c}, \log L_{t, c}\right)$. We omit soft-band luminosities due to the absorption seen in virtually all sources.

\subsection{Spectral Fits for Sources with Greater Than 20 Counts}

For 174 sources with greater than 20 net counts (154 lightly absorbed and 20 heavily absorbed), automated spectral fitting was performed using AE and XSPEC (Arnaud 1996). We applied a single-temperature optically thin thermal plasma emission model (Smith et al. 2001; APEC) assuming a uniform density and 0.3 times solar elemental abundances, which is typical of young stellar X-ray sources (e.g., Imanishi et al. 2001; Feigelson et al. 2002). To explore the whole range of various alternative models that successfully fit the data, we performed spectral fits on grouped (with different minimum numbers of grouped counts, starting from 1 ,

\footnotetext{
${ }^{7} N_{\mathrm{H}, \mathrm{IR}}$ was derived from $A_{V, \text { IR }}$ reported in Table 2 using the relationship $N_{\mathrm{H}}=2.0 \times 10^{21} A_{V}$ (Ryter 1996).
}

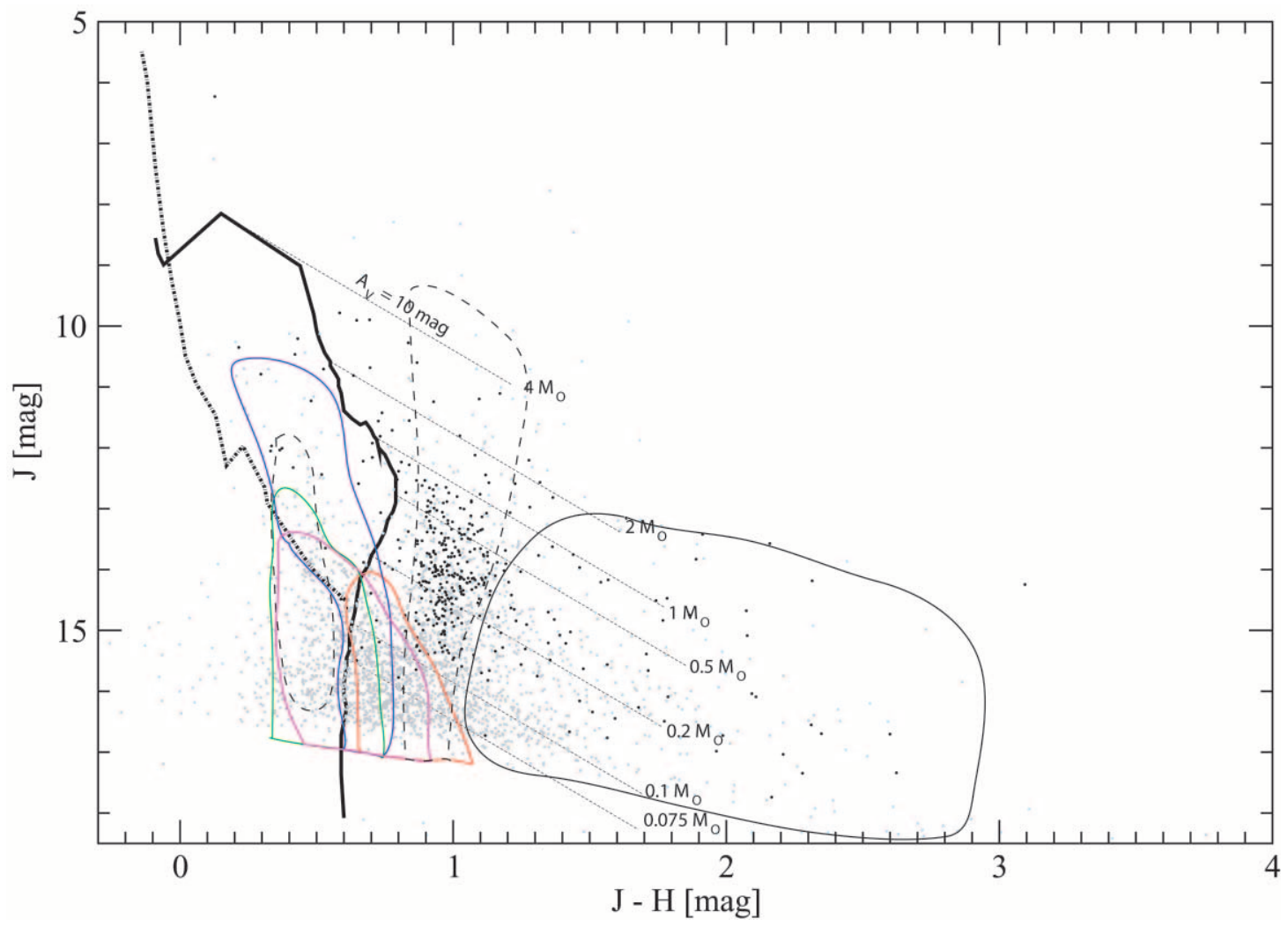

FIG. 5.-NIR color-magnitude diagram for the same set of sources as in Fig. 4. Black dots show Chandra sources with $2 \mathrm{MASS}$ counterparts, and gray dots show all 2MASS sources in the field. Thick black lines give the unabsorbed ZAMS (dotted line) and 1 Myr isochrones (solid line), from which $A_{V} \sim 10$ mag reddening

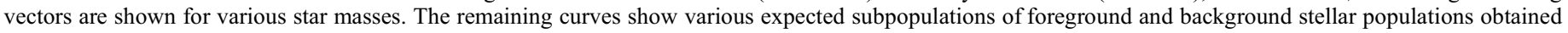
from the Besançon simulations. Details are given in footnote 6. 


\begin{tabular}{|c|c|c|c|c|c|c|c|c|}
\hline Total number of detected X-ray sources: & \multicolumn{8}{|c|}{431} \\
\hline $\begin{array}{l}\text { "Soft" }(\text { MedE }<2 \mathrm{keV})+\text { "Hard" } \\
(\text { MedE }>2 \mathrm{keV}) \text { : }\end{array}$ & \multicolumn{8}{|c|}{$348+83$} \\
\hline "Bright" (>20cnts) + "Weak" (<20cnts): & \multicolumn{4}{|c|}{$154+194$} & \multicolumn{4}{|c|}{$20+63$} \\
\hline $\begin{array}{l}\text { "Id" (identified in 2MASS) + "NoId" (not } \\
\text { identified in 2MASS): }\end{array}$ & \multicolumn{2}{|c|}{$147+7$} & \multicolumn{2}{|c|}{$187+7$} & \multicolumn{2}{|c|}{$16+4$} & \multicolumn{2}{|c|}{$35+28$} \\
\hline $\mathrm{N}_{\mathrm{H}, \mathrm{IR}}$ available + no $\mathrm{N}_{\mathrm{H}, \mathrm{IR}}$ : & $129+18$ & $0+7$ & $177+10$ & $0+7$ & $16+0$ & $\phi+4$ & $35+0$ & $0+28$ \\
\hline $\begin{array}{c}\text { "Bright" sources, spectral results from } \\
\text { XSPEC fits of differently grouped spectra } \\
(174) \text { : }\end{array}$ & \multicolumn{2}{|c|}{154} & & & \multicolumn{2}{|c|}{20} & & \\
\hline $\begin{array}{l}\text { "Weak" sources with } \mathrm{N}_{\mathrm{H}, \mathrm{IR}} \text {, spectral } \\
\text { results from a grid }\left(\mathrm{N}_{\mathrm{H}, \mathrm{IR}} \text { versus MedE) }\right. \\
(212) \text { : }\end{array}$ & & & 177 & & & & 35 & \\
\hline $\begin{array}{l}\text { "Weak" sources with no } \mathrm{N}_{\mathrm{H}, \mathrm{IR}} \text {, spectral } \\
\text { results from XSPEC fits of ungrouped } \\
\text { spectra (45): }\end{array}$ & & & $\begin{array}{c}10 \\
\vdots \\
\end{array}$ & 7 & & & & $\begin{array}{c}28 \\
\vdots \\
\end{array}$ \\
\hline & 13 poșisib & e field & $\begin{array}{l}\text { tars: } \\
+\quad 9\end{array}$ & & 24 poss & ${ }_{3}^{i} \mathrm{AC}$ & $\mathrm{s}$ on $\mathrm{CC}$ & s $\begin{aligned} 1-3 \\
21\end{aligned}$ \\
\hline
\end{tabular}

FIG. 6. - X-ray source classification. The first five rows classify sources based on X-ray photometry and NIR counterpart photometry information. The next three rows indicate classes of sources with different spectral analysis methods applied. The last row shows classes of sources likely unrelated to the Cepheus region derived in $\S \S 5.1$ and 5.2 .

TABLE 3

X-Ray Spectroscopy and Luminosities

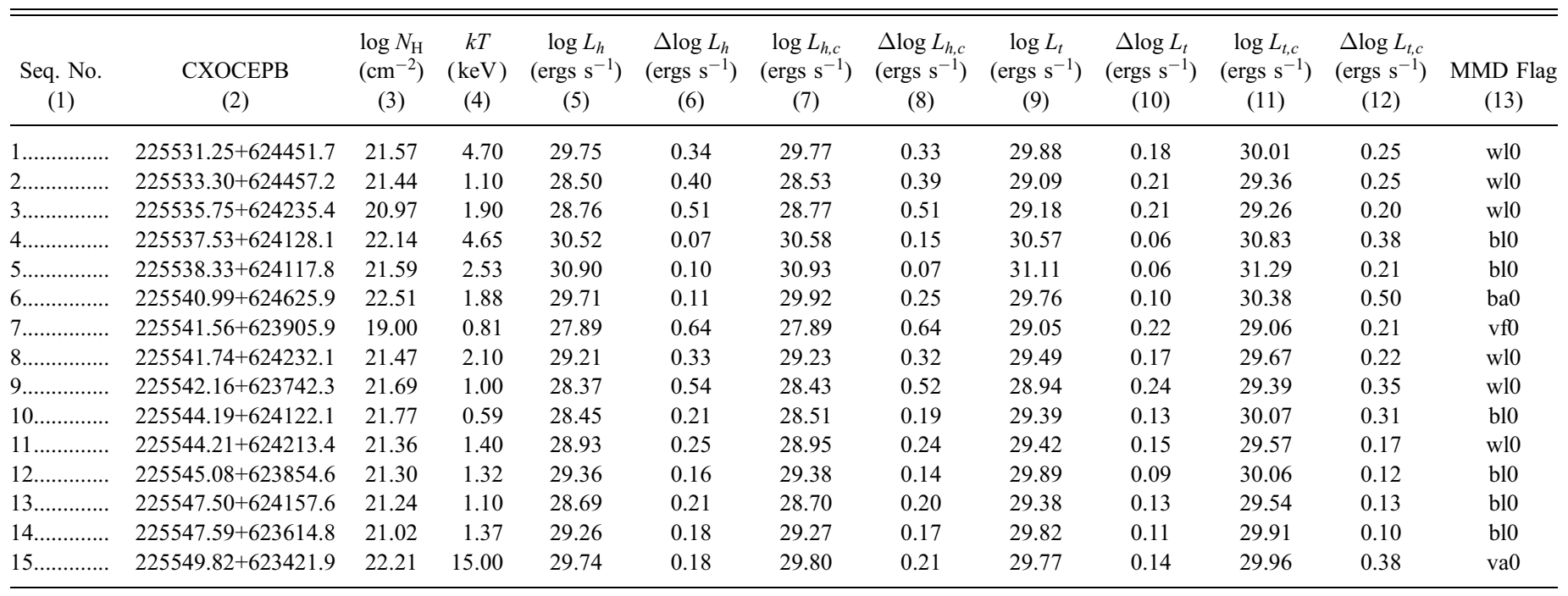

Notes.-Col. (1): X-ray source number. Col. (2): IAU designation. Cols. (3)-(4): Estimated column density and plasma energy. Cols. (5)-(12): Observed and corrected for absorption X-ray luminosities, obtained from our spectral analysis (see $\S 4$ ), and their errors estimated from simulations (see $\S 4.3$ ); $h=$ hard band $(2.0-$ $8.0 \mathrm{keV}), t=$ total band $(0.5-8.0 \mathrm{keV}), c=$ corrected for absorption. Col. (13): Three-part flag summarizing spectral method, source membership, and $K$-disk star information as follows: $\mathrm{b}$ - - = spectral results from spectral fits of 174 relatively "bright" sources with $>20$ net counts; w - - = spectral results from $N_{\mathrm{H}, \mathrm{IR}}-\mathrm{MedE}$ interpolation for 212 "weak" sources with known $N_{\mathrm{H}, \text { IR }} ; \mathrm{v}$ - - = spectral results from fits of ungrouped spectra for 45 "weak" sources with unknown $N_{\mathrm{H}, \text { IR }}$; - e - = possible members of the embedded cluster; - 1 - = possible members of the lightly absorbed cluster; - $\mathrm{o}-=$ other possible members, found in the molecular cloud, not related to the embedded cluster; - a - = AGN candidates; - $\mathrm{f}$ - = field star candidates; - - $\mathrm{d}=$ possible $K$-band excesses associated circumstellar disks; - 0 = stars with no $K$-band excesses or unknown. Table 3 is published in its entirety in the electronic edition of the Supplement. A portion is shown here for guidance regarding its form and content. 
using the $\chi^{2}$-statistic ${ }^{8}$ ) as well as on ungrouped (using the $C$-statistic) spectra. We then performed a visual inspection of all spectral fits to choose the "most appropriate" spectral fit based on subjective judgment, as outlined below.

For lightly absorbed sources (roughly, sources with median energy MedE $<2 \mathrm{keV}$ ), data can often be successfully fit with two or more qualitatively different spectral models. Spectral fits of five sources with 20-23 net counts are shown in Figure 7 to exemplify this common ambiguity in spectral fitting. Sources can often be successfully fit by two different types of models: high $N_{\mathrm{H}}$ and low $k T$, or lower $N_{\mathrm{H}}$ with higher $k T$. Source 167 can be fit by three different classes of models. This problem was also noted by Getman et al. (2005b) for the ONC (the Chandra Orion Ultradeep Project [COUP]), where many more photons are available. In these situations, we adopted a model in which the plasma energy most closely matches the typical energy of PMS stars in Orion: for unabsorbed stars with NIR counterparts, the median plasma temperature (from single-temperature fits) is $k T \sim 1.6 \mathrm{keV}$; for absorbed stars with NIR counterparts, $k T \sim 2.6 \mathrm{keV}$; and for absorbed stars without NIR counterparts, $k T \sim 4.4 \mathrm{keV}$ (Getman et al. 2005a). In Figure 7 we thus preferred the right-hand panel fits. For highly absorbed sources (all except four with $>30$ net counts), data with different groupings are successfully fit by a similar class of models (Fig. 8), and we arbitrarily report a fit from one of the available groupings.

\subsection{Spectral Fits for Sources with Less Than 20 Counts}

Figure 9 compares absorption column densities derived from our X-ray spectra of bright (NetFull $>20$ counts) sources with those derived from the 2MASS $J$ versus $J-H$ color-magnitude diagram. NIR sources were dereddened to the 1 Myr PMS isochrone. We see that the X-ray and NIR absorption columns are statistically equivalent; no bias is seen and scatter is generally within \pm 0.3 in $\log N_{\mathrm{H}} \cdot{ }^{9}$ On this basis, we can reliably substitute $\log N_{\mathrm{H}, \text { IR }}$ for $\log N_{\mathrm{H}, \mathrm{X} \text {-ray }}$ in spectral fitting of 212 fainter sources (177 soft and 35 hard) that have 2MASS NIR photometry.

To estimate plasma temperatures in these faint sources, we use the median energy of the extracted photons. The COUP study showed that MedE is a predictor of $\log N_{\mathrm{H}}$ in strong PMS stars, with a weaker dependence on $k T$ superposed (Feigelson et al. 2005). To test this method, we simulated a grid of absorbed plasma models (wabs*APEC) using the fakeit command in XSPEC. We then passed simulated spectra through AE to perform photometric analysis, including calculation of MedE. For the 212 faint sources with NIR photometry, we fixed column densities to $N_{\mathrm{H}, \mathrm{IR}}$ and placed them on the simulated ( $N_{\mathrm{H}} \mathrm{vs}$. MedE) plane to roughly estimate their plasma energies. With the $N_{\mathrm{H}}$ and $k T$ parameters frozen, we then performed XSPEC fits to derive the spectrum normalization and to calculate broadband X-ray fluxes. Examples of these spectral fits are shown in Figures 10 and 11. The resulting spectral fits appear satisfactory, even for very weak sources of as few as $3-5$ net counts.

Finally, we consider the 45 weak sources ( 17 soft and 28 hard) that lack sufficient 2MASS photometry to estimate $N_{\mathrm{H}, \mathrm{IR}}$. Most of those sources are probably unrelated to the Cepheus region (see $\S 5$ ). Here we report one of the possible spectral fits from a wide range of models in order to integrate under the model and to estimate observed broadband fluxes. The spectral models for these 45 weak sources are used here simply as spline fits with no

\footnotetext{
${ }^{8}$ We tested $\chi^{2}$-statistics with different error-weighting methods, such as standard, Gehrels, and Churazov, and found that the best-fit values of the model parameters remain similar for a spectrum of the same grouping.

${ }_{9}$ A similar correlation exists for COUP data, but it has not been reported.
}
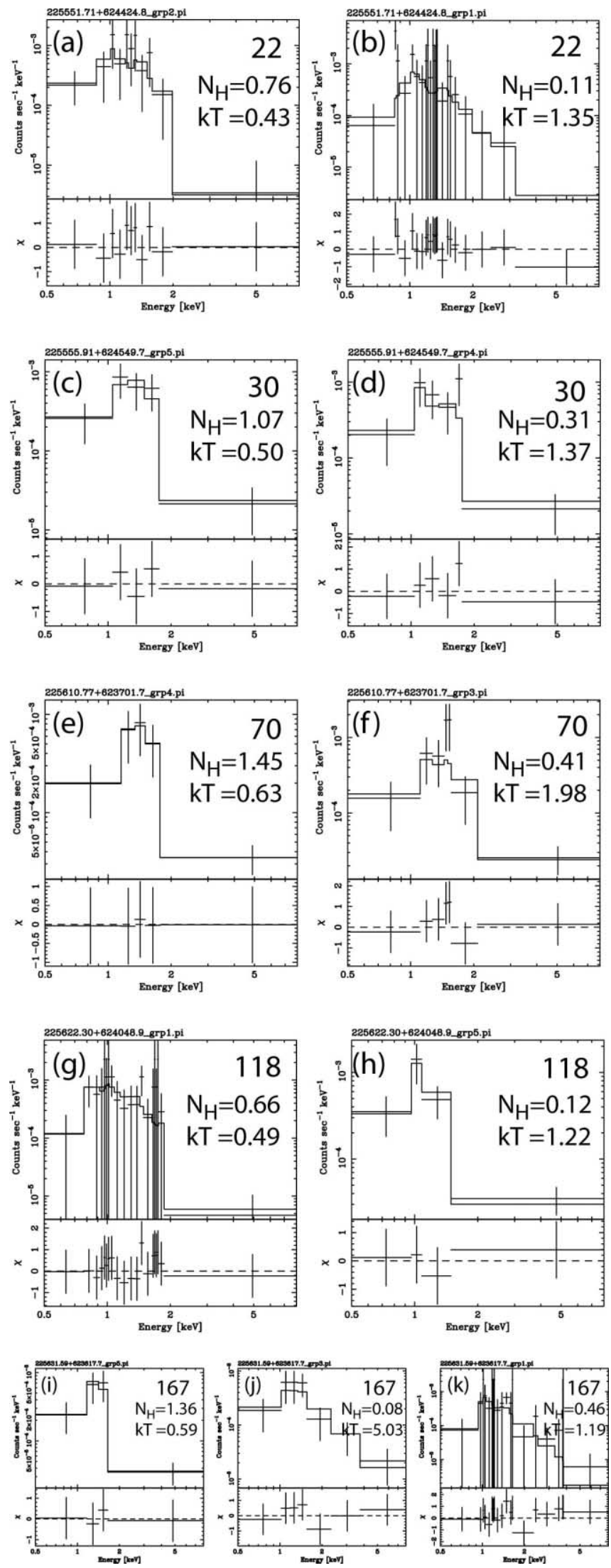

FIG. 7.- Spectra of five lightly absorbed X-ray sources with 20 counts $<$ NetFull $<23$ counts exemplifying a common ambiguity in spectral fitting. Each row shows alternative models that successfully fit the data; the right panel in each row shows the fit we use with $k T$ values typical for COUP PMS stars. 

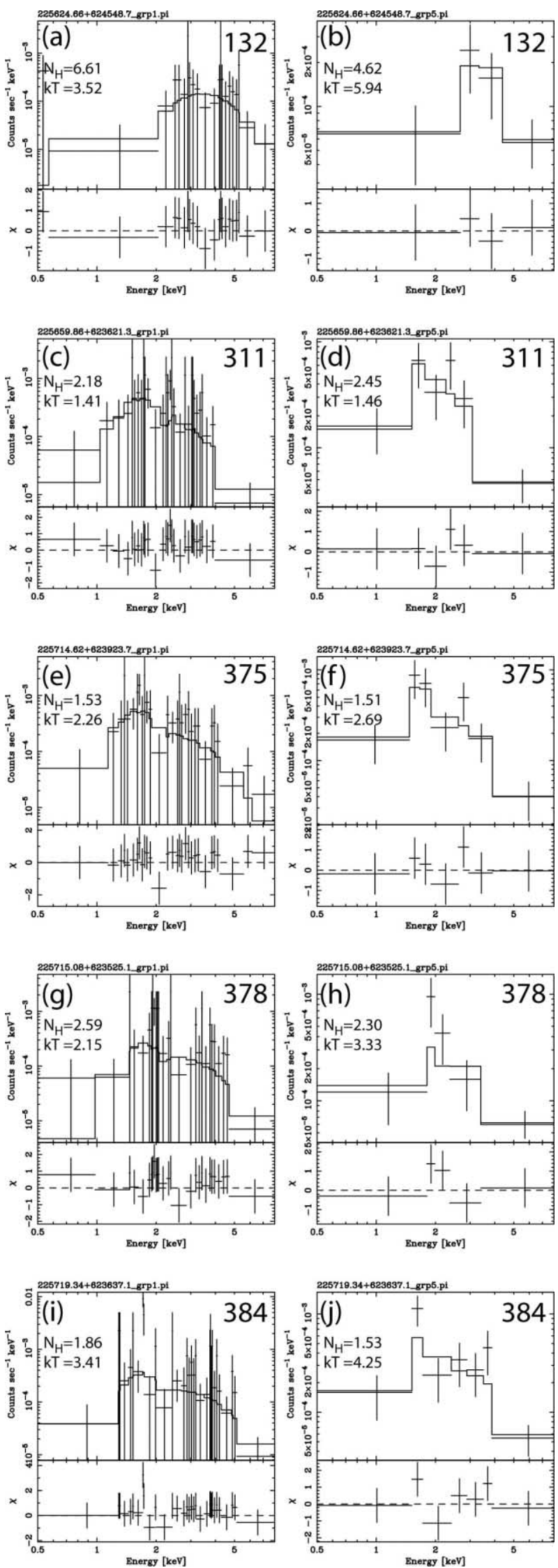

FIG. 8.-Spectra of five heavily absorbed X-ray sources with NetFull $>20$ counts exemplifying stable spectral fitting over different grouping schemes. The finest grouping is to the left, and the coarsest available grouping is to the right. As a final fit for highly absorbed sources, we thus arbitrarily report a fit from one of the available groupings.

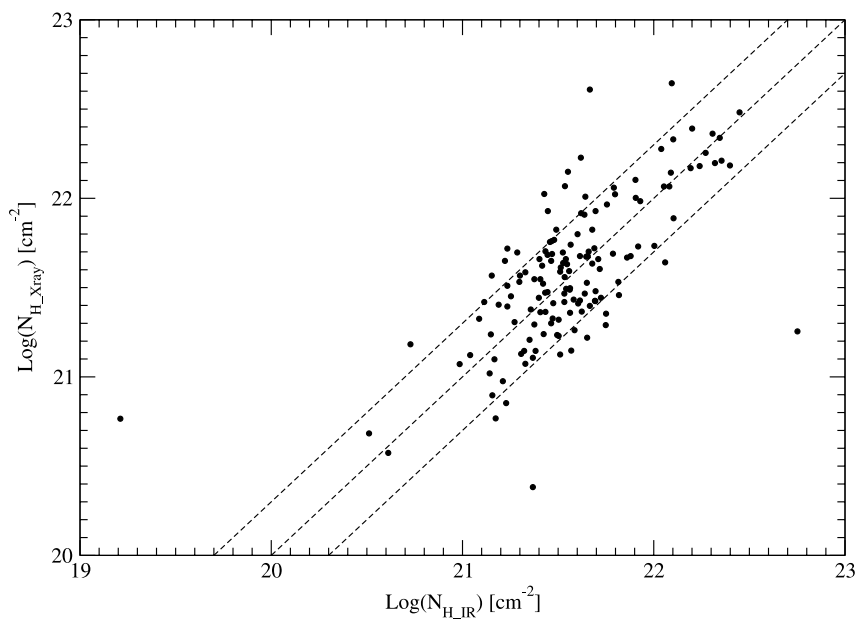

FIG. 9. - Plot of absorbing column densities obtained from X-ray spectral fits $\left(N_{\mathrm{H}, \mathrm{X} \text {-ray }}\right)$ against column densities obtained from NIR photometry $\left(N_{\mathrm{H}, \mathrm{IR}}\right)$. The sample consists of 129 bright soft and 16 bright hard sources (see Fig. 6, row 5). $N_{\mathrm{H}, \text { IR }}$ is derived from the visual absorption $A_{V}$ using $N_{\mathrm{H}}=2.0 \times 10^{21} A_{V}$ (Ryter 1996), where $A_{V}$ is obtained by dereddening source colors to the 1 Myr PMS isochrone in the 2MASS color-magnitude diagram. The dashed lines show $\log \left(N_{\mathrm{H}, \mathrm{X} \text {-ray }} / N_{\mathrm{H}, \mathrm{IR}}\right)$ values of $-0.3,0$, and 0.3 .

astrophysical meaning, mainly to provide future researchers with the ability to derive much more reliable observed X-ray fluxes. Table 3 reports thermal models for all 45 sources; Table 4 reports power-law models for 21 of these sources (see Fig. 6, last row) that are AGN (active galactic nucleus) candidates (see $\S$ 5.1).

\subsection{Flux Uncertainties}

Formal errors on X-ray luminosities for young stellar members of the Cepheus region have been estimated through Monte Carlo simulations. For each source, (1) we randomly draw a distribution of $\log N_{\mathrm{H}}$, composed of 1000 points, with the mean equal to that reported from spectral analysis and $\sigma \sim 0.3$, obtained in $\S 4.2 ;(2)$ we randomly draw a count rate (CR) Poisson distribution of 1000 points with the mean equal to the measured value; (3) using the MARX ray-trace simulator, we simulate a very bright source with the spectral source's characteristics, $N_{\mathrm{H}}$, and $k T$, we slice the simulated source into thousand of chunks, comprising the number of counts of the real source, and we derive the characteristic distribution of the source's median energy (MedE); and (4) we pass the obtained distributions of $\log N_{\mathrm{H}}, \mathrm{CR}$, and MedE through the simulated database of optically thin plasma models (see $\S 4.2$ ) to report the standard deviations of X-ray fluxes in Table 3.

The simulations indicate that $1 \sigma$ errors on MedE are in general better than $0.2 \mathrm{keV}$ for brighter (NetFull $>20$ counts) sources but become as large as $\sim 1 \mathrm{keV}$ for weaker sources. X-ray observed luminosities $L_{t}\left(L_{h}, L_{h, c}\right)$ are known to better than $0.2(0.3$, $0.3)$ dex for brighter sources but become as uncertain as $0.3(0.6$, 0.6) dex for weaker sources (Fig. 12). The uncertainty on intrinsic total-band luminosity $L_{t, c}$, in addition to count rate, is affected by absorption, but in general for most of the sources it is better than 0.4 dex.

The scientific reliability of these luminosities, however, is usually lower than the formal statistical uncertainty for several reasons. First, all PMS stars exhibit strong temporal variability. From Orion studies, we estimate that $\sim 10 \%$ of the stars will be caught in a flare state during the $8 \mathrm{hr}$ of Cep B exposure (Wolk et al. 2005). Second, the $L_{t, c}$ will systematically underestimate the true emission for obscured or embedded populations, due to the absence of 

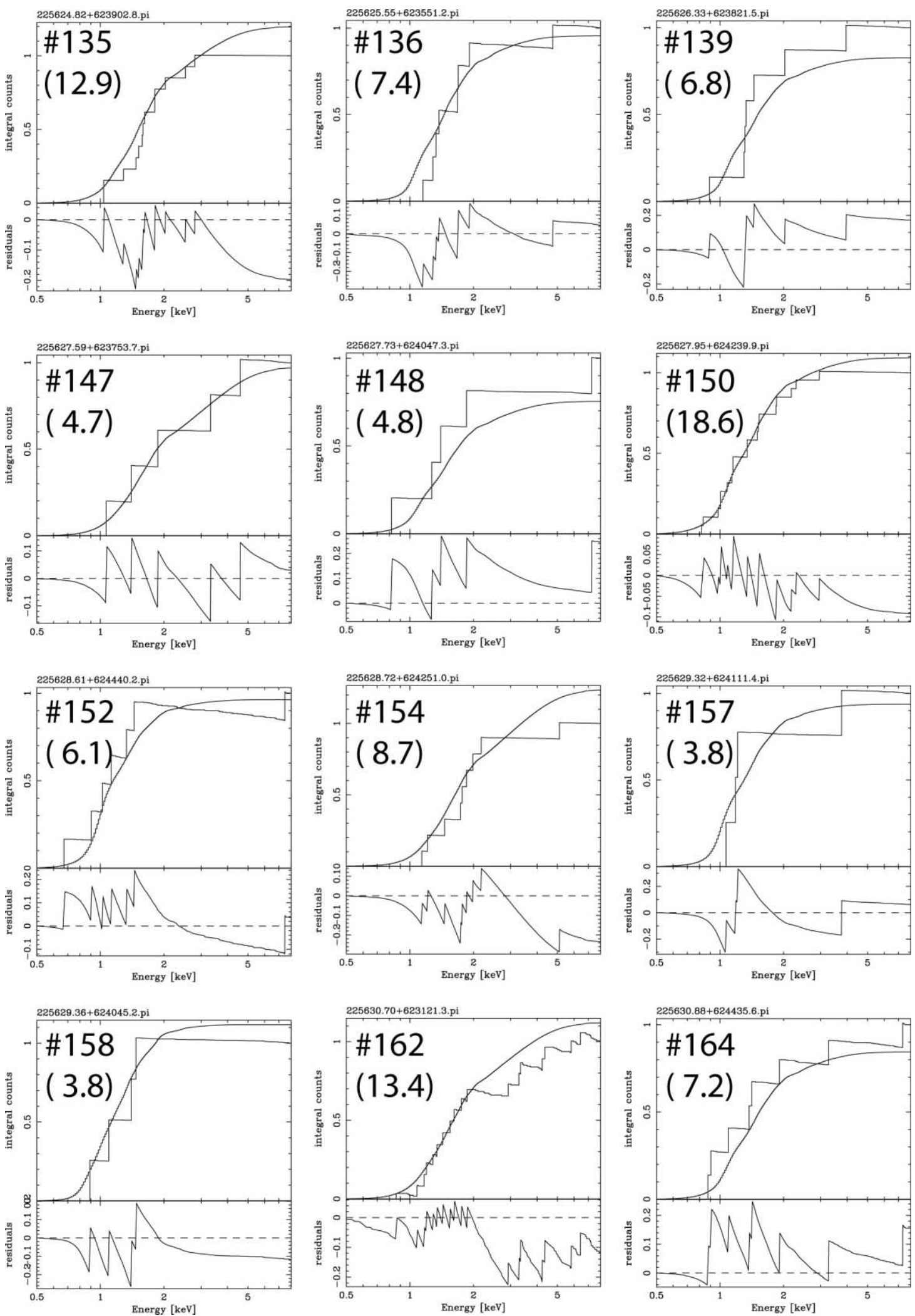

FIG. 10.-Cumulative spectra for a sample of 12 consecutive sources of 177 weak soft sources with available $N_{\mathrm{H} \text { IR }}$ information (Fig. 6, row 7). Upper panels show observed counts (step function) and model spectrum (smooth curve); lower panels show model residuals. $N_{\mathrm{H}, \mathrm{X} \text {-ray }}$ was set equal to $N_{\mathrm{H}, \mathrm{IR}}$. The source number and its net counts appear at the top left. 

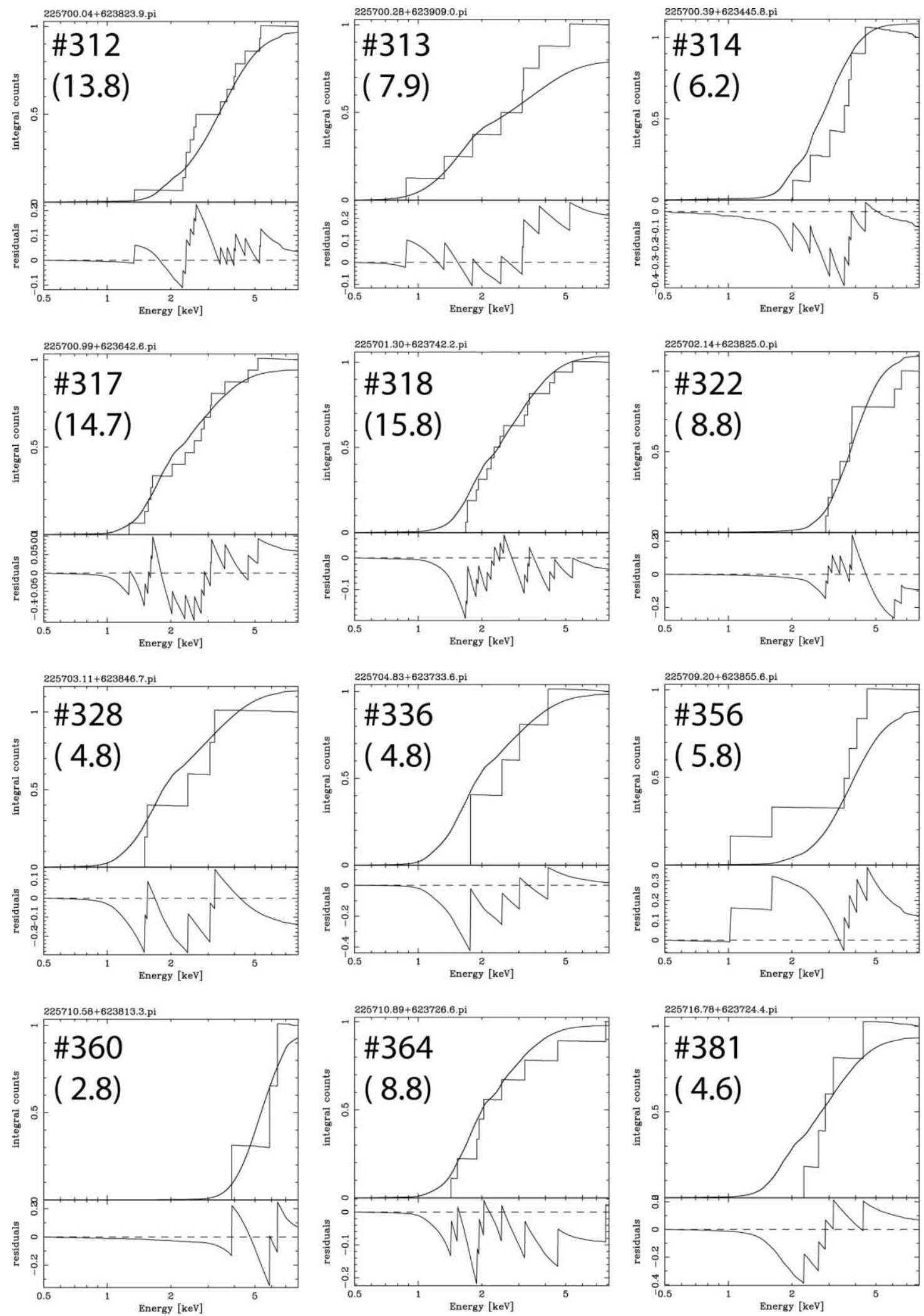

Fig. 11.-Cumulative spectra for a sample of 12 consecutive sources of 35 weak hard sources with available $N_{\mathrm{H}, \text { IR }}$ information (Fig. 6, row 7). Upper panels show observed counts (step function) and model spectrum (smooth curve); lower panels show model residuals. $N_{\mathrm{H}, \mathrm{X} \text {-ray }}$ was set equal to $N_{\mathrm{H}, \text { IR. }}$ The source number and its net counts appear at the top left. 
TABLE 4

Power-Law Fits for Extragalactic Candidates

\begin{tabular}{|c|c|c|c|c|}
\hline $\begin{array}{l}\text { Sequence Number } \\
\text { (1) }\end{array}$ & $\begin{array}{l}\text { CXOCEPB } \\
\text { (2) }\end{array}$ & $\begin{array}{c}\log N_{\mathrm{H}} \\
\left(\mathrm{cm}^{-2}\right) \\
(3)\end{array}$ & $\begin{array}{l}\Gamma \\
(4)\end{array}$ & $\begin{array}{c}\log F_{h} \\
\left(\operatorname{ergs~s}^{-1} \mathrm{~cm}^{-2}\right) \\
(5)\end{array}$ \\
\hline 6. & $225540.99+624625.9$ & 22.05 & 1.71 & -13.73 \\
\hline $15 \ldots$ & $225549.82+623421.9$ & 22.30 & 1.06 & -13.84 \\
\hline$\ldots \ldots$ & $225555.20+624356.6$ & 22.22 & 2.02 & -13.01 \\
\hline .................... & $225557.13+623751.7$ & 22.71 & -1.32 & -14.12 \\
\hline $41 \ldots$ & $225601.45+623923.1$ & 22.11 & 0.11 & -14.13 \\
\hline 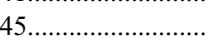 & $225603.10+623714.7$ & 23.11 & 1.17 & -14.13 \\
\hline $64 \ldots \ldots$ & $225608.82+623440.5$ & 20.00 & -0.11 & -14.27 \\
\hline $86 \ldots \ldots$ & $225614.07+623552.7$ & 21.88 & 1.56 & -14.25 \\
\hline 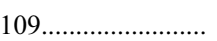 & $225620.22+624321.8$ & 20.00 & 0.68 & -14.36 \\
\hline 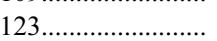 & $225623.11+623557.1$ & 22.53 & 2.93 & -14.08 \\
\hline $132 \ldots$ & $225624.66+624548.7$ & 22.81 & 2.16 & -13.70 \\
\hline $144 \ldots$. & $225627.11+623442.8$ & 23.14 & 9.50 & -14.32 \\
\hline $209 \ldots$ & $225641.33+624259.4$ & 22.17 & 1.48 & -14.10 \\
\hline $215 \ldots$ & $225642.23+624016.3$ & 22.17 & 0.21 & -14.20 \\
\hline $218 \ldots$. & $225642.78+623858.8$ & 20.00 & 0.82 & -14.61 \\
\hline 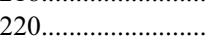 & $225642.80+623211.9$ & 21.48 & 1.41 & -14.30 \\
\hline 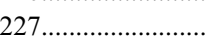 & $225643.88+623832.4$ & 21.60 & 1.46 & -14.55 \\
\hline 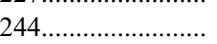 & $225647.41+623057.9$ & 23.22 & 0.95 & -13.41 \\
\hline 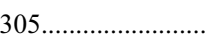 & $225658.56+624434.8$ & 22.66 & 3.01 & -14.39 \\
\hline 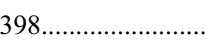 & $225726.61+624628.1$ & 22.46 & 3.11 & -14.37 \\
\hline 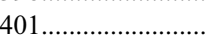 & $225727.44+624516.5$ & 21.67 & 1.07 & -14.29 \\
\hline $415 \ldots \ldots \ldots \ldots \ldots \ldots \ldots \ldots$ & $225734.11+624251.1$ & 22.08 & 0.88 & -13.93 \\
\hline $417 \ldots$ & $225738.06+624238.2$ & 22.87 & 4.20 & -14.21 \\
\hline 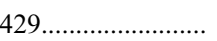 & $225751.63+624813.0$ & 23.38 & 9.46 & -13.86 \\
\hline
\end{tabular}

Notes.—Col. (1): X-ray source number. Col. (2): IAU designation. Cols. (3)(5): Column density, photon index, and observed flux in hard (2.0-8.0 keV) energy band from splinelike fit of ungrouped spectrum. soft-band spectral components. Third, if the distance of $725 \mathrm{pc}$ adopted in this paper were revised to $800 \mathrm{pc}$, quoted in Pozzo et al. (2003), then the PMS stellar X-ray luminosities should systematically be increased by $\simeq 0.1$ dex.

\section{X-RAY SOURCES UNRELATED TO THE CLOUD}

\subsection{Unidentified X-Ray Sources and Extragalactic Contamination}

Out of 83 hard (MedE $>2 \mathrm{keV}$; Fig. 6) sources, 30 have not been identified with 2MASS NIR counterparts (this does not include sources 395 and 403 from $\S 3.2$ ). Twenty-four of these sources without 2MASS counterparts lie outside the Cep B molecular cloud, which covers the southeastern ACIS chip (CCD 0; Fig. 13a). We argue here these are most likely extragalactic sources. No more than three extragalactic sources are likely present behind the dark cloud itself; most of the hard sources in the cloud are concentrated around the PDR ionization front (see Fig. 13a) or in the new heavily absorbed cluster found in the ACIS image ( $(6)$. We therefore omit consideration of extragalactic contamination on the southeastern ACIS chip.

We evaluate the expected contamination by extragalactic X-ray sources using the methods of Getman et al. (2005a). Confining analysis to the hard $(2-8 \mathrm{keV})$ band, we construct Monte Carlo simulations of the extragalactic population by placing artificial sources randomly across the field. Incident fluxes are drawn from the X-ray background $\log N-\log S$ distribution described by Moretti et al. (2003), assuming a power-law photon index distribution consistent with flux dependencies, described by Brandt et al. (2001). The spectrum of each simulated source was passed through a uniform absorbing column density $\log N_{\mathrm{H}} \sim 22.2 \mathrm{~cm}^{-2}$. This is the estimated sum of two components: absorption of $\log N_{\mathrm{H}} \sim 21.7 \mathrm{~cm}^{-2}$ from Earth to the Cepheus region taken from the median of soft X-ray sources away from the dark cloud, and an $\mathrm{H}$ I column density of $\log N_{\mathrm{H}} \sim 22.1 \mathrm{~cm}^{-2}$ through the entire Galactic disk in the direction of Cepheus (Dickey \& Lockman

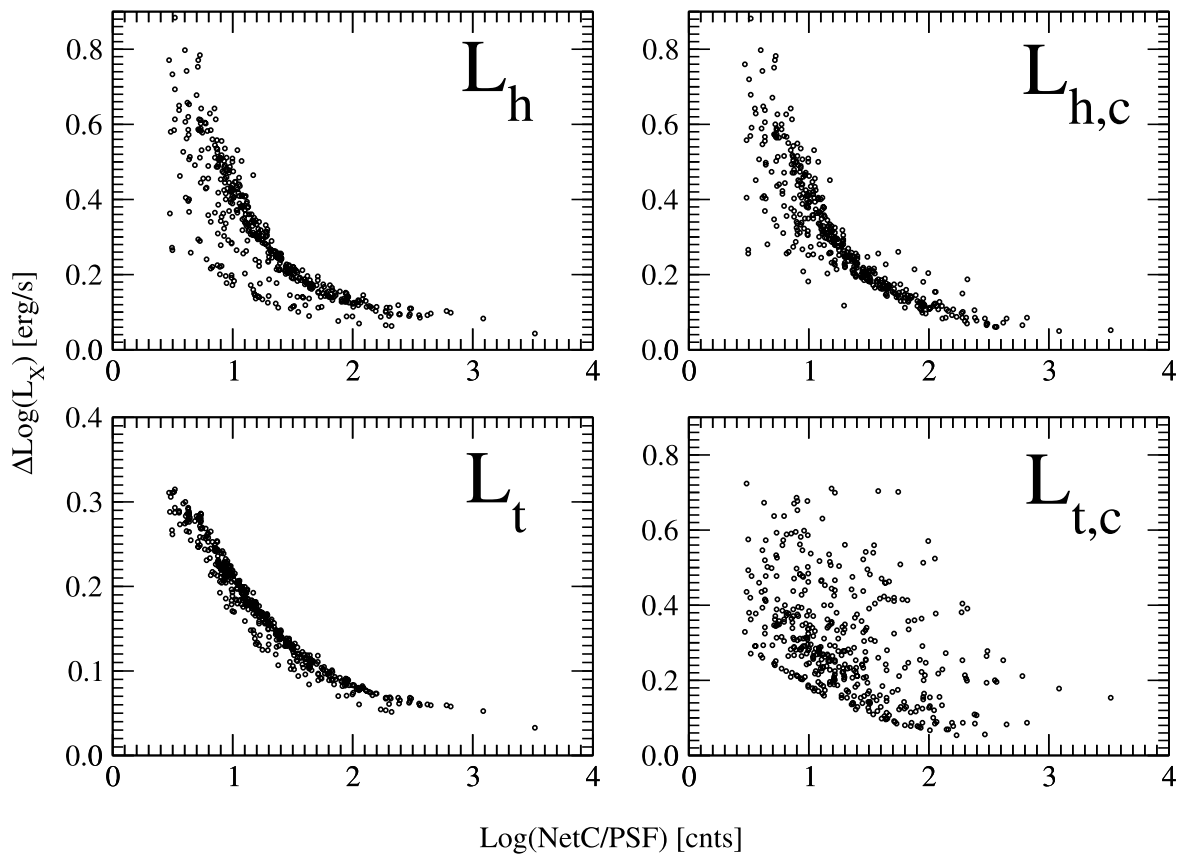

Fig. 12.-Plots of formal errors in X-ray luminosities $\Delta \log \left(L_{\mathrm{X}}\right)$, derived from our Monte Carlo simulations (see $\S 4.3$ ), as a function of source strength $\log$ (NetFull/PSF). The panels show results for four measures of source luminosity: observed hard-band luminosities $\left(L_{h} ; 2.0-8.0 \mathrm{keV}\right)$, hard-band luminosities corrected for absorption $\left(L_{h, c}\right)$, observed total-band luminosities $\left(L_{t} ; 0.5-8.0 \mathrm{keV}\right)$, and total-band luminosities corrected for absorption $\left(L_{t, c}\right)$. 

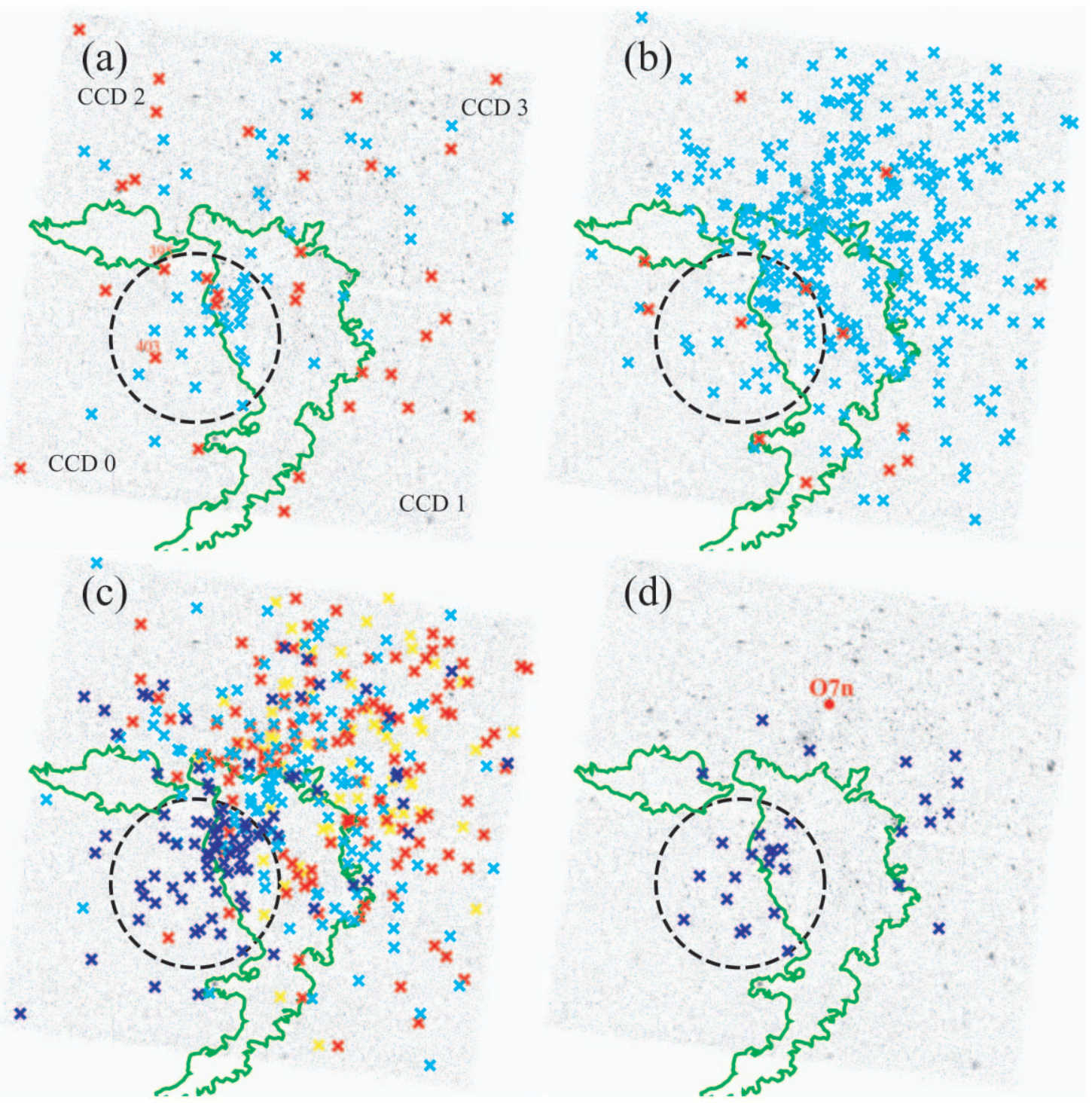

(d)

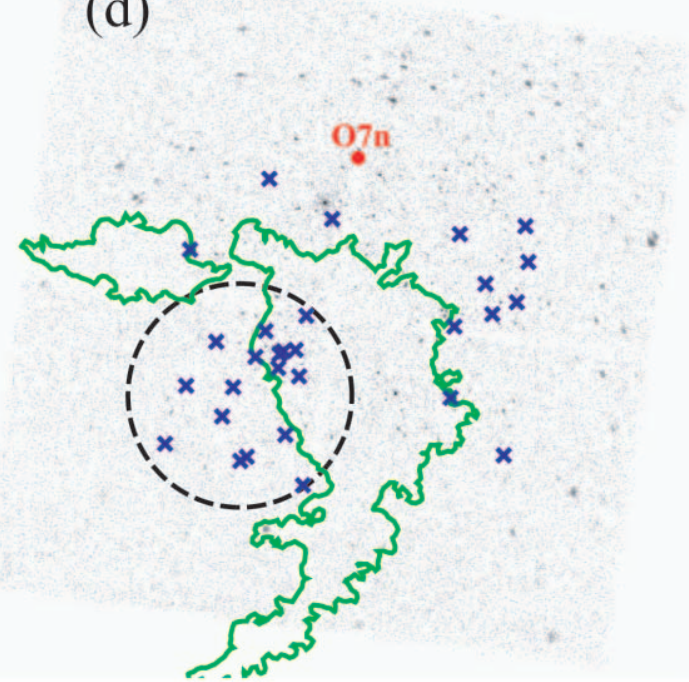

FIG. 13. - Spatial distribution of different classes of X-ray sources superposed on a gray-scale low-resolution image of the $17^{\prime} \times 17^{\prime}$ Chandra field. In each panel, the embedded cluster is outlined by a black dashed circle of $3^{\prime}$ radius. An outline of the $\mathrm{H}$ II region and ionization front seen in the optical DSS image is marked by the green contour. (a) Distribution of all 83 hard (MedE $>2 \mathrm{keV}$ ) X-ray sources, including NIR-identified (cyan crosses) and unidentified (red crosses) sources. The four ACIS-I CCD chips are labeled; the molecular cloud lies mostly on CCD 0. (b) Distribution of all 348 soft (MedE $<2 \mathrm{keV}$ ) X-ray sources, including probable members of the region (cyan crosses) and possible foreground stars (red crosses). (c) $N_{\mathrm{H}}$-stratified distribution of all probable cloud members, i.e., excluding the 24 (red crosses) probable AGNs in CCDs 1 , 2 , and 3 from (a) and the 13 (red crosses) probable field stars from (b). Color code: yellow crosses, $\log N_{\mathrm{H}}<21.3 ;$ red crosses, $21.3<\log N_{\mathrm{H}}<21.6 ;$ cyan crosses, $21.6<$ $\log N_{\mathrm{H}}<21.9$; blue crosses, $\log N_{\mathrm{H}}>21.9$. (d) Distribution of all $29 \mathrm{~K}$-band excess sources (blue crosses). HD 217086 (O7n) is marked by the red dot.

1990). ${ }^{10}$ After applying the local background levels found in our ACIS image, we then remove very weak extragalactic sources that would have fallen below our source detection threshold.

The gray lines in Figure 14 show the predicted $\log N-\log S$ distribution of extragalactic sources from several typical simulations with different detection thresholds. Here $N$ is the total number of extragalactic sources expected to be detected and $S=F_{h}$ is the observed source flux in the hard band. This prediction is compared with the observed hard fluxes of 24 Cepheus hard unidentified sources (Fig. 14, black solid line; values given in Table 4). The simulations predict that a range of 7-17 extragalactic sources would have been detected in these three CCD chips by our data processing, which is roughly half of the 24 observed unidentified hard Cepheus sources. The others may be physically associated

\footnotetext{
10 This was obtained from NASA's HEASARC tool located at http:// heasarc .gsfc.nasa.gov/cgi-bin/Tools/w3nh/w3nh.pl.
}

with the Cepheus region - for example, new embedded sources in the molecular cloud, which may extend toward the southwestern (part of CCD 1) and northeastern (part of CCD 2) parts of the ACIS-I field (Fig. 13a). Since the observed source counts lie somewhat below the simulations for $S<1 \times 10^{-14} \mathrm{ergs} \mathrm{s}^{-1} \mathrm{~cm}^{-2}$, it is plausible that three to five of the hard sources with 2MASS counterparts are bright extragalactic sources rather than PMS stars in the cloud.

As we know that roughly half of the 24 hard unidentified sources are extragalactic contaminants, but we cannot make individual determinations, we flag all 24 sources as possible AGN contaminants (flag "a" in Table 3). They are omitted from further consideration of the Cepheus PMS population.

\subsection{Foreground Stellar Contamination}

Figure 15 shows an expanded view of a portion of the NIR color-magnitude diagram in Figure 5. Thirteen lightly absorbed 


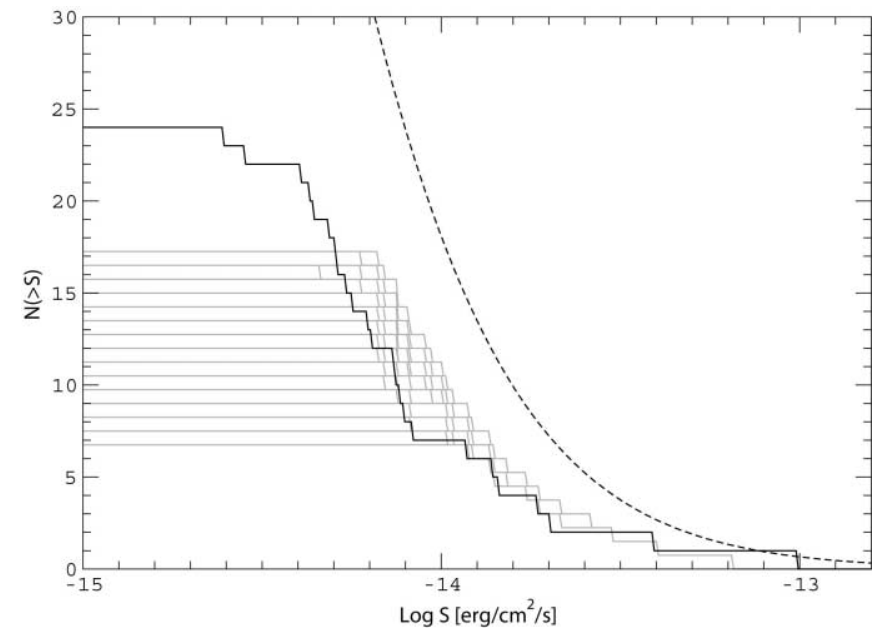

FIG. 14.-Evaluation of extragalactic source contamination using the X-ray source $\log N-\log S$ diagram. $N(>S)$ gives the number of sources in the three CCDs outside of the molecular cloud. $S$ is the source flux in the hard $(2-8 \mathrm{keV})$ band. The black solid line shows the observed distribution for the 24 heavily absorbed sources without 2MASS counterparts. The dashed line shows the expected distribution for an unobscured extragalactic population (Moretti et al. 2003) The gray lines show examples of extragalactic source simulations, which include source spectral variations, large-scale Galactic absorption, absorption local to Cepheus, and variations in the Chandra field X-ray background.

Chandra stars lie between the 1 Myr isochrone and the zero-age main sequence (ZAMS) within the locus of Besançon simulated foreground stars and thus are unlikely to be Cep B cloud members. These are also indicated schematically at the bottom left of the X-ray source classification diagram shown in Figure 6. Although X-ray surveys of star-forming regions are very effective in preferentially detecting PMS stars over MS stars, even a very low detection rate of older stars will lead to interlopers in the $\mathrm{X}$-ray image due to the huge number of field stars. Field stars detected in X-ray surveys will preferentially be younger members of the disk population, as stellar X-ray emission decays rapidly after $\sim 1$ Gyr (Feigelson et al. 2004; Preibisch \& Feigelson 2005).

Half of the field stars in the Besançon simulation have ages greater than $2 \mathrm{Gyr}$, while half have ages ranging from 0.15 to 2 Gyr. Most reside at distances from 400 to 725 pc; $65 \%$ are $\mathrm{M}$ stars, $24 \%$ are $\mathrm{K}$ stars, $9 \%$ are $\mathrm{G}$ stars, and $2 \%$ are more massive. We convolved the Besançon model populations with the $\mathrm{X}$-ray luminosity functions of stars in the solar neighborhood measured from ROSAT surveys (Schmitt et al. 1995; Schmitt 1997; Hünsch et al. 1999). Luminosities were adjusted to account for the different ROSAT and Chandra spectral bands following the stellar hardness-luminosity relation (Güdel et al. 1998). The resulting predicted $\mathrm{X}$-ray flux of the foreground Besançon sample in the $0.5-8.0 \mathrm{keV}$ band is $F_{\mathrm{X}} \sim(2 \pm 1) \times 10^{-13} \mathrm{ergs} \mathrm{s}^{-1} \mathrm{~cm}^{-2}$. Following the same procedure we used for extragalactic sources ( $§ 5.1$ ), we applied our ACIS detection process to these simulated field stars. The results suggest that 5-10 field stars may be detected in the ACIS-I field. A typical Monte Carlo run predicts two F stars, three G-K stars, and four M stars. Half are typically between 450 and $725 \mathrm{pc}$. This prediction may rise to $10-15$ stars if one includes an age dependence to the X-ray luminosities (Preibisch \& Feigelson 2005) into the simulations, since about one-fourth of the stars have ages $<1$ Gyr.

The 13 Chandra sources highlighted in Figure 15 have NIR magnitudes and colors lying within the locus of expected foreground stars. The spatial distribution of the 13 soft-spectrum stars is relatively uniform in the ACIS field (see Fig. 13b, red crosses),

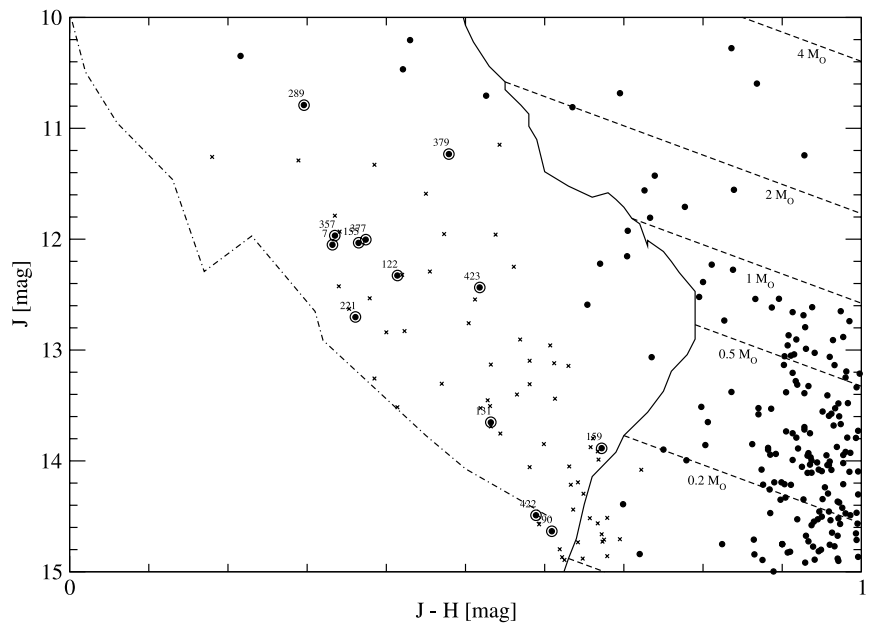

FIG. 15.-Expanded view of the foreground star locus area on the NIR colormagnitude diagram shown in Fig. 5. Chandra sources are marked by black dots. The small crosses show simulated star locations of foreground stars based on the Besançon Galactic star model. Thirteen likely X-ray foreground stars are labeled and outlined by circles.

and they have soft X-ray spectra expected from MS coronal emission (sources with flag "f" for "foreground" in Table 3). We omit these X-ray sources from further consideration of the Cepheus population.

\subsection{Galactic Background Contamination}

The Besançon model is again convolved with X-ray luminosity functions to simulate the number of stars behind the cloud that may enter our X-ray sample. We consider only plausible classes of X-ray emitters (e.g., omitting MS A stars and subgiants) and adopt a limiting magnitude of $V<22$. The model then predicts $\sim 1700 \mathrm{~F}$ dwarfs, $\sim 550 \mathrm{G}$ dwarfs, $\sim 1950 \mathrm{~K}$ dwarfs, $\sim 25 \mathrm{M}$ dwarfs, and $\sim 632$ giants. We again use the dwarf XLFs established for the solar neighborhood (Schmitt et al. 1995; Schmitt 1997). For giants we assume the lognormal XLF of $\sim\langle 28.9\rangle \pm 0.9$, obtained from Table 2 of Pizzolato et al. (2000) for a sample of 120 post-MS latetype stars within 100 pc. Rarely, simulations result in up to five dwarfs and seven giants detected in our Chandra observation, while typical runs suggest no dwarfs and three giants. As we cannot make individual determinations for three possible background giants among our PMS stars, we ignore Galactic background contamination in our further consideration of the Cepheus PMS population. The simulations yield a total typical observed X-ray flux of the Galactic background population of up to $F_{\mathrm{X}} \sim 2 \times$ $10^{-14} \mathrm{ergs} \mathrm{cm}^{-2} \mathrm{~s}^{-1}$ that may be contributing to the X-ray diffuse emission seen in the ACIS-I field.

\section{DISCOVERY OF TWO RICH STELLAR CLUSTERS}

Cepheus B, the hottest CO component of the Cepheus molecular cloud, is located at the northwestern edge of the cloud near the Cepheus OB3 association. The association is thought to be composed of two subgroups of different ages, with the youngest lying closer to the molecular cloud (Sargent 1979). The interface between the molecular cloud and the OB association is delineated by the optically bright $\mathrm{H}$ II region $\mathrm{S} 155$, with ionization fronts bounding the dust/molecular cloud (Felli et al. 1978). Testi et al. (1995) detected four radio structures - one extended source tracing an ionization front and three compact sources inside the molecular cloud - accompanied by three very red NIR objects. They suggested these are manifestations of a previously unrecognized 
young embedded stellar cluster at the interface of the $\mathrm{H}$ II region and the molecular cloud.

Moreno-Corral et al. (1993) claimed the discovery of more than 50 NIR sources, with $60 \%$ of them located close to or within the Cep B molecular cloud. Unfortunately, it seems that positions for those sources reported in their Table 5 are incorrect, as none of them matches known 2MASS or Chandra objects considering any possible systematic shift. Thus we omit the results of this work from further consideration.

Naylor \& Fabian (1999) found more than 50 ROSAT X-ray sources in Cep OB3b; these revealed a low-mass stellar population accompanying the high-mass stars of the OB association. Optical CCD photometry and spectroscopy identified 10 ROSAT sources in their catalog, of which four were confirmed as T Tauri stars and one was suggested as a possible PMS star (Pozzo et al. 2003). Six (five) more optical stars were associated with T Tauri (possible PMS) stars, totaling 10 (6) confirmed T Tauri (possible PMS) stars. The results of Testi et al. (1995), Naylor \& Fabian (1999), and Pozzo et al. (2003) are summarized in Figure 16, which shows the previously known PMS stars lying in the ACIS-I field: four radio sources (squares), three red NIR sources (crosses), 27 of 50 ROSAT sources (diamonds), ${ }^{11}$ and 5 of 16 optical PMS stars (plus signs). The four stars from Pozzo et al. (2003) with Chandra counterparts are 7a (Chandra source 193), 8 (source 156), 12 (source 197), and 106 (source 160). Pozzo et al. (2003) recognized the incompleteness and low spatial resolution of the ROSAT data and anticipated our Chandra X-ray observation, which is capable of reaching a much larger PMS population.

The popular scenario for star birth in the S155/Cep B region (Minchin et al. 1992) is that the ultraviolet (UV) radiation from the O7n star HD 217086, the brightest member of Cep OB3b cluster, ionizes ambient material and induces a burst of star formation. We investigate this model by searching for the next (third) generation of young stars directly behind the ionization front. Figure $13 c$ shows the $N_{\mathrm{H}}$-stratified distribution of the Chandra source population, excluding field and extragalactic candidates, overlaid on an outline of the optical $\mathrm{H}$ il region.

A clear congregation of $\sim 64$ highly absorbed young stellar objects is seen in a $\sim 3^{\prime}(\sim 0.6 \mathrm{pc})$ radius area, delineated by the black circle, at the $\mathrm{H}$ II-molecular cloud interface. The remaining 321 Chandra stars with moderate or low absorption are located outside the molecular cloud, although about nine highly absorbed stars are dispersed throughout the field. Figure $13 d$ shows the distribution of Chandra stars with a $K$-band excess inferred from 2MASS photometry (Fig. 4). These stars are likely to be younger than the stars without bright inner dusty disks. These are preferentially associated with the cluster of highly absorbed Chandra stars: $26 \%$ of stars within the black circle have $K$-band excesses, compared to $4 \%$ elsewhere in the field. We recognize that the UV radiation from the O7n star could evaporate nearby circumstellar disks, but this likely affects only the outer cooler disk regions.

Our findings support the triggered star formation scenario of Minchin et al. (1992) with an unambiguous discovery of the thirdgeneration embedded star cluster forming close to the ionization front. This is based on the spatial concentration of highly absorbed Chandra sources and their high proportion of $K$-band excesses just behind the S155 ionization front. It seems unlikely that this cluster was formed by spontaneous self-gravitational collapse of

\footnotetext{
11 All except one of these ROSAT sources have one or multiple Chandra counterparts. ROSAT source 41 is missed, probably because it lies on a gap between CCD chips in the ACIS array. One of the candidate optical PMS stars similarly lies on a chip gap.
}

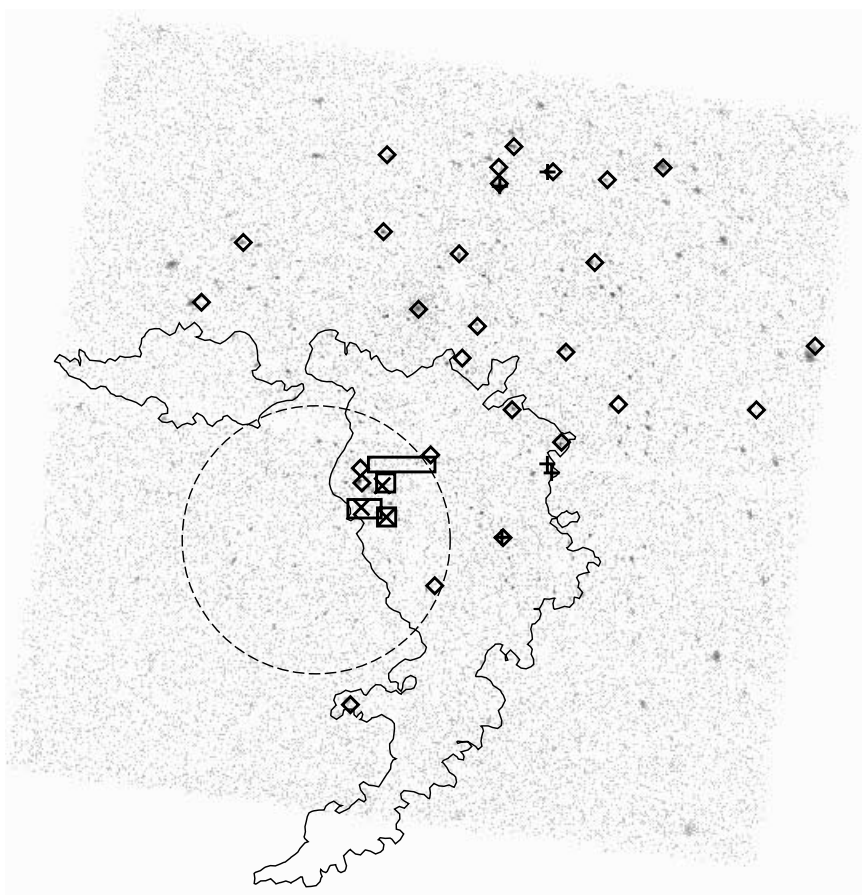

FIG. 16.-Positions of previously reported young members of the region, which fall within the ACIS-I field: four VLA compact radio sources (squares) and three red NIR objects (crosses) from Testi et al. (1995), 27 ROSAT sources from Naylor \& Fabian (1999; diamonds), and five PMS stars with optical spectra from Pozzo et al. (2003; plus signs). Lines are the same as in Fig. 13: the dashed circle outlines the embedded cluster of X-ray stars, and the solid contour outlines the $\mathrm{H}$ II region and ionization front seen in the optical DSS image.

molecular cloud material. We detect $\sim 64$ members of this embedded cluster (henceforth denoted as the "Cep B embedded cluster"), which are marked "e" (= "embedded") in column (13) of Table 3 . The 321 members of the older second generation cluster (hence denoted as "Cep OB3b cluster within ACIS-I field" = "Cep OB3b unobscured cluster") are marked "l" (= "lightly absorbed").

\section{XLFs, KLFs, AND IMFs}

A major goal of studies of young stellar clusters is to quantify and understand the distribution of masses arising from star formation processes (Corbelli et al. 2005). This effort is subject to a variety of difficulties, but as X-ray surveys present different problems than those arising at other wave bands, they can be valuable for IMF studies (Feigelson \& Getman 2005). In regions like Cep B, NIR surveys are overwhelmed by unrelated field stars (e.g., Fig. 4). A common procedure is to subtract statistically NIR source counts from a nearby control field and convert the resulting $K$-band luminosity function (KLF) into masses using theoretical stellar models to estimate a cluster IMF (Lada \& Lada 1995). We use a similar procedure on the Cep B region below ( $\S 7.3$ ). X-ray surveys, on the other hand, suffer relatively little contamination; we find that no more than $5 \%$ of Chandra sources in the Cep B field are likely extragalactic contaminants and no more than $4 \%$ are likely stellar contaminants $(\S 5)$. Infrared surveys also are biased toward selection of young stars with heavy protoplanetary disks, while $\mathrm{X}$-ray surveys are mostly unbiased with respect to disks (although a small bias against accretion is present; Preibisch et al. 2005). X-ray surveys penetrate deeper into obscuring material than optical or NIR surveys, but are less complete in detecting the lowest mass objects. 


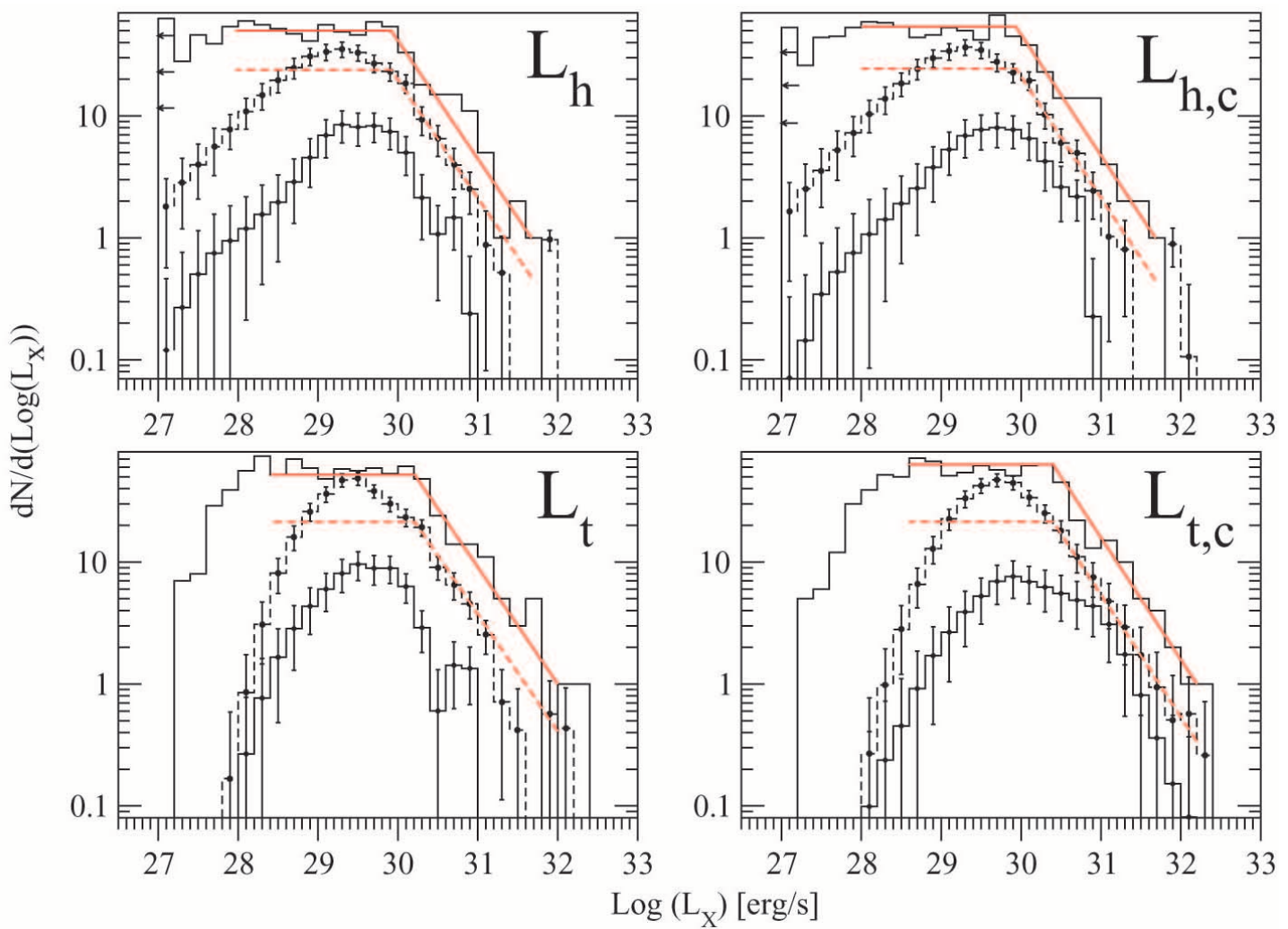

FIG. 17.-Comparison between XLFs of the ONC and Cepheus populations ( $§ 7.1)$. The upper solid histograms show the COUP unobscured cool sample of 839 stars (Feigelson et al. 2005); the middle dashed histogram shows the Cep OB3b unobscured sample of 321 stars; the lower solid histogram shows the Cep B obscured sample of 64 stars. X-ray luminosities are the same as in Fig. 12. Error bars indicate 68\% confidence intervals $(1 \sigma)$ from Monte Carlo simulated distributions when X-ray luminosities are randomly drawn from Gaussian distributions with the sources' measured $\log L_{\mathrm{X}}$ values and estimated errors $\Delta \log L_{\mathrm{X}}$. The red lines are added to aid the eye; they are based on the shape of the ONC XLFs and scaled downward to match the Cep OB3b unobscured population.

In this section, we first examine the empirical stellar XLF of the Cepheus population and compare it to the well-studied ONC XLF (§ 7.1). We then use independently derived masses for Cepheus members to estimate the IMF (§ 7.2) and compare this with an IMF created from the KLF $(\S 7.3)$. The result is surprising: the Cep OB3 cluster - at least the part of it seen in the ACIS-I field - appears to have a deficiency in higher mass stars compared to the ONC and standard IMFs.

\subsection{Cep $O B 3 b$ and $O N C X L F s$}

Comparison of XLFs between poorly characterized young stellar clusters such as Cep OB3b and a well-characterized cluster such as the $\mathrm{ONC}$ can give insight into any missing population of stars. This was done by Grosso et al. (2005) to estimate the total population of the embedded Becklin-Neugebauer-KleinmanLow OMC-1 population. Figure 17 shows such a comparison among the unobscured COUP population (upper solid line), the Cep OB3b population (dashed line with error bars), and the Cep B embedded population (lower solid line with error bars). The ONC sample (= 839 COUP lightly obscured cool stars) consists of COUP cloud members excluding OB stars and sources with absorbing column density $\log N_{\mathrm{H}}>22.0 \mathrm{~cm}^{-2}\left(A_{V} \gtrsim 5 \mathrm{mag}\right)$ (Feigelson et al. 2005). XLF error bars for the Cep OB3b and Cep B embedded populations show the range containing 68\% ( $1 \sigma$ equivalent) from Monte Carlo simulated XLF distributions, where individual X-ray luminosities are randomly drawn from Gaussian distributions with mean equal to the measured source's $\log L_{\mathrm{X}}$ and variances based on observed $\Delta \log L_{\mathrm{X}}$ values. The error bars thus include both the $\sqrt{N}$ counting error and the $L_{\mathrm{X}}$-dependent mea- surement error. Regression lines to COUP's XLFs were added to figures by eye (red solid lines) and scaled vertically to obtain a predicted XLF for the Cep OB3b population (red dashed lines).

It is clear from Figure 17 that the Cep OB3b cluster XLF shows a significant excess of stars around $\log L_{t, c} \sim 29.7, \log L_{t} \sim 29.5$, and $\log L_{h, c} \sim \log L_{h} \sim 29.3$, compared to the scaled ONC XLF. This excess is not related to incompleteness of the current observation. Figure 18 shows $^{12}$ that the completeness limit for the observed total-band luminosity is around $\log L_{t}=29.5 \mathrm{ergs} \mathrm{s}^{-1}$, and is still above $50 \%$ at $\log L_{t}=29.0 \mathrm{ergs} \mathrm{s}^{-1}$. This is far below the range $\log L_{t} \gtrsim 30.2 \mathrm{ergs} \mathrm{s}^{-1}$ over which the ONC and Cep OB 3b XLFs were scaled. We are thus confident that the relative amplitudes of the XLFs in Figure 17 are correct. In fact, the excess of Cep OB3b X-ray stars over the ONC XLF becomes even stronger if the completeness curve from Figure 18 is applied to the incomplete Cep OB3b $L_{t}$ XLF of Figure 17.

Table 5 summarizes total X-ray luminosities for all X-raydetected stars in the Cep OB3b unobscured and Cep B embedded populations within the ACIS-I field. It thus appears that the Cep OB3b population within the ACIS-I field has an XLF with a different shape than seen in the ONC. Instead of a double-power-law

\footnotetext{
12 Fig. 18 is derived from Monte Carlo simulations performed by randomly drawing sources of a given luminosity scattered throughout the ACIS image and applying local background levels found in the image, similar to the AGN simulations in $\S 5.1$. Observed total-band luminosity $L_{t}$ was chosen as it is more readily converted to the observed source count rate assuming a typical distribution of source median energy, and the source significance then is easily calculated. Source significance thresholds are then evaluated to mimic the behavior of our source detection procedure.
} 


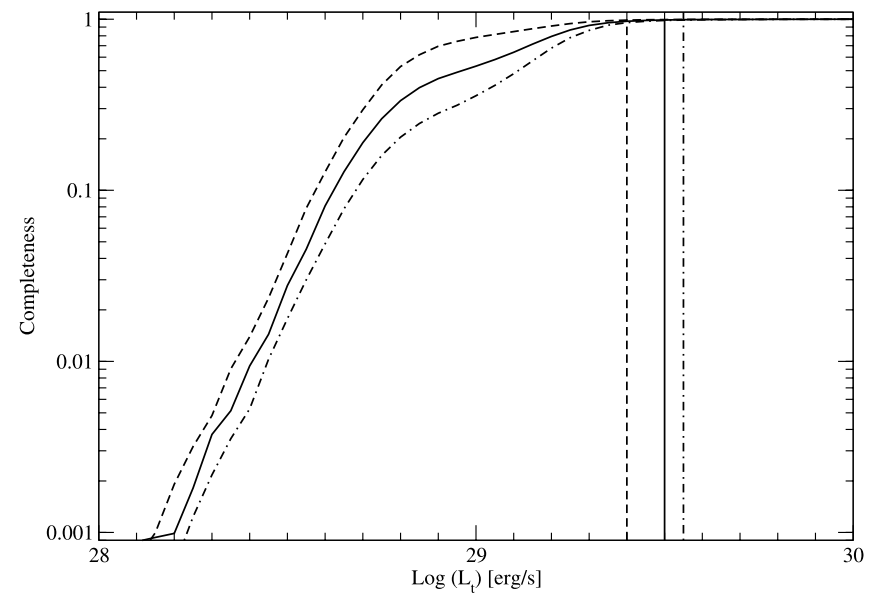

FIG. 18.-Detection probability for a point source at a given observed totalband luminosity, as derived from our Monte Carlo simulations of the Cep OB3 ACIS observation. Three completeness curves correspond to three different detection thresholds: most representative of the current observation (solid curve), more conservative (dot-dashed curve), and less conservative (dashed curve). The three vertical lines indicate corresponding completeness limits: 29.5 (solid line), 29.55 (dot-dashed line), and 29.4 (dashed line).

model with a break around $L_{t, c} \sim 2.5 \times 10^{30} \mathrm{ergs} \mathrm{s}^{-1}$, the Cep OB3b XLF with the sensitivity limit of $\log L_{t, c, \lim } \sim 10^{29} \mathrm{ergs} \mathrm{s}^{-1}$ can be roughly modeled as a lognormal with mean around $\left\langle\log L_{t, c}\right\rangle \sim 29.8 \mathrm{ergs} \mathrm{s}^{-1}$ and standard deviation of $\sigma\left(\log L_{t, c}\right) \sim$ 0.6 . The mean and standard deviation of cluster XLFs are perhaps observation-biased; for example, for more sensitive observations of NGC 1333 and IC 348 with $\log L_{t, c, \lim } \sim 10^{28} \mathrm{ergs} \mathrm{s}^{-1}$, the XLF shapes are wider with a standard deviation of $\sigma\left(\log L_{t, c}\right) \sim$ 0.9 , peaking at lower luminosities of $\left\langle\log L_{t, c}\right\rangle \sim 29.5 \mathrm{ergs} \mathrm{s}^{-1}$ (Feigelson \& Getman 2005).

\subsection{Cep $O B 3 b$ and $O N C I M F S$}

To compare the Cepheus IMF with that of the ONC, we need to adopt compatible procedures for estimating masses. To be consistent with the current work and to derive masses for the whole sample, we rederive ${ }^{13}$ COUP stellar masses here using NIR photometry (Table 10 of Getman et al. 2005b). Similar to Cepheus masses, ONC mass estimates are based on star locations in the $J$ versus $J-H$ color-magnitude diagram and theoretical stellar model tracks derived by Baraffe et al. (1998; for $0.02 M_{\odot} \leq M \leq$ $\left.1.4 M_{\odot}\right)$ and Siess et al. $\left(2000\right.$; for $\left.1.4 M_{\odot} \leq M \leq 7.0 M_{\odot}\right)$. We are aware that these mass estimates are subject to significant uncertainties and may be incompatible with masses obtained by other methods such as optical spectroscopy. However, here we are

\footnotetext{
13 Table 9 of Getman et al. (2005b) provides masses for half of the COUP sample based on optical spectroscopy.
}

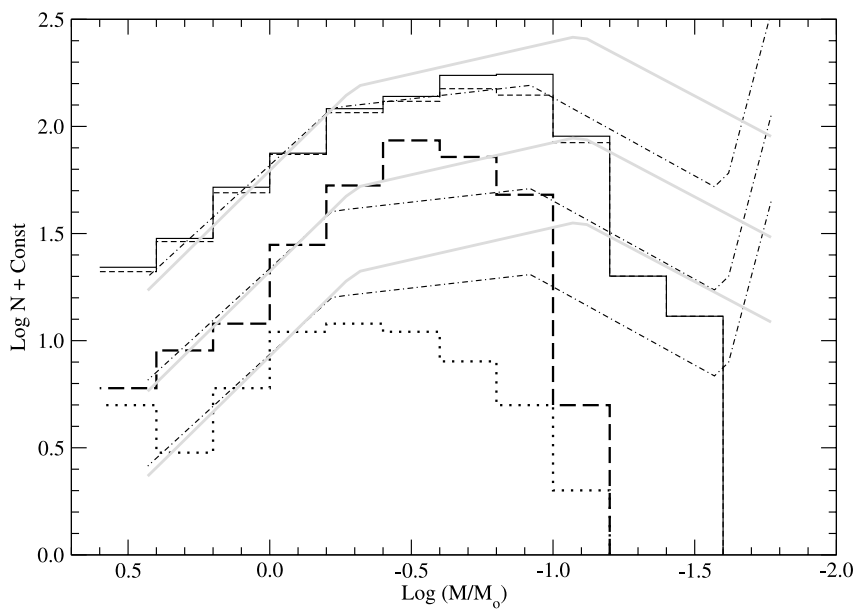

FIG. 19.-Comparison between IMFs of the ONC and the Cepheus X-ray sources, where stellar masses for both clusters are derived from NIR photometry ( $§ 7.2$ ). The upper two histograms show the ONC derived from the X-ray COUP sample: the dashed is the 839 star COUP unobscured sample, and the solid histogram shows the addition of $82 K_{s}$-bright members undetected in X-rays. The middle dashed histogram is for the 321 star X-ray-unobscured Cep OB3b population obtained here. The lower dotted histogram is for the 64 star obscured Cep B population. The dash-dotted lines show three scaled versions of the ONC IMF (Muench et al. 2002), and the gray lines show three scaled versions of the Galactic field IMF (Kroupa 2002).

not so much interested in exact masses for individual objects as in the general trends of mass distributions and consistent treatment for comparison between clusters.

The IMF for the COUP 839 sample is shown as the upper dashed histogram in Figure 19. The COUP sample can be extended to account for incompleteness from instrumental causes: low local ACIS exposure time, high local background due to readout streaks from bright $\mathrm{ONC}$ sources, and contamination from the PSF wings of close bright X-ray sources. There are 82 such ONC stars with $A_{V} \lesssim 5$ mag and $K_{s}<13$ mag, corresponding to $M>0.1 M_{\odot}$ in Orion (Getman et al. 2005a). The resulting IMF of the $921(839+82)$ extended COUP ONC sample is shown as the upper solid histogram in Figure 19. Comparison with the ONC IMF fit based on KLFs obtained by Muench et al. (2002; dash-dotted line) indicates that the sample is complete down to $0.1 M_{\odot}$ and about $50 \%$ complete (120 vs. $\sim 240$ stars) within the low-mass stellar-substellar range of $0.03-0.1 M_{\odot}$. This incompleteness at low masses is an obvious consequence of an interaction between the observational flux limit and the X-ray/mass correlation (Preibisch et al. 2005).

We now turn to the Cep OB3b IMF derived in a similar manner. From 321 (64) PMS stars of the Chandra Cepheus unobscured (obscured) population, 310 (55) have masses estimated from NIR photometry (Table 2). Figure 20 confirms that the PMS stars in Cepheus (red, unobscured; blue, obscured) follow

TABLE 5

Integrated X-Ray Luminosities of Detected PMS Stars

\begin{tabular}{|c|c|c|c|c|c|}
\hline $\begin{array}{c}\text { Population } \\
\text { (1) }\end{array}$ & $\begin{array}{l}N_{\text {det }} \\
(2)\end{array}$ & $\sum_{\left(10^{32} \operatorname{ergs~s}^{-1}\right)}^{\sum L_{t}}$ & $\sum_{\left(10^{32} \operatorname{ergs~s}^{-1}\right)} L_{t, c}$ & $\sum_{\left(10^{32} \mathrm{ergs} \mathrm{s}^{-1}\right)}^{\sum L_{h}}$ & $\begin{array}{c}\sum_{\left(10^{32} \operatorname{ergs~s}_{h, c}^{-1}\right)} \\
(6)\end{array}$ \\
\hline Cep OB3b unobscured.......................... & 321 & $3.97 \pm 0.10$ & $7.38 \pm 0.89$ & $2.64 \pm 0.11$ & $2.82 \pm 0.14$ \\
\hline 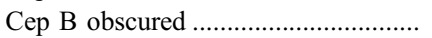 & 64 & $0.50 \pm 0.03$ & $2.68 \pm 1.49$ & $0.39 \pm 0.03$ & $0.52 \pm 0.05$ \\
\hline
\end{tabular}

Notes.-Col. (1): Type of Cepheus PMS population. Cols. (2)-(6): Number of PMS stars detected in X-rays in this Chandra observation and their total X-ray luminosities. 

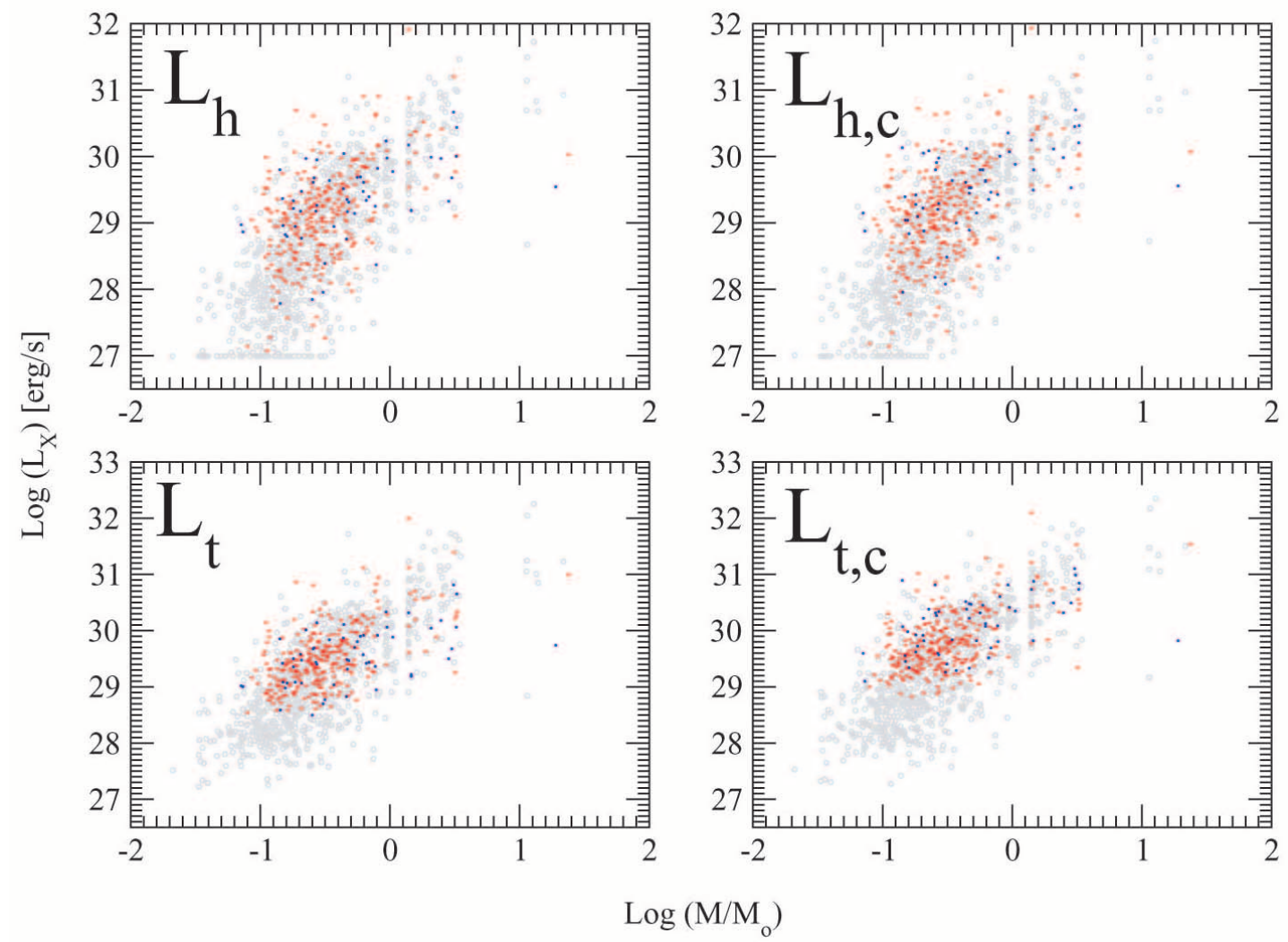

FIG. 20.-X-ray luminosity vs. stellar mass. The gray dots denote the COUP unobscured cool star sample of 839 stars. To be consistent with the current work, the plotted stellar masses for the COUP sample have been determined from an NIR color-magnitude diagram similarly to stellar masses derived for Cepheus stars. The red and blue dots represent the Cepheus unabsorbed and embedded populations, respectively. The X-ray luminosities are the same as in Fig. 12 .

$L_{\mathrm{X}}$ versus mass correlations very similar to that seen in COUP stars (gray dots). Even observed luminosity $L_{t}$, prone to changes as absorption varies, has a similar correlation with mass, as the median column densities for COUP and Cepheus unobscured populations are close to each other. Constant and slope parameters derived from the linear regression fit for the COUP unabsorbed sample are given for all four measures of X-ray luminosities in Table 6 . The regression parameters are slightly different from those derived in Preibisch et al. (2005) for the ONC lightly absorbed optical sample, but this can arise from the higher sensitivity of the COUP 839 sample than that of the optical sample, and from different methods used to estimate masses (Preibisch et al. 2005). For 11 (9) stars of the Cepheus unobscured (obscured) populations with unknown masses, we can use their measured X-ray luminosities with the regression fit parameters from Table 6 to get rough estimates on their masses

TABLE 6

Linear Regression Fits, $\log L_{\mathrm{X}}=A+B \log \left(M / M_{\odot}\right)$

\begin{tabular}{ccccc}
\hline $\begin{array}{l}L_{\mathrm{X}} \\
(1)\end{array}$ & $A$ & $\Delta A$ & $B$ & $\Delta B$ \\
& $(2)$ & $(3)$ & $(4)$ & $(5)$ \\
\hline & 29.95 & 0.08 & 2.25 & 0.13 \\
& 29.97 & 0.08 & 2.23 & 0.13 \\
\hline
\end{tabular}

Notes. - Col. (1): Type of $L_{\mathrm{X}}$. Cols. (2)-(3): Constant parameter of the linear regression and its standard deviation. Cols. (4)-(5): Slope of the linear regression and its standard deviation. and to add them to the appropriate bins of the IMF histograms. This is done in the lower histograms of Figure 19.

Although the histogram for the Cep OB3b population within the ACIS-I field (thick dashed line) is rough and clearly incomplete below $\sim 0.2 M_{\odot}$, we see a good agreement with the ONC IMF from 0.5 to $3 M_{\odot}$ but an excess of $\sim 20$ or more sources (depending on the choice of scaling the ONC-Muench IMF) in the $\log \left(M / M_{\odot}\right) \sim-0.5\left(0.3 M_{\odot}\right)$ bin. Expressed another way, the Cep OB3b IMF, in contrast to that of the ONC, does not exhibit the turnover from the Salpeter IMF around $0.5-0.6 M_{\odot}$.

\section{3. Сер OB3b KLFs}

We can estimate the total population of the Cepheus stellar clusters by extrapolating the IMFs below the incompleteness limit produced by the Chandra flux limit.

We obtain a coarse statistical sample of 2MASS PMS members of the Cep OB3b population in the ACIS-I field by subtracting a local background of foreground and background NIR objects from a control field of the 2MASS catalog. An IMF complete down to $M=0.08 M_{\odot}$ at a distance of 725 pc corresponds to $J \sim$ 16 mag, which is roughly the 2MASS completeness limit. Figure $13 c$ suggests that $\sim 90 \%$ of the Cep OB3b population is clustered within an area of $\sim 127 \mathrm{arcmin}^{2}$ lying within the ACIS-I field. This area roughly matches the pentagon shown in Figure 21 in which X-ray extended emission is seen $(\S 8)$. We extracted 2MASS sources from 30 control fields with $127 \operatorname{arcmin}^{2}$ area lying in a $3^{\circ} \times 3^{\circ}$ neighborhood of the Cep B cloud, as shown in Figure 22. It is not trivial to establish that the control fields are free from PMS stars, as the entire region is occupied by the members of the sparse stellar OB3 association and we trace a gradient in 


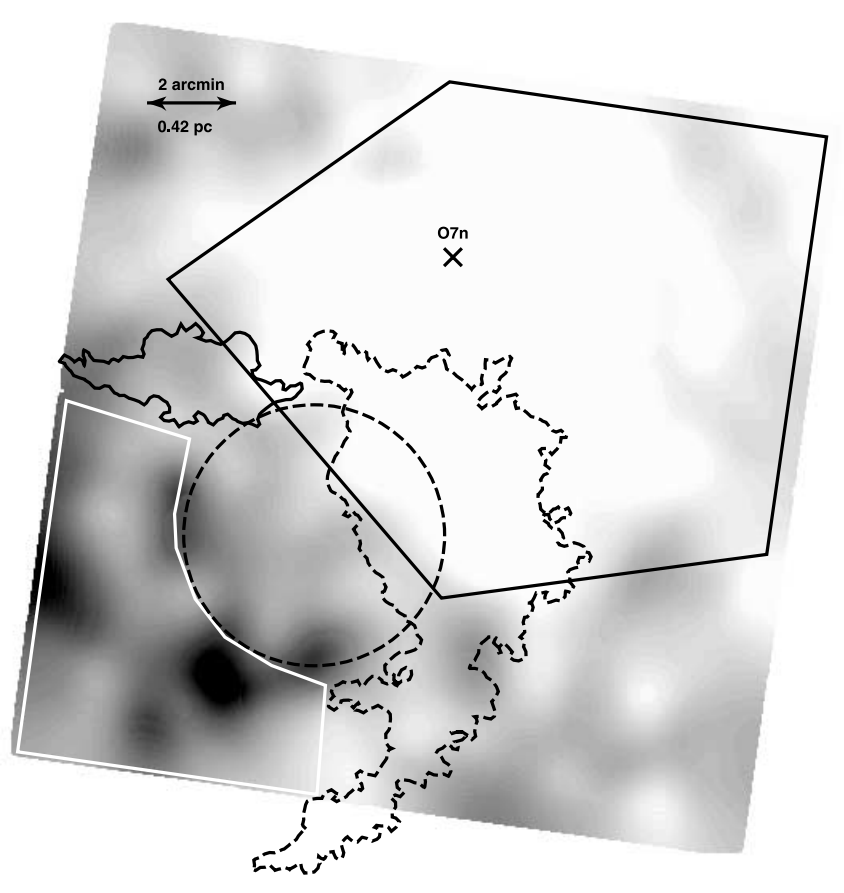

FIG. 21.-Soft-band (0.5-2 keV) Chandra image after removal of point sources with holes smoothed over using an adaptive smoothing algorithm. The pentagon indicates the extraction region for the diffuse X-ray emission, and the white line outlines the area used for background subtraction in spectral analysis. Dashed lines show the location of the $\mathrm{H}$ II region with ionization front and the Cepheus obscured population, and the cross marks the location of HD $217086(\mathrm{O} 7 \mathrm{n})$.

NIR source density as one progresses away from the cluster core. Toward the northwest, the extracted 2MASS source density clearly reflects the evolution of PMS source distribution in the Cep OB3 association - the number of sources systematically decreases down to 830 objects (at about $45^{\prime}$ [ $\left.\sim 9.5 \mathrm{pc}\right]$ away from the ACIS-I field), but then systematically rises again. The area with the least number of objects, 830 in a $127 \mathrm{arcmin}^{2}$ circle centered on $(\alpha, \delta)=\left(342^{\circ} .92539,+63^{\circ} .26147\right)$, perhaps lies at the boundary between different subgroups of the Cep OB3 association ("NW" background area); see Figure 1 of Sargent (1977). A second similar area is found toward the west, 814 2MASS sources at $\left(341^{\circ} .68270,+62^{\circ} .27262\right)$ as shown in Figure 22 ("W" background area). Figure 23 compares cumulative distributions of $J-H$ color excesses of 2 MASS sources seen toward the two control fields (dashed lines) with that seen toward the molecular cloud (dotted line). The large population of $J-H<1$ sources in the control fields supports the idea that these fields are relatively unobscured areas unaffected by the Cepheus molecular cloud.

To obtain a coarse IMF trend of the 2MASS unobscured sample of PMS stars within the ACIS-I field, we use the correlation $K_{S}=10.86-3.32 \log M$ based on the theoretical synthesized 1 Myr PMS model, Siess+Baraffe, employed here for $\left\langle A_{V}\right\rangle \sim$ $2.5 \mathrm{mag}\left(A_{K} \sim 0.28 \mathrm{mag}\right)$. This permits conversion of the KLF to stellar mass and production of two mass histograms (Fig. 24a, black solid lines) after subtraction of the two control fields and accounting for $10 \%$ of the unobscured Cep OB3b members dispersed outside the pentagon area.

The result is that our Chandra unobscured sample of 321 stars (Fig. 24a, dashed histogram) appears complete down to $\sim 0.4 M_{\odot}$, and that 400-450 stars are missing from the Chandra sample between 0.08 and $0.4 M_{\odot}$. Figure $24 a$ also compares this estimated IMF with that obtained for the ONC by Muench et al. (2002).

\subsection{Discussion}

Comparison of the IMF based on the 321 X-ray-detected PMS stars with the IMF based on the background-subtracted KLF suggests that the Chandra image is complete down to $\simeq 0.4 M_{\odot}$ (Fig. 24a). The difference between the infrared and X-ray source counts implies that 400-450 cluster stars are missing from the Chandra image, giving a total unobscured cluster population of $\sim 720-770$ stars in the $\sim 130-170 \mathrm{arcmin}^{2}$ area. We estimate the total X-ray luminosity of these undetected stars by multiplying the number of undetected stars by a typical X-ray luminosity for the appropriate mass bin using regression formulae from Table 6 . Table 7 summarizes results of this exercise and shows that a total of $\log L_{t} \sim 31.3$ and $\log L_{t, c} \sim 31.5 \mathrm{ergs} \mathrm{s}^{-1}$ is expected from the undetected population.

Assuming that the IMF for the embedded cluster also follows the mass distribution of the unabsorbed cluster, the embedded cluster of $64 \mathrm{X}$-ray-detected members is complete down to $M=$ $0.8 M_{\odot}$. We then estimate that 300-340 undetected obscured stars down to $M \sim 0.08 M_{\odot}$ are hidden in the cluster, giving a total of $\sim 360-400$ obscured stars.

We find that both the XLF and IMF comparisons indicate that the Cep OB3b population within the ACIS-I field exhibits, compared to the $\mathrm{ONC}$, more stars around $M \simeq 0.3 M_{\odot}$ relative to $0.5 M_{\odot}<M<3 M_{\odot}$ stars. This difference cannot be attributed to incompleteness in the COUP observation, as the addition of 82 stars missing from COUP does not significantly change the shape of COUP's IMF and hence XLF, and will not remove the discrepancy. It also cannot be attributed to incompleteness in the Cep OB3b observation (Fig. 18); inclusion of missing stars below $\log L_{t}=29.0 \mathrm{ergs} \mathrm{s}^{-1}$ would only increase the inferred number of lower mass cluster members. Similarly, the inclusion of binaries unresolved in Cep OB3b but resolved in the ONC (Getman et al. 2005a) would enhance the higher, not the lower, ends of the Cep OB3b XLF and IMF.

It is possible that our assumption of similar $(\sim 1 \mathrm{Myr})$ ages for all PMS stars of the Cep OB3b population within the ACIS-I field is incorrect. Recall that Pozzo et al. (2003) found the age of $\sim 1$ Myr for four out of six and $\gtrsim 3$ Myr for another two optical PMS candidates in our ACIS-I field. For the larger Cep OB3b association outside the ACIS-I field, the observed age spread in the $V$ versus $V-I$ color-magnitude diagram becomes even more dramatic, ranging from $\lesssim 1$ to $10 \mathrm{Myr}$, and is too broad to be accounted for by binarity, photometric uncertainty, and variability (Burningham et al. 2005). Subgroups of different ages may exist, each with different XLF and IMF characteristics. The XLFs are relatively insensitive to these age effects; between 1 and $10 \mathrm{Myr}$, only a slight decay (roughly 0.3 in $\log L_{t}$ ) is found in the COUP population (Preibisch \& Feigelson 2005). An older assumed age will have a bigger effect on the KLF. Figure $24 b$ summarizes a KLF analysis similar to that in $\S 7.3$, but assuming a cluster age of 3 Myr. In this case, X-ray-detected stars are more massive, with the completeness limit shifted from $\sim 0.4 M_{\odot}$ (for $1 \mathrm{Myr}$ ) down to $\sim 0.8 M_{\odot}$ (for $3 \mathrm{Myr}$ ). The excess of lower mass stars over the ONC IMF then mostly disappears. However, a younger age for Cep OB3b has two advantages: the unusual inferred IMF agrees with the unusual observed XLF ( $(7.1)$, which is insensitive to age assumptions, and the shape of the background-subtracted KLF more closely agrees with the IMF of the X-ray-detected population for 1 Myr compared to $3 \mathrm{Myr}$ (Fig. 24). An argument in favor of the older age can be seen in Figure 24b: the backgroundsubtracted KLF more closely matches the Galactic field IMF ( gray line) than in the case of $1 \mathrm{Myr}$. 


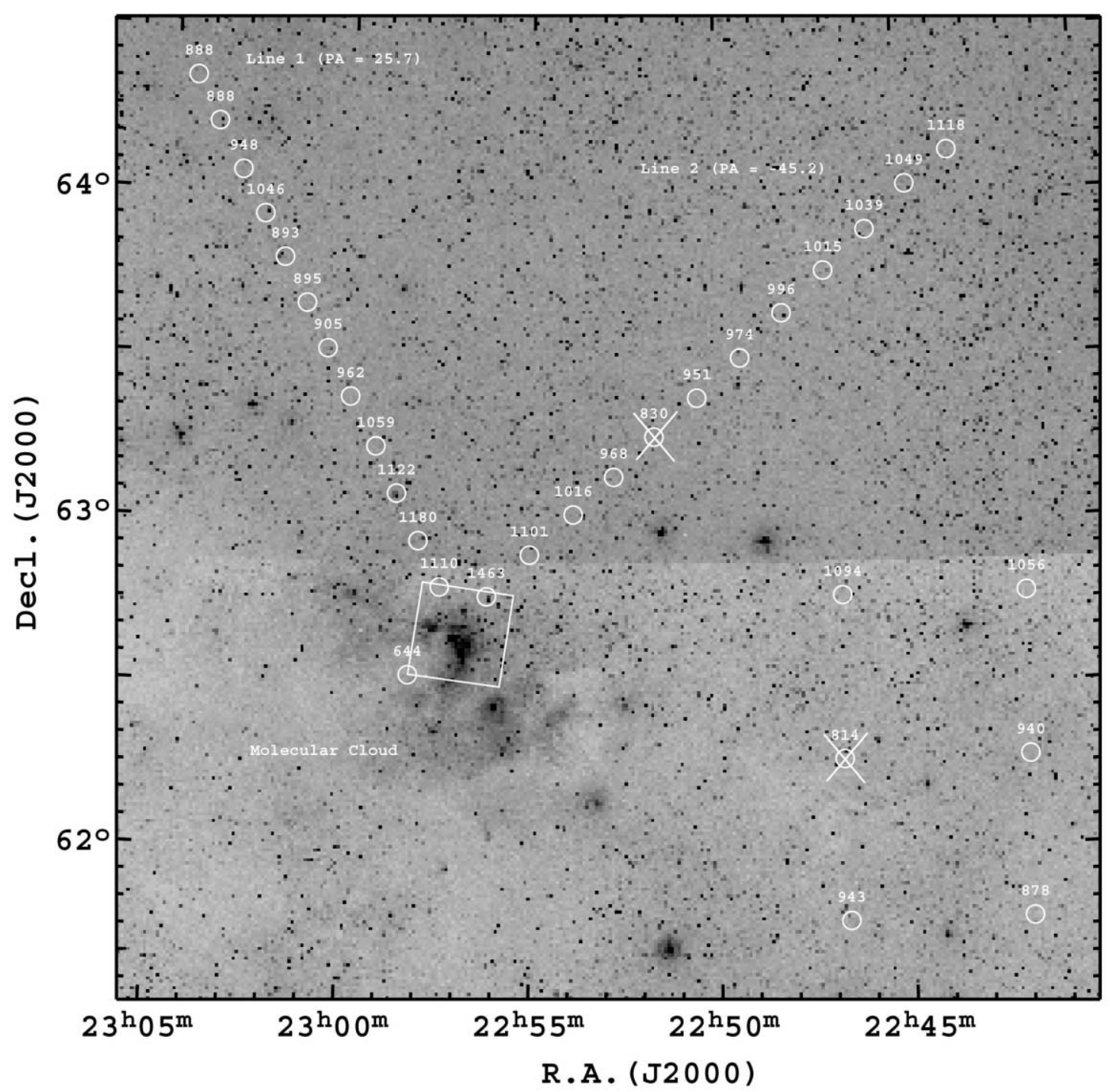

FIG. 22.-Background regions used in estimation of the Cep OB3b unobscured cluster KLF. This is a $3^{\circ} \times 3^{\circ}$ image from the $R$-band DSS. The ACIS-I field is outlined by the white square. The local number of 2MASS sources in a $127 \mathrm{arcmin}^{2}$ region was measured at each of the circled locations. The two areas that we use for the background subtraction, with 814 sources in the western field and 830 sources in the northwestern field, are marked by white crosses.

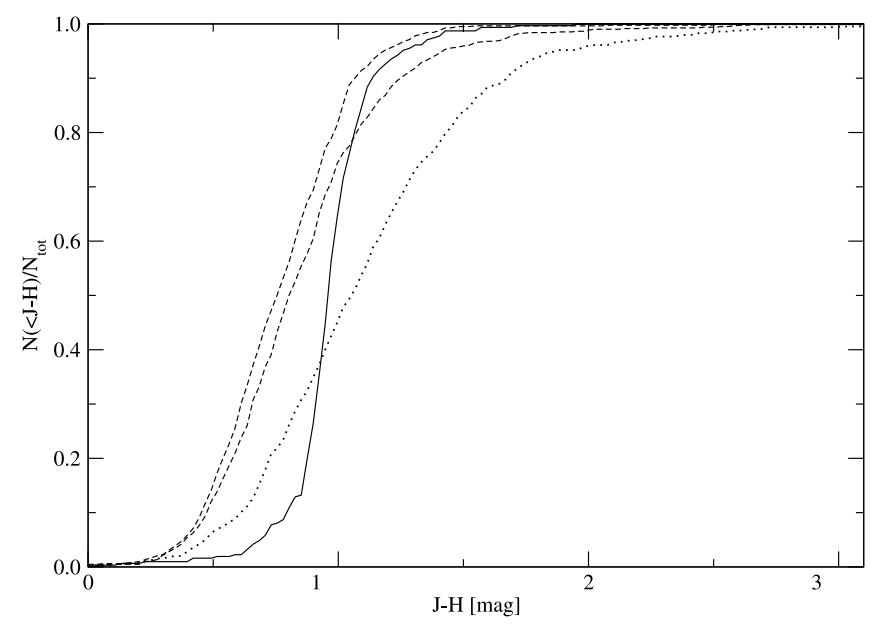

Fig. 23.-Comparison of 2MASS $J-H$ color distributions in the cluster, molecular cloud, and background fields. The solid line shows PMS stars of the Cep OB3b unobscured population in the ACIS-I field. The dotted line shows absorbed 2MASS stars in the molecular cloud (region around $22^{\mathrm{h}} 58^{\mathrm{m}},+62^{\circ} 30^{\prime}$ in Fig. 22). The upper (lower) dashed lines show 2MASS sources in the western (northwestern) background fields.
We thus emerge with a clear determination that the XLFs of Cep OB $3 \mathrm{~b}$ and the ONC differ, but without a clear interpretation of this effect. It may be due to a different IMF with more lower mass stars in Cep OB3b, or to differences in age and history of star formation in the two clusters. Follow-up optical photometry and spectroscopy of $321 \mathrm{Cep}$ OB3b X-ray sources would assist in unraveling these possibilities, as individual (rather than statistical) masses and ages can be directly estimated on the HertzsprungRussell diagram.

If we assume that the $1 \mathrm{Myr}$ ages are correct, then the inferred IMF difference may be related to differences between the physical processes of star formation in the Cep B and Orion molecular clouds. The average Jeans masses may be lower in Cep B than in Orion due to different densities or pressures. However, if the Jeans mass is universal around $M_{\mathrm{J}} \sim 0.1 M_{\odot}$ (Elmegreen 1999), then the IMF difference could arise from the prevalence of different star formation mechanisms in the two clouds (Elmegreen 2004). In this multicomponent scenario, lower mass stars would have formed similarly in Cep B and Orion from prestellar condensations of $M_{\mathrm{J}}$ mass, but higher mass stars would have grown from coalescence of prestellar condensations 


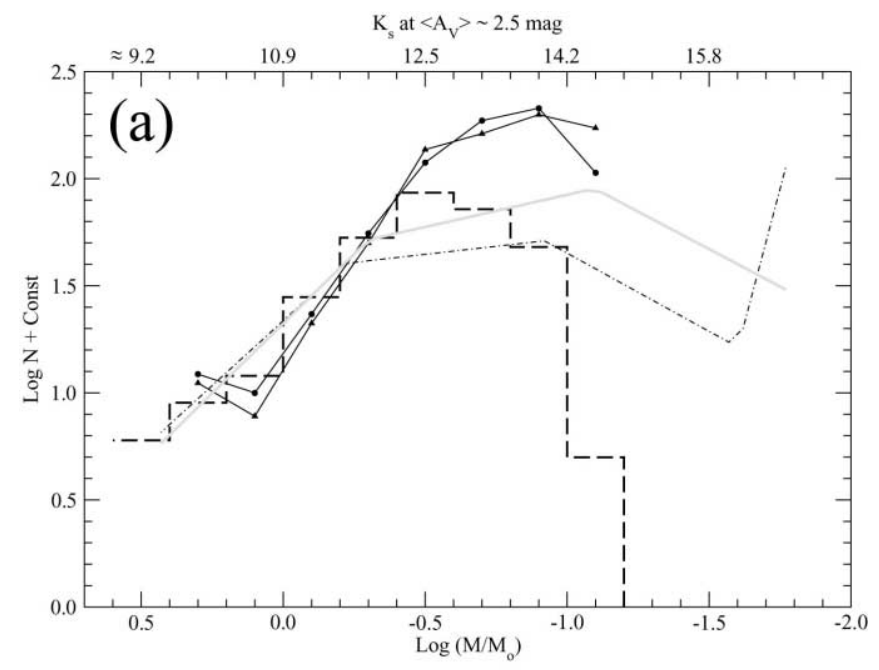

TABLE 7

InTEgrated X-Ray Luminosities of Undetected Unobscured PMS Stars

\begin{tabular}{|c|c|c|c|c|c|c|}
\hline \multirow[b]{2}{*}{$\begin{array}{c}\log \text { Mass BIN } \\
\text { (1) }\end{array}$} & \multicolumn{2}{|c|}{$N_{\text {undet }}$} & \multicolumn{2}{|c|}{$\sum L_{t}\left(10^{30} \mathrm{ergs} \mathrm{s}^{-1}\right)$} & \multicolumn{2}{|c|}{$\sum L_{t, c}\left(10^{30} \operatorname{ergs~s}^{-1}\right)$} \\
\hline & $\begin{array}{l}\text { NW } \\
(2)\end{array}$ & $\begin{array}{l}\text { W } \\
(3)\end{array}$ & $\begin{array}{l}\text { NW } \\
(4)\end{array}$ & $\begin{array}{l}\text { W } \\
(5)\end{array}$ & $\begin{array}{l}\mathrm{NW} \\
(6)\end{array}$ & $\begin{array}{l}\text { W } \\
\text { (7) }\end{array}$ \\
\hline$-0.5 \ldots \ldots \ldots \ldots \ldots$ & 32 & 50 & 5.62 & 8.79 & 8.71 & 13.61 \\
\hline$-0.7 \ldots \ldots \ldots \ldots \ldots$ & 115 & 90 & 8.08 & 6.33 & 12.52 & 9.80 \\
\hline$-0.9 \ldots \ldots \ldots \ldots$ & 165 & 151 & 4.64 & 4.25 & 7.18 & 6.58 \\
\hline$-1.1 \ldots \ldots \ldots \ldots \ldots$ & 102 & 167 & 1.15 & 1.88 & 1.78 & 2.91 \\
\hline Total .............. & 414 & 458 & 19.49 & 21.24 & 30.20 & 32.90 \\
\hline
\end{tabular}

Notes.-Col. (1): Logarithm of mass bin. Cols. (2)-(3): Estimated number of X-ray-undetected stars for subtractions of "NW" and "W" local backgrounds. Cols. (4)-(7): Expected observed and corrected-for-absorption X-ray luminosities.

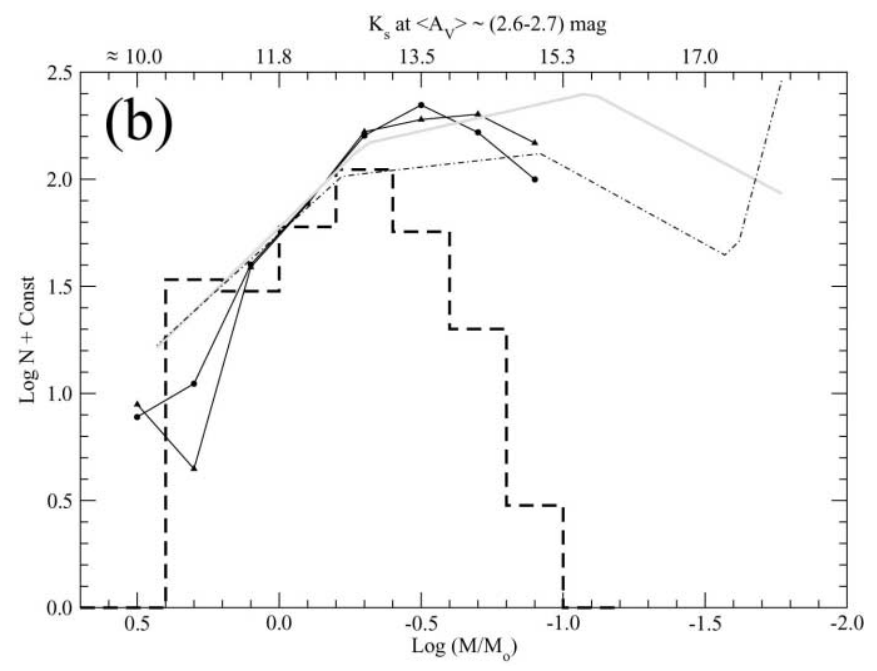

on the molecular cloud in the southeastern corner of the field, as shown in Figure 21. The resulting spectrum of the diffuse emission is shown in Figure 25a. It can be reasonably well fit by a single-temperature optically thin thermal plasma with $k T \sim$ $0.8 \mathrm{keV}$ subject to $\log N_{\mathrm{H}} \sim 21.7 \mathrm{~cm}^{-2}\left(A_{V} \sim 2.5 \mathrm{mag}\right)$ absorption, which is typical for the unobscured Cepheus region. The observed (absorption corrected) X-ray luminosity of the diffuse emission is $\log L_{t} \simeq 31.3\left(\log L_{t, c} \simeq 31.8\right) \mathrm{ergs} \mathrm{s}^{-1}$.

This is substantially weaker than the $\log L_{t} \simeq 32-33 \mathrm{ergs} \mathrm{s}^{-1}$ in diffuse $\mathrm{X}$-rays attributed to colliding $\mathrm{O}$ star winds in other $\mathrm{H}$ II regions such as M17 (Townsley et al. 2003). In theory, a strong wind from a single massive star, interacting with the surrounding ISM, may fill the surrounding volume with hot shocked gas and produce X-ray emission as high as $10^{35}-10^{36} \mathrm{ergs} \mathrm{s}^{-1}$. The HD $217086 \mathrm{O} 7 \mathrm{n}$ star has a high wind terminal velocity of $V_{\infty}=$ $2510 \mathrm{~km} \mathrm{~s}^{-1}$ derived from ultraviolet spectroscopy by Prinja et al. (1990). Such a fast wind may heat the gas to temperatures of $10^{7}-10^{8} \mathrm{~K}$. On the other hand, HD 217086 has a low massloss rate of $\dot{M} \sim 2 \times 10^{-7} M_{\odot} \mathrm{yr}^{-1}$ (Howarth \& Prinja 1989). Stevens \& Hartwell (2003) have calculated a scaling between expected X-ray emission and the wind scaling factor $X_{\mathrm{cl}}=$ $\dot{M}^{2} /\left(R_{c} V_{\infty}\right)$ at temperatures higher than $10^{7} \mathrm{~K}$. For a cluster core radius of $R_{c} \sim 1 \mathrm{pc}$ and the HD 217086 wind parameters, the predicted diffuse X-ray luminosity is $\log L_{\mathrm{X}}<28 \mathrm{ergs} \mathrm{s}^{-1}$ for the wide range $0.1-1$ of the thermalization efficiency parameter $\eta$. This is at least 3 orders of magnitude smaller than the X-ray luminosity derived for the detected diffuse X-ray emission (Table 8). This casts considerable doubt on the idea that the $\mathrm{O}$ star wind shocks are responsible for the emission.

The alternative and more natural scenario for the origin of the diffuse emission in Cepheus is X-ray emission from the 400 450 lower mass PMS stars, which are not individually detected (§ 7.3) due to the high sensitivity limits ( $\log L_{t, c, \lim } \simeq 29$ for the lightly obscured population; Fig. 17) of the current Chandra observation. Recall that the Galactic background contribution to the diffuse emission is small, $\log L_{t} \lesssim 30$, as indicated by the simulations described in $\S 5.3$. Several arguments support the explanation for the diffuse emission from an unresolved PMS component in Cepheus.

1. The diffuse emission spatially coincides with the clustered $\mathrm{X}$-ray-detected members of the lightly obscured Cep OB3b population as expected if cluster stars are responsible. Compare the distribution of detected stars in Figure 13 with the diffuse emission in Figure 21.

2. The spectral shape of the diffuse emission in Cepheus closely resembles the composite spectrum of the detected X-ray

\footnotetext{
${ }^{14}$ Details on the production of such images and the extraction of diffusesource and background spectra can be found online in the recipes for $\mathrm{AE}$ at T.//www.astro.psu.edu/xray/docs/TARA/ae_users_guide, § 7.1.2. They have previously applied to Chandra images of high-mass star formation re method.
} 

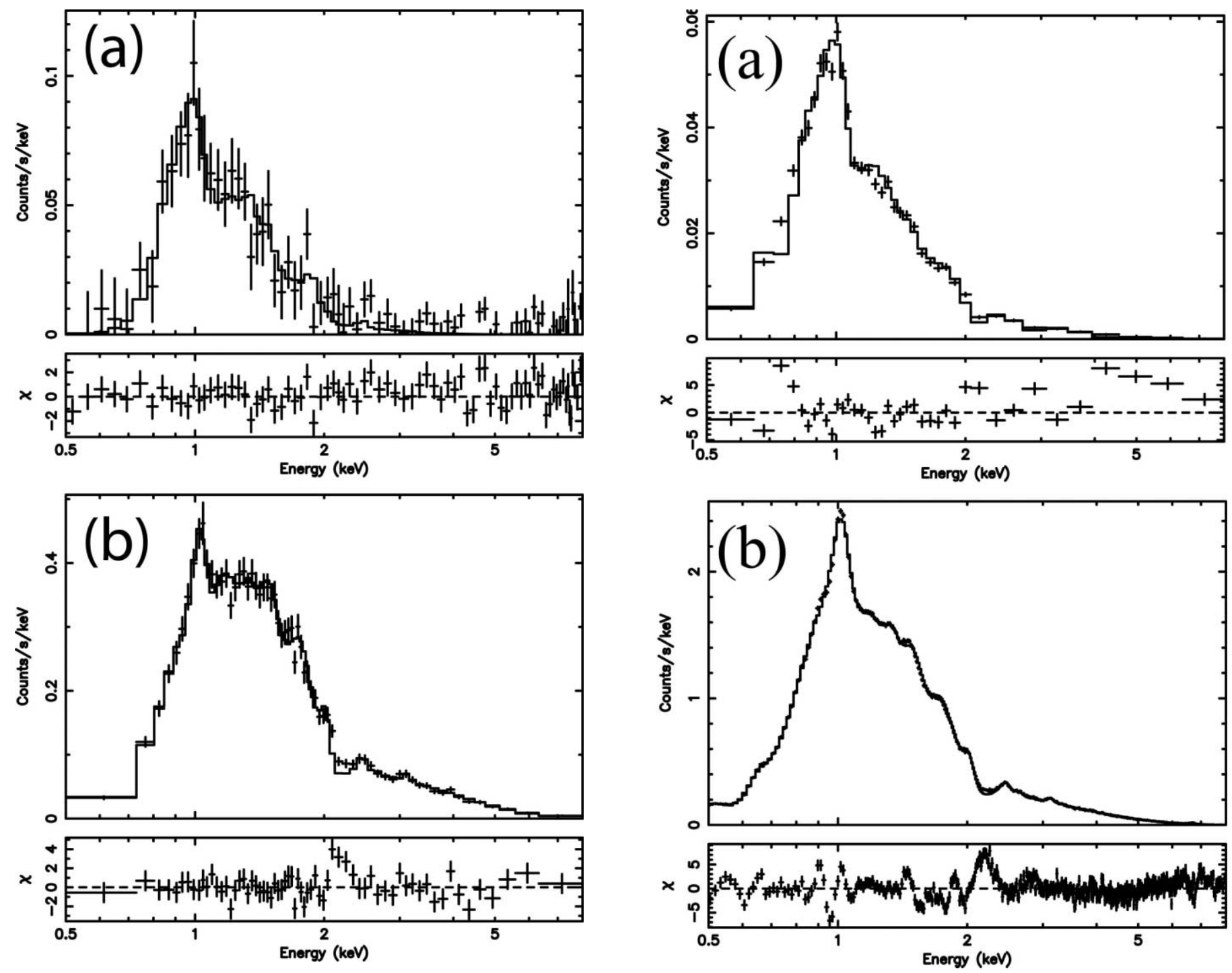

FIG. 25.-Cepheus composite X-ray spectra. (a) Spectrum of the diffuse component detected in the $127 \mathrm{arcmin}^{2}$ region shown in Fig. 21 ( points with error bars) with spectral model (histogram). (b) The composite spectrum and spectral model for 321 PMS stars of the unobscured X-ray population. The lower plots show residuals between the data and models in units of $\chi^{2}$.

population as shown in Figure 25 for the Cepheus observation. A similar finding can be seen in the cool, lightly obscured COUP population (839 sources introduced in $\S 7.1$ ). Figure 26 illustrates the resemblance between a COUP subsample containing the weakest ( $\log L_{t, c}<29$ ) sources (Fig. 26a), corresponding to

FIG. 26.-Composite X-ray spectra of the cool unobscured ONC population. (a) Subsample of 326 weak $\left(\log L_{t, c}<29\right)$ sources. (b) Full sample of 820 sources with no pileup.

the Cepheus diffuse emission here attributed to the undetected population, and the full COUP sample ( 820 sources, excluding those piled up; Fig. 26b), corresponding to the detected Cepheus population. The plasma temperature of $k T \sim 0.8 \mathrm{keV}$ is similar for both the Cepheus diffuse emission and the COUP weak sources (Table 8). This is not a surprise, as it has been established from the COUP project that the temperature of the hot plasma

TABLE 8

X-Ray Properties of the Diffuse Component and PMS Populations

\begin{tabular}{|c|c|c|c|c|c|c|}
\hline $\begin{array}{l}\text { Object } \\
\text { (1) }\end{array}$ & $\begin{array}{c}\text { Count Rate } \\
\text { (counts } \mathrm{ks}^{-1} \text { ) } \\
\text { (2) }\end{array}$ & $\begin{array}{c}N_{\mathrm{H}} \\
\left(10^{22} \mathrm{~cm}^{-2}\right) \\
(3)\end{array}$ & $\begin{array}{c}k T_{1} \\
(\mathrm{keV}) \\
(4)\end{array}$ & $\begin{array}{c}k T_{2} \\
(\mathrm{keV}) \\
(5)\end{array}$ & $\begin{array}{c}L_{t} \\
\left(10^{31} \mathrm{ergs} \mathrm{s}^{-1}\right) \\
(6)\end{array}$ & $\begin{array}{c}L_{t, c} \\
\left(10^{31} \mathrm{ergs} \mathrm{s}^{-1}\right) \\
(7)\end{array}$ \\
\hline Cepheus point sources & 570.0 & $0.33 \pm 0.23$ & $0.61 \pm 0.23$ & $4.51 \pm 0.62$ & $54.34 \pm 4.41$ & $76.74 \pm 19.91$ \\
\hline 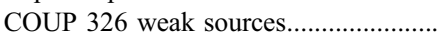 & 43.1 & $0.34 \pm 0.15$ & $0.77 \pm 0.18$ & $\ldots$ & $1.93 \pm 0.06$ & $4.84 \pm 2.10$ \\
\hline
\end{tabular}

Notes.-Col. (1): Defines object: either Cepheus X-ray diffuse emission, or 321 X-ray-detected point source members of the Cep OB3b unobscured population, or 326 weak $\left(\log L_{t, c}<29\right)$ members of the COUP unobscured population. Col. (2): Net count rate. Col. (3): Column density inferred from XSPEC fits, using the wabs (apec) model for the diffuse emission and wabs (vapec+vapec) for the point-source population. Cols. (4)-(5): Plasma temperatures inferred from XSPEC fits. Cols. (6)-(7): Observed and absorption-corrected X-ray luminosities in the $0.5-8.0 \mathrm{keV}$ band, inferred from the X-ray spectral fits. 
component for ONC T Tauri stars having two-temperature spectral fits decreases as X-ray luminosity drops, while the cooler plasma component remains around $10 \mathrm{MK}(\sim 0.86 \mathrm{keV})$ in nearly all T Tauri stars (Preibisch et al. 2005).

3. The inferred integrated (absorption corrected) X-ray luminosity of the missing PMS population of the unobscured Cep OB3b cluster, estimated in $\S 7$ to be $\log L_{t} \simeq 31.3\left(\log L_{t, c} \simeq\right.$ 31.5 ) ergs $\mathrm{s}^{-1}$, is very close to the observed diffuse X-ray luminosity of $\log L_{t} \simeq 31.3\left(\log L_{t, c} \simeq 31.8\right) \mathrm{ergs} \mathrm{s}^{-1}$. $^{15}$

A strong $\mathrm{Ne}$ IX and $\mathrm{Ne} x$ line complex is present in the composite spectrum of the Cep OB3b unobscured PMS members (Fig. 25b). This emission line is prominent in the composite spectrum of COUP ONC stars (Fig. 26b), and Feigelson et al. (2005) have argued that this line should be a useful diagnostic of magnetically active PMS stars. ${ }^{16}$

\section{TWO INTERESTING X-RAY STARS}

\subsection{Wind Shocks in the O7n Star HD 217086}

Among the members of the Cep OB3b population within the ACIS-I field, only one massive star is seen in X-rays: the O7n star HD 217086, Chandra source 240. It is thought to be the principal ionizing source of the $\mathrm{S} 155 \mathrm{H}$ II region.

Our understanding of the production of X-rays in the inner winds of O stars is somewhat confused. Some stars show the soft, constant X-ray emission expected from myriad small shocks in unstable radiatively accelerated winds (Lucy \& White 1980). But others show hard spectral components, rotational modulations, flaring light curves, and unexpected spectral line shapes that indicate other processes are operative. Stelzer et al. (2005) performed an in-depth study of the X-ray emission of the early-type stars of the ONC and found that only two of nine hot massive stars with spectral class O7-B3 exhibit exclusively the constant soft spectrum at $\log \left(L_{\mathrm{X}} / L_{\text {bol }}\right) \sim-7$ expected from the standard model involving small-scale shocks from unstable radiatively accelerated winds. Most of the other massive stars, including the brightest, $\theta^{1}$ Ori $\mathrm{C}(\mathrm{O} 7)$, show combinations of soft wind emission and other phenomena that could be explained by a magnetically confined wind shock model, where the harder and variable X-rays are produced by magnetically mediated large-scale shocks (Babel \& Montmerle 1997; ud-Doula \& Owocki 2002).

Although Chandra source 240 falls by chance on an ACIS CCD chip gap, this should have little effects on its properties once we evaluate the exposure map value at the source's location. Its

\footnotetext{
15 The difference of a factor of $\sim 2$ between absorption-corrected X-ray luminosities of $\log L_{t, c} \simeq 31.5$ expected from faint cluster stars and $31.8 \mathrm{ergs} \mathrm{s}^{-1}$ observed in diffuse emission can be explained as a statistical artifact. The same discrepancy is seen for the integrated absorption-corrected luminosities of 326 COUP weak sources considered individually in comparison with the absorption-corrected luminosity derived from their composite spectrum. When considered individually, the poor statistics of these sources lead to successful fits by a single temperature with median plasma energy $k T \sim 1.6 \mathrm{keV}$. This is noticeably harder than the $k T \simeq$ $0.8 \mathrm{keV}$ energy derived from the combined fit. This arises because of a bias toward detecting photons at energies where the Chandra ACIS effective area is greatest around $1.5 \mathrm{keV}$; substantially softer or harder photons are not detected in sufficient quantity in individual weak sources to be represented in the XSPEC fitting procedure. Due to this bias, absorption-corrected X-ray luminosities $L_{t, c}$ of weak sources may often be underestimated due to the unseen soft temperature component, as discussed by Getman et al. (2005b) and Feigelson et al. (2005).

16 One may note strong residuals between the spectral model and data around $2.1 \mathrm{keV}$ in Figs. $25 b$ and $26 b$. These are likely instrumental features related to the fact the effective area of the Chandra telescope mirrors is not properly calibrated at the iridium $\mathrm{M}$ edge near $2.1 \mathrm{keV}$, possibly due to a thin contaminant layer on the mirrors. For more details see http://cxc.harvard.edu/ccw/proceedings/04_proc/ presentations/jerius.
}
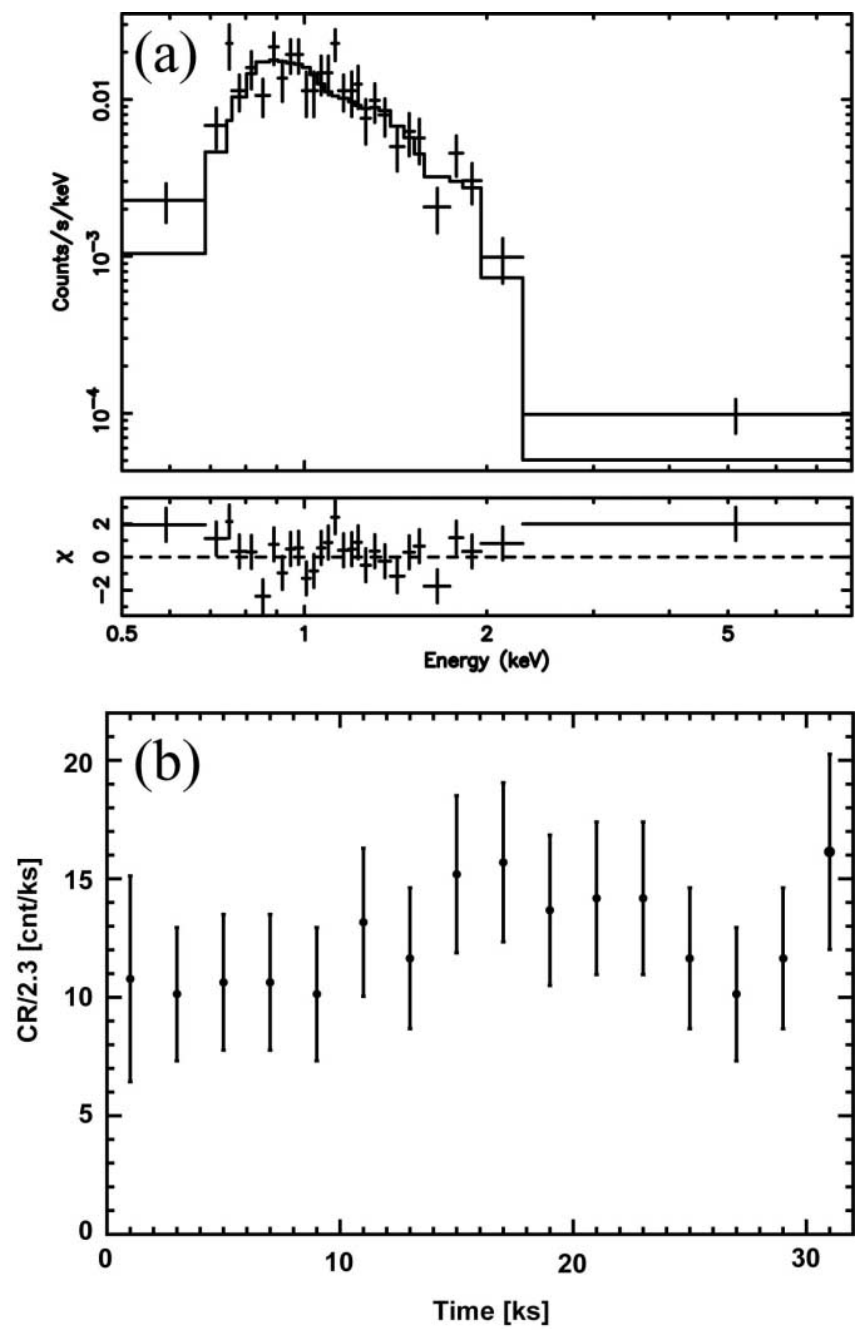

FIG. 27.-X-ray properties of the O7n star HD 217086 (Chandra source 240): (a) ACIS spectrum, and (b) light curve.

effective exposure is $12.9 \mathrm{ks}$ (Table 1), about 2.3 times shorter than the $30.1 \mathrm{ks}$ effective exposure for the whole observation. The source spectrum and light curve are shown in Figure 27. To ensure that the interaction of satellite dithering with the chip gap does not affect the shape of the light curve, the light curve is shown with $2 \mathrm{ks}$ bin size, which is 2 times larger than the dithering period. A Kolmogorov-Smirnov test shows only marginal evidence $\left(P_{\mathrm{KS}} \sim\right.$ $0.07)$ for variability during the $8 \mathrm{hr}$ observation; it was similarly constant during the earlier ROSAT observation (Naylor \& Fabian 1999). The X-ray spectral analysis gives a count rate of $\sim 32.3$ counts $\mathrm{ks}^{-1}$, plasma temperature of $\sim 0.6 \mathrm{keV}$, and X-ray luminosity in the $0.5-8.0 \mathrm{keV}$ band of $\log L_{t, c} \sim 31.5 \mathrm{ergs} \mathrm{s}^{-1}$, after correction for $\log N_{\mathrm{H}} \simeq 21.7 \mathrm{~cm}^{-2}$ absorption. Comparison with the coarse bolometric luminosity derived from the 2MASS color-magnitude diagram ( Table 2) gives $\log \left(L_{t, c} / L_{\mathrm{bol}}\right)=-7$ with a stellar mass of $\sim 24 M_{\odot}$. Thus its soft constant X-ray emission at the $\log \left(L_{t, c} / L_{\mathrm{bol}}\right)=-7$ level is probably attributable to the traditional Lucy-White model of small-scale wind shocks, without invoking magnetic fields.

\subsection{Superflare in Chandra Star 294}

The brightest X-ray source in the ACIS field is source 294, with 2938 counts extracted from the $89 \%$ encircled energy region. Source 294 has an extraordinarily luminous and hard X-ray flare whose amplitude, power, and temperature are similar to the 

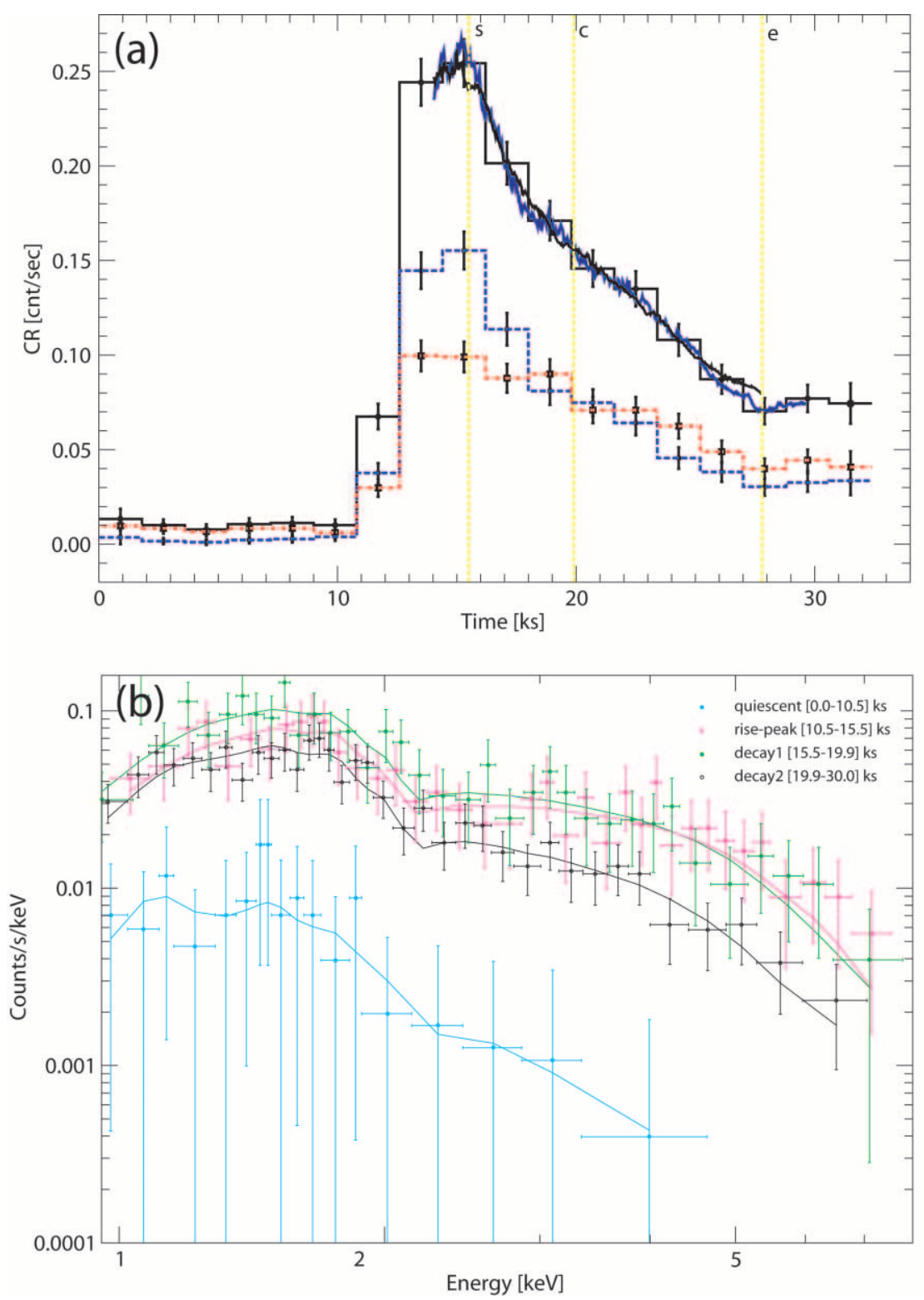

FIG. 28.-X-ray properties of the superflare in Chandra source 294. (a) Light-curve histograms of source 294 with a bin size of half an hour for the full energy band $(0.5-8.0 \mathrm{keV}$; black histogram), soft band $(0.5-2.0 \mathrm{keV}$; red histogram), and hard band $(2.0-8.0 \mathrm{keV}$; blue histogram). Black and blue curves represent unbinned but adaptively smoothed versions of the light curve with a window size of at least 256 and 570 counts, respectively. Vertical labeled yellow lines give two characteristic decay ranges, $[s, c]=[15.5,19.9 \mathrm{ks}]$ and $[c, e]=[19.9,27.8 \mathrm{ks}]$, found from our further analysis of the adaptively smoothed median energies (Fig. 31 ). (b) Spectra of four phases of the light curve with single-temperature optically thin thermal plasma fits, subject to $\log N_{\mathrm{H}} \sim 21.8 \mathrm{~cm}^{-2}$ absorption: quiescent level (cyan; $k T \sim 1.7 \mathrm{keV}$ ), flare rise and peak (magenta; $k T \sim 64 \mathrm{keV}$ ), early decay phase (green; $k T \sim 12 \mathrm{keV}$ ), and late decay phase (black; $k T \sim 4.5 \mathrm{keV}$ ).

brightest flares seen in the Orion population (Grosso et al. 2004; Favata et al. 2005). Its light curve has the typical morphology of stellar flares with a fast rise and gradual decay (Fig. 28a). During the $\sim 1.1 \mathrm{hr}$ rise phase the count rate rose by factors of $\sim 20, \sim 50$, and $\sim 10$ in the full, hard, and soft bands, respectively. Due to the short exposure of our observation, only the first $\sim 4.4 \mathrm{hr}$ of the decay phase were observed. We divide this phase into a rapid and a slow cooling phase.

Spectra extracted from the quiescent, rise and peak, rapid cooling, and slow cooling phases are compared in Figure $28 b$. They all can be successfully represented by single-temperature optically thin plasma models seen through a similar amount of gas absorption of $\log N_{\mathrm{H}}=21.8 \mathrm{~cm}^{-2}$. During the quiescent phase, the plasma had an average temperature of $\sim 1.7 \mathrm{keV}(\sim 20 \mathrm{MK})$ that heated rapidly during the flare rise phase of $\sim 5 \mathrm{ks}$ to $\langle k T\rangle \gg$
$15 \mathrm{keV}$. The nominal XSPEC fit gives $64 \mathrm{keV}(\sim 740 \mathrm{MK})$. Over the next $\sim 4.4 \mathrm{ks}$, the plasma rapidly cooled down to $\langle k T\rangle \sim$ $12 \mathrm{keV}(\sim 140 \mathrm{MK})$, and cooling continued during the slow cooling phase to $\langle k T\rangle \sim 4.5 \mathrm{keV}(\sim 50 \mathrm{MK})$ over the last $10 \mathrm{ks}$. $\mathrm{X}$-ray luminosities rose from a quiescent level of $L_{t, c}=9.7 \times$ $10^{30}\left(L_{h, c}=3.5 \times 10^{30}\right) \mathrm{ergs} \mathrm{s}^{-1}$ in the full (hard) energy bands to $L_{t, c}=2.2 \times 10^{32}\left(L_{h, c}=1.8 \times 10^{32}\right) \mathrm{ergs} \mathrm{s}^{-1}$ during the risepeak phase. These temperatures and luminosities are similar to the most luminous X-ray flares ever recorded in young stellar objects: V773 Tau in the Taurus-Auriga clouds with $L_{\mathrm{pk}} \gtrsim 10^{33} \mathrm{ergs} \mathrm{s}^{-1}$ and $T_{\mathrm{pk}} \gtrsim 100 \mathrm{MK}$ (Tsuboi et al. 1998), LkH $\alpha 312$ in the M78 reflection nebula of Orion with $L_{\mathrm{pk}} \simeq 10^{32} \mathrm{ergs} \mathrm{s}^{-1}$ and $T_{\mathrm{pk}} \simeq$ $90 \mathrm{MK}$ (Grosso et al. 2004), and COUP 1568 in the Orion Nebula cluster with $L_{\mathrm{pk}} \simeq 7.8 \times 10^{32} \mathrm{ergs} \mathrm{s}^{-1}$ and $T_{\mathrm{pk}} \sim 500 \mathrm{MK}$ (Favata et al. 2005). 


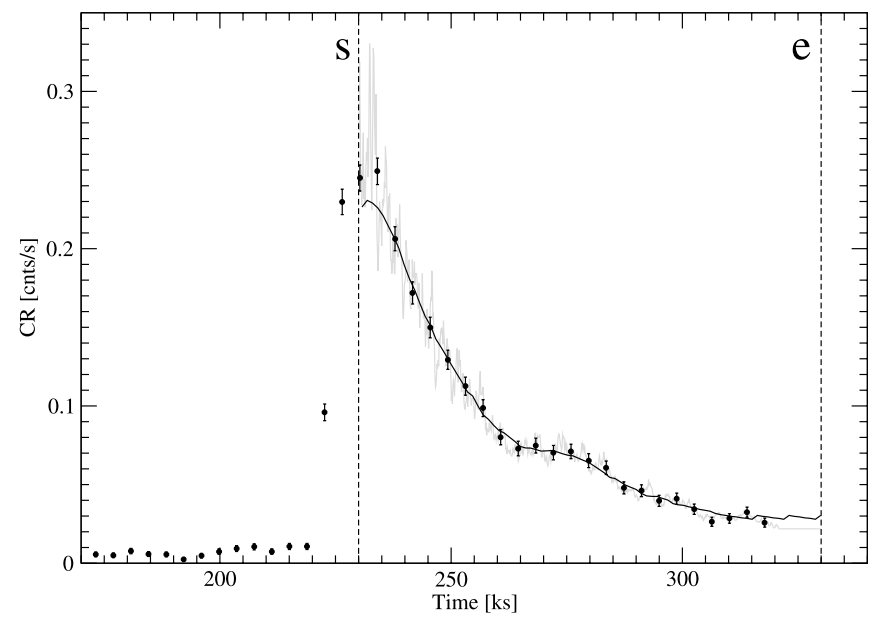

FIG. 29.-Light-curve histogram (black dots with uncertainties) with a bin size of $1.06 \mathrm{hr}$ for the COUP source 1343, used here to validate our method of modeling the Cepheus source 294 flare. Gray and black lines present adaptively smoothed versions of the light curve with a window size of at least 100 and 625 counts, respectively. Vertical labeled dashed lines give the time range $[\mathrm{s}, \mathrm{e}]=[230,330 \mathrm{ks}]$ over which our analysis of the adaptively smoothed median energies was performed.

With detailed data on the decay of flux and temperature during the cooling phase, we can infer properties of the radiating plasma structures within the framework of the time-dependent hydrodynamic model in a coronal magnetic loop. Reale et al. (1997) establish a formula for estimating loop size with a proper account of the possibility of prolonged heating during the decay phase. They find $L \propto \tau_{\mathrm{lc}}\left(T_{\mathrm{pk}}\right)^{1 / 2} / F(\zeta)$, where $L$ is the length of the loop, $\tau_{\mathrm{lc}}$ is the flare decay time, and $T_{\mathrm{pk}}$ is the plasma temperature at the peak. The quantity $F(\zeta)$ is a correction factor for prolonged heating that is a function of the slope $\zeta$ of the trajectory in the temperature-density diagram; $F(\zeta)$ must be calibrated for each $\mathrm{X}$-ray telescope and detector. The model assumes that the plasma is confined within the semicircular loop of uniform cross section, whose geometry remains unaltered during the whole flare, and that the motion and energy transport occur along magnetic field lines of the loop. Favata et al. (2005) applied this approach to infer the size of the flaring structures for bright $\mathrm{X}$-ray flares in the Orion Nebula cluster population with $F(\zeta)$ calibrated to Chandra and ACIS-I. They found several superflares with inferred loop sizes long enough to link the stellar photosphere with the inner rim of the circumstellar disk.

The slope $\zeta$ is usually measured in the $\log T$ versus $\log (\mathrm{EM})^{1 / 2}$ plane, where $(\mathrm{EM})^{1 / 2}$ is used as a proxy for the plasma density. In the case of the source 294 flare, we adopt a slightly different technical approach. Instead of performing time-sliced spectroscopy with XSPEC over a few characteristic time blocks of the light curve as shown in Figure 28b, we employ an adaptively smoothed median energy time series to derive hundreds of time points of $(k T, \mathrm{EM})$ along the decay phase of the light curve. This is achieved by calibrating median energies to temperatures using the spectral simulations described in $\S 4$. Median energies are more statistically stable for small- $N$ data sets than $k T$ parameters of nonlinear XSPEC regressions. We assume that $N_{\mathrm{H}}$ is constant, and we ignore the weak soft spectral component that is likely present from the quiescent background.

We test this method on the decay phase of the flare in the Orion COUP source 1343 studied in detail by Favata et al. (2005). The light curve for the COUP source 1343 is shown in Figure 29 with black dots representing the light curve histogram, binned with a bin size of $\sim 1 \mathrm{hr}$. The peak-decay phase studied in our analysis ranges from 230 to $330 \mathrm{ks}$ from the beginning of the COUP observations. The black and gray lines represent smoothed versions of the light curve using the adaptively smoothed algorithm from AE with different minimum numbers of counts in the kernel (625 and 100 count minimum, respectively). The 100 count kernel is too small as it leaves many noise spikes, so we use the 625 count kernel, although it has oversmoothed the narrow peak phase of the light curve. Results of the analysis appear in Figure 30. The
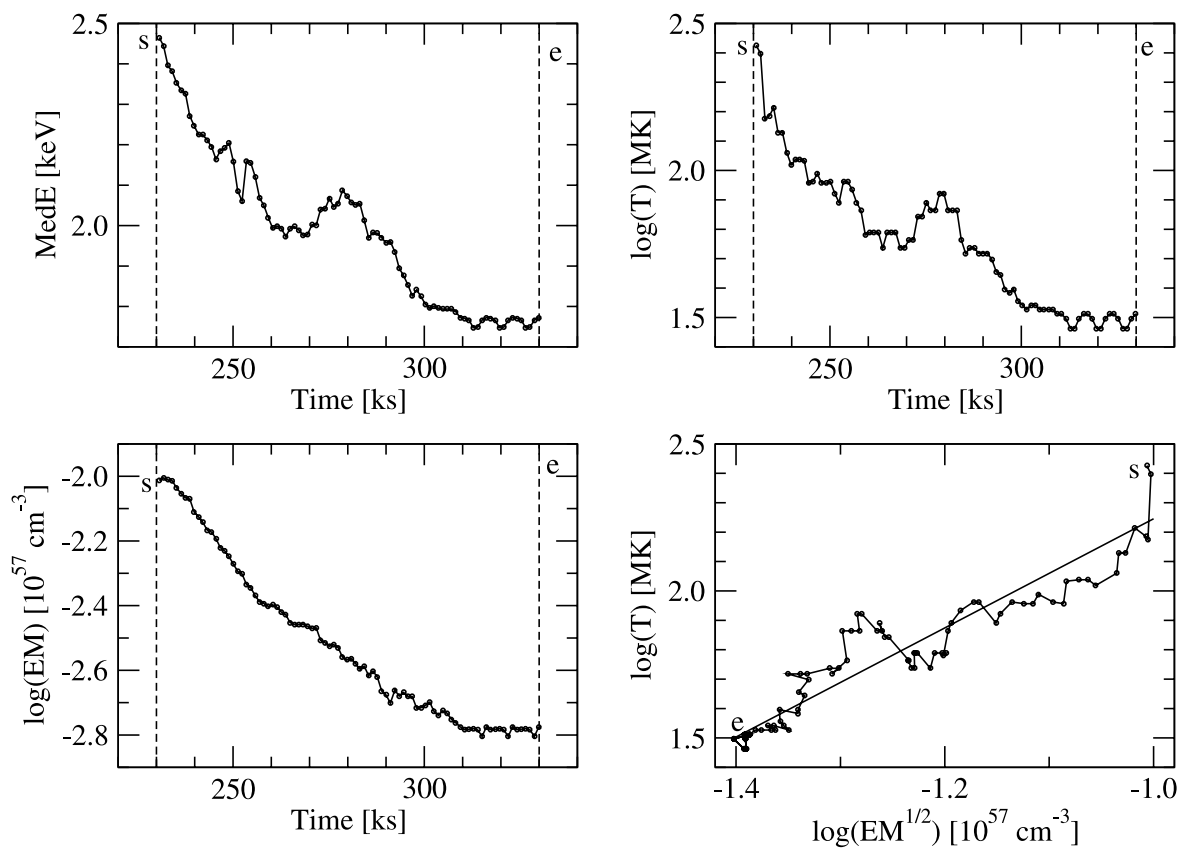

FIG. 30.-X-ray spectral modeling of the superflare in ONC COUP source 1343 using the method of the adaptively smoothed median energy with a window size of at

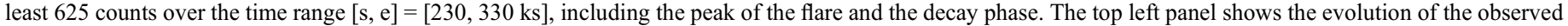

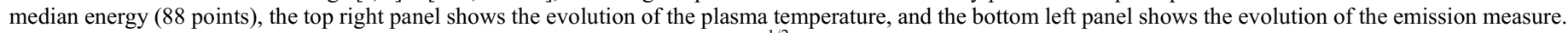
The bottom right panel shows the evolution of the flare in the $\log T-\log (\mathrm{EM})^{1 / 2}$ plane with the best-fitting decay as a solid line of slope $\zeta=1.86 \pm 0.29$. 

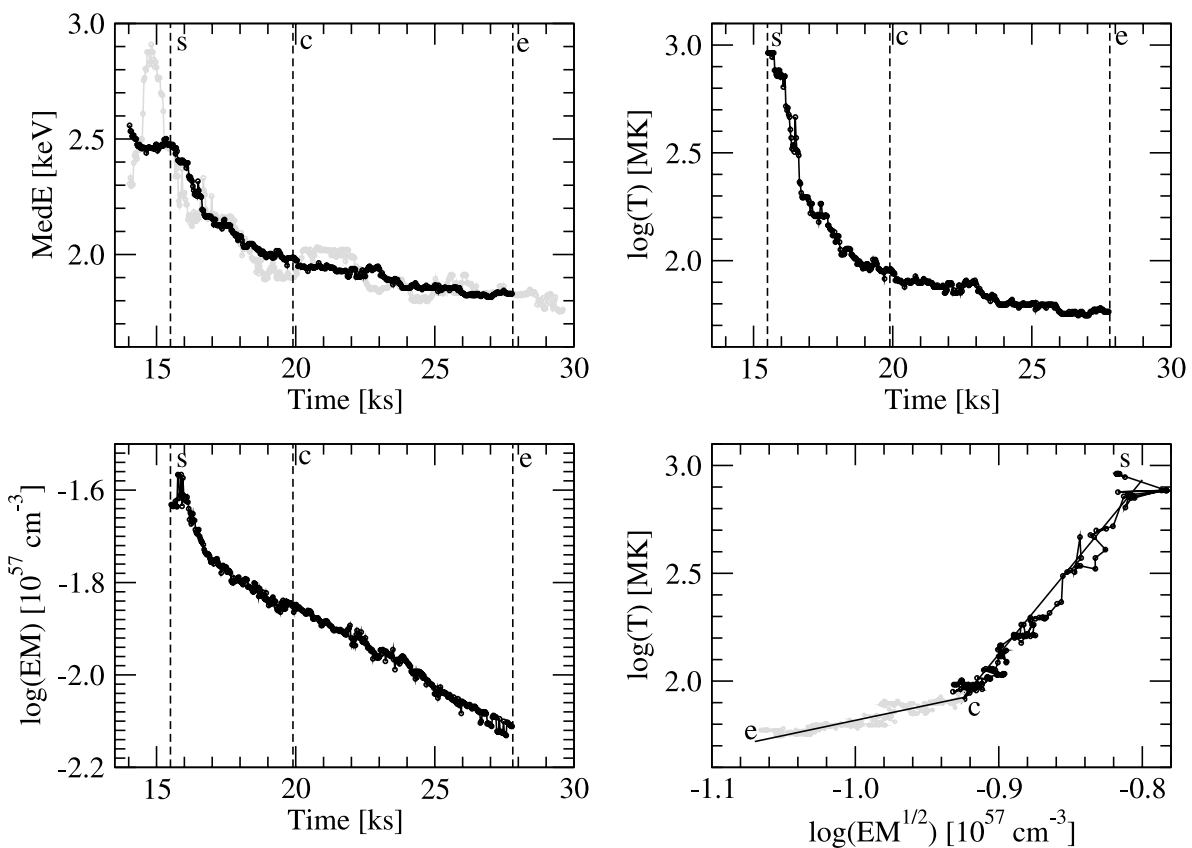

FIG. 31.-X-ray spectral modeling of the superflare in source 294 using the adaptively smoothed median energy with a window size of at least 570 counts over the time range $[\mathrm{s}, \mathrm{e}]=[15.5,27.8 \mathrm{ks}]$, including the decay phase of the flare (see Fig. 28). The top left panel shows the evolution of the smoothed median energy for the window sizes of 256 ( gray) and 570 (black) counts. The top right panel shows the evolution of the temperature (403 points); the bottom left panel shows the evolution of the emission measure. The bottom right panel shows the evolution of the flare in the $\log T-\log (\mathrm{EM})^{1 / 2}$ plane with the two best-fitting decays: a slope of $\zeta=8.08 \pm 0.96$ over the [s, c] $=[15.5,19.9 \mathrm{ks}]$ time range, and a slope of $\zeta=1.40 \pm 0.13$ over the [c, e] $=[19.9,27.8 \mathrm{ks}] \mathrm{range}$.

top left panel shows the trend of the median energy with 88 time points over the $100 \mathrm{ks}$ time range. The top right and bottom left panels show the derived decays of temperature and emission measure assuming a constant absorption of $\log N_{\mathrm{H}}=21.9 \mathrm{~cm}^{-2}$. Finally, the bottom right panel gives the $\log T-\log (\mathrm{EM})^{1 / 2} \mathrm{di}-$ agram over the $100 \mathrm{ks}$ peak-decay phase; the flare evolves from the upper right to the lower left. The best-fit decay slope is $\zeta=$ $1.86 \pm 0.29$. These last three panels look very similar to those shown in Figure 3 of Favata et al. (2005), who obtain a similar slope of $\zeta=1.95 \pm 0.51$ using coarser time sampling and XSPEC fits. This validates our use of adaptively smoothed median energies as estimates of plasma temperatures in flare loop models.

The method is applied to $12.3 \mathrm{ks}(15.5-27.8 \mathrm{ks}$ after the start of the observation) of the ACIS-I source 294 light curve; the results are shown in Figure 31. Trends of the median energy for two different smoothing kernels are alike within the studied decay phase of the flare. We report results for the 570 count kernel, although it does a poor job of tracing the earlier rapidly changing flare peak. The derived temperatures are very high, at least $T \sim$ $300 \mathrm{MK}$, and conceivably as high as $800-1000 \mathrm{MK}$ during the first $1 \mathrm{ks}$ of the decay phase. But they rapidly drop to $T \simeq 100 \mathrm{MK}$ over the next hour and then decrease more gradually to $T \simeq$ $60 \mathrm{MK}$ over the following $2 \mathrm{hr}$.

The derived $\log T-\log (\mathrm{EM})^{1 / 2}$ correlation (bottom right panel) suggests two decay phases: rapid cooling over the first $\sim 4.4 \mathrm{ks}$ with $\zeta=8.08 \pm 0.96$, followed by slow cooling with $\zeta=1.4 \pm$ 0.13 . The median temperatures in the top right panel are in good agreement with the cruder time-sliced spectroscopy shown in Figure $28 b$. The slow decay value $\zeta=1.4$ is close to the upper limit of 1.5 for a freely decaying loop with no prolonged heating input (Favata et al. 2005; Reale et al. 1997).

Since we observe only the first $\sim 4.4 \mathrm{hr}$ of the decay phase, we can obtain only a lower limit to the phase duration, $\tau_{\mathrm{lc}}>15 \mathrm{ks}$, and thus only a lower limit to the inferred loop length. Using for- mulae (5)-(7) in Favata et al. (2005) and truncating our peak temperature around $300 \mathrm{MK}$, we derive a loop length $L \gtrsim 6.4 \times$ $10^{11} \mathrm{~cm}$. We roughly estimate the stellar radius for source 294 to be $R_{\star} \sim 2.5 R_{\odot}$ using (Siess et al. 2000) PMS models for a $\sim 1$ Myr old star of $\sim 1.4 M_{\odot}$ mass (Table 2 ). The magnetic loop is then $\gtrsim 3.7$ times the radius of the star. This limit is even more conservative if the peak temperature exceeds $300 \mathrm{MK}$; the loop would be 2 times larger if a peak temperature around 800$1000 \mathrm{MK}$ were used, as suggested from the plasma temperatures derived in Figure 31. Source 294 may thus be another case where magnetic fields connect the star to the circumstellar disk, as discussed by Favata et al. (2005). Although there is no indication of the presence of $K$-band excess in source 294, it is well known that $J H K$ photometry alone is not sufficient to detect circumstellar material. Ground-based JHKLM or Spitzer Space Telescope photometry would allow much better discrimination between stars with and without circumstellar disks.

\section{CONCLUSIONS}

We present a $30 \mathrm{ks}$ (net exposure) Chandra ACIS observation of the Cepheus B molecular cloud and a portion of the nearby Cep OB3b OB association. The main conclusions of our study are as follows:

1. We detect $431 \mathrm{X}$-ray sources with a limiting luminosity for the region outside of the molecular cloud of $\sim 10^{29} \mathrm{ergs} \mathrm{s}^{-1} ; 384$ of them are unambiguously identified with 2MASS NIR sources. On visual inspection of 2MASS atlases we find NIR counterparts for at least 10 more soft and two more hard X-ray sources, which have not been registered in the 2MASS catalog. On the NIR colormagnitude diagram most of the detected X-ray sources occupy the locus of low-mass PMS stars with a typical absorption of $A_{V} \sim$ 2.5 mag (assuming an age of $\sim 1 \mathrm{Myr}$ ), but as many as 60 sources with NIR counterparts, residing close to/in the molecular cloud, have even higher absorptions, up to $A_{V} \sim 28 \mathrm{mag}$. 
2. Twenty-four unidentified hard X-ray sources are classified as possible extragalactic contaminants (Table 3, "a"). Although some of them still may be embedded members of the cloud, about $70 \%$ percent of them are probably true AGNs, as argued by a detailed simulation of the extragalactic background population, including instrumental background and local (Cepheus) plus large-scale Galactic absorptions. Through careful simulations of X-ray emission from the Galactic foreground and background populations employing a stellar population synthesis model of the Galactic disk, and through the inspection of NIR colors of X-raydetected sources, we expect to have no more than a few Galactic background stars, and we suggest 13 field star candidates (Table 3 , "f").

3. In order to obtain a reliable X-ray luminosity function of the detected PMS stars, we determine source X-ray fluxes based on knowledge of their NIR absorptions and X-ray median energies. We then perform careful MARX simulations to derive errors on median energy, combine them with known errors on count rate and absorption, and propagate them to the flux errors through Monte Carlo simulations.

4. We discover more than 380 PMS stars in the ACIS-I field of the Cepheus region, which provide the best census to date for the stellar population of the cloud. Based on $N_{\mathrm{H}}$ and $K$-band stratified spatial distributions of the detected PMS stars, we separate them into two rich clusters: the lightly obscured cluster (= "Cep OB3b within ACIS-I field") (321 stars; Table 3, "l"), and the embedded cluster (64 stars; Table 3, "e"). We estimate that another 400-450 (300-340) low-mass PMS stars are present in the unobscured (obscured) clusters within the Chandra field, but are too X-ray-faint to be individually identified.

5. We find that the XLF of the lightly obscured Cep OB3b cluster region within the ACIS-I field shows an excess of stars around $\log L_{\mathrm{X}} \sim 29.7 \mathrm{ergs} \mathrm{s}^{-1}(0.5-8 \mathrm{keV}$, after individual correction for absorption) compared to the well-studied Orion Nebula cluster. This may be caused by a nonstandard IMF in Cep OB3b with an excess of $\simeq 0.3 M_{\odot}$ stars if the cluster age is $\sim 1 \mathrm{Myr}$. This excess also appears in the independently derived KLF based on 2MASS source counts (after an uncertain subtraction of background counts from two control fields) that show more $K \sim$ 13-14 stars than expected from a standard IMF. However, the discrepancy may also be attributed to different ages and star formation histories for the Cep OB3b and ONC clusters.

6. We show that the O7n star HD 217086 with its known wind terminal velocity and mass-loss rate is unable to produce the bright soft X-ray diffuse emission seen in the field. An alternative explanation - that the X-ray diffuse emission originates from the unresolved PMS point sources in the region-is suggested by (1) the spatial coincidence of the diffuse emission with the detected point-source population, (2) the similarity of the plasma temperature of the diffuse emission to that of the combined weak PMS Orion-COUP sources, and (3) the equivalence of the amount of the observed X-ray luminosity of the diffuse emission with that of the estimated undetected members of the unobscured population.

7. We establish that the X-ray emission from HD 217086 is a constant soft-spectrum emission at the level $\log \left(L_{\mathrm{X}} / L_{\mathrm{bol}}\right) \sim-7$ that is expected from the standard model involving many small shocks in an unmagnetized radiatively accelerated wind.

8. From the evolution of an adaptively smoothed median energy during the unusually hot and strong flare in source 294, we infer the slope of the trajectory in the temperature-density diagram, estimate the lower limit on the size of the flaring structure, and suggest that source 294 may join a number of similar superflare sources seen in Orion clouds in which long magnetic structures may connect the protoplanetary disk to the stellar surface.

We thank the referee, Tim Naylor, for his time and many useful comments that improved this work. This work was supported by the ACIS team (G. Garmire, PI) through NASA contract NAS8-38252 and from Chandra contract SV4-74018 issued by the Chandra X-Ray Center operated by SAO under NASA contract NAS8-03060. M. T. acknowledges financial support from Japan Society for the Promotion of Science. This publication makes use of data products from the Two Micron All Sky Survey, which is a joint project of the University of Massachusetts and the Infrared Processing and Analysis Center/California Institute of Technology, funded by the National Aeronautics and Space Administration and the National Science Foundation.
Arnaud, K. A. 1996, in ASP Conf. Ser. 101, Astronomical Data Analysis Software and Systems V, ed. G. H. Jacoby \& J. Barnes (San Francisco: ASP), 17 Babel, J., \& Montmerle, T. 1997, ApJ, 485, L29

Baraffe, I., Chabrier, G., Allard, F., \& Hauschildt, P. H. 1998, A\&A, 337, 403 Blaauw, A. 1964, ARA\&A, 2, 213

Blaauw, A., Hiltner, W. A., \& Johnson, H. L. 1959, ApJ, 130, 69

Brandt, W. N., et al. 2001, AJ, 122, 2810

Burningham, B., Naylor, T., Littlefair, S. P., \& Jeffries, R. D. 2005, MNRAS, 363, 1389

Corbelli, E., Palla, F., \& Zinnecker, H. 2005, The Initial Mass Function 50 Years Later, ed. E. Corbelli, F. Palle, \& H. Zinnecker (Dordrecht: Springer) Cox, A. N. 2000, Allen's Astrophysical Quantities (4th ed.; New York: Springer) Crawford, D. L., \& Barnes, J. V. 1970, AJ, 75, 952

Dickey, J. M., \& Lockman, F. J. 1990, ARA\&A, 28, 215

Elmegreen, B. G. 1999, ApJ, 515, 323

2004, MNRAS, 354, 367

Elmegreen, B. G., \& Lada, C. J. 1977, ApJ, 214, 725

Favata, F., Flaccomio, E., Reale, F., Micela, G., Sciortino, S., Shang, H., Stassun, K. G., \& Feigelson, E. D. 2005, ApJS, 160, 469

Feigelson, E. D., Broos, P., Gaffney, J. A., Garmire, G., Hillenbrand, L. A., Pravdo, S. H., Townsley, L., \& Tsuboi, Y. 2002, ApJ, 574, 258

Feigelson, E. D., \& Getman, K. V. 2005, in The Initial Mass Function 50 Years

Later, ed. E. Corbelli, F. Palle, \& H. Zinnecker (Dordrecht: Springer), 163

Feigelson, E. D., et al. 2004, ApJ, 611, 1107 2005, ApJS, 160, 379

Felli, M., Tofani, G., Harten, R. H., \& Panagia, N. 1978, A\&A, 69, 199
Garmire, G. P., Bautz, M. W., Ford, P. G., Nousek, J. A., \& Ricker, G. R. 2003, Proc. SPIE, 4851, 28

Getman, K. V., Feigelson, E. D., Grosso, N., McCaughrean, M. J., Micela, G.,

Broos, P., Garmire, G., \& Townsley, L. 2005a, ApJS, 160, 353

Getman, K. V., et al. 2005b, ApJS, 160, 319

Grosso, N., Montmerle, T., Feigelson, E. D., \& Forbes, T. G. 2004, A\&A, 419, 653

Grosso, N., et al. 2005, ApJS, 160, 530

Güdel, M., Guinan, E. F., \& Skinner, S. L. 1998, in ASP Conf. Ser. 154, Cool Stars, Stellar Systems, and the Sun, ed. R. A. Donahue \& J. A. Bookbinder (San Francisco: ASP), 1041

Howarth, I. D., \& Prinja, R. K. 1989, ApJS, 69, 527

Hünsch, M., Schmitt, J. H. M. M., Sterzik, M. F., \& Voges, W. 1999, A\&AS, 135,319

Imanishi, K., Koyama, K., \& Tsuboi, Y. 2001, ApJ, 557, 747

Jordi, C., Trullols, E., \& Galadi-Enriquez, D. 1996, A\&A, 312, 499

Kroupa, P. 2002, Science, 295, 82

Lada, E. A., \& Lada, C. J. 1995, AJ, 109, 1682

Lucy, L. B., \& White, R. L. 1980, ApJ, 241, 300

Minchin, N. R., Ward-Thompson, D., \& White, G. J. 1992, A\&A, 265, 733

Moreno-Corral, M. A., Chavarria, K. C., de Lara, E., \& Wagner, S. 1993, A\&A, 273, 619

Moretti, A., Campana, S., Lazzati, D., \& Tagliaferri, G. 2003, ApJ, 588, 696

Muench, A. A., Lada, E. A., Lada, C. J., \& Alves, J. 2002, ApJ, 573, 366

Naylor, T., \& Fabian, A. C. 1999, MNRAS, 302, 714

Panagia, N., \& Thum, C. 1981, A\&A, 98, 295 
Pizzolato, N., Maggio, A., \& Sciortino, S. 2000, A\&A, 361, 614

Pozzo, M., Naylor, T., Jeffries, R. D., \& Drew, J. E. 2003, MNRAS, 341, 805

Preibisch, T., \& Feigelson, E. D. 2005, ApJS, 160, 390

Preibisch, T., et al. 2005, ApJS, 160, 401

Prinja, R. K., Barlow, M. J., \& Howarth, I. D. 1990, ApJ, 361, 607

Reale, F., Betta, R., Peres, G., Serio, S., \& McTiernan, J. 1997, A\&A, 325, 782

Robin, A. C., Reylé, C., Derrière, S., \& Picaud, S. 2003, A\&A, 409, 523

Ryter, C. E. 1996, Ap\&SS, 236, 285

Sargent, A. I. 1977 , ApJ, 218, 736 1979, ApJ, 233, 163

Schmitt, J. H. M. M. 1997, A\&A, 318, 215

Schmitt, J. H. M. M., Fleming, T. A., \& Giampapa, M. S. 1995, ApJ, 450, 392

Siess, L., Dufour, E., \& Forestini, M. 2000, A\&A, 358, 593

Smith, R. K., Brickhouse, N. S., Liedahl, D. A., \& Raymond, J. C. 2001, ApJ, 556, L91
Stelzer, B., Flaccomio, E., Montmerle, T., Micela, G., Sciortino, S., Favata, F., Preibisch, T., \& Feigelson, E. D. 2005, ApJS, 160, 557

Stevens, I. R., \& Hartwell, J. M. 2003, MNRAS, 339, 280

Testi, L., Olmi, L., Hunt, L., Tofani, G., Felli, M., \& Goldsmith, P. 1995, A\&A, 303, 881

Townsley, L. K., Broos, P. S., Nousek, J. A., \& Garmire, G. P. 2002, Nucl. Instrum. Methods Phys. Res. A, 486, 751

Townsley, L. K., Feigelson, E. D., Montmerle, T., Broos, P. S., Chu, Y.-H., \& Garmire, G. P. 2003, ApJ, 593, 874

Tsuboi, Y., Koyama, K., Murakami, H., Hayashi, M., Skinner, S., \& Ueno, S. 1998, ApJ, 503, 894

ud-Doula, A., \& Owocki, S. P. 2002, ApJ, 576, 413

Weisskopf, M. C., Brinkman, B., Canizares, C., Garmire, G., Murray, S., \& Van Speybroeck, L. P. 2002, PASP, 114, 1

Wolk, S. J., Harnden, F. R., Flaccomio, E., Micela, G., Favata, F., Shang, H., \& Feigelson, E. D. 2005, ApJS, 160, 423 